\title{
Silver-Catalyzed Enantioselective Mannich Reaction of Diazoacetate Esters with $N$-Boc Aldimines
}

\author{
Gerard P. Robertson, Alistair J. M. Farley and Darren J. Dixon* \\ Department of Chemistry, Chemistry Research Laboratory,
}

University of Oxford, 12 Mansfield Road,

Oxford, OX1 3TA, UK

1 Supplementary Optimization Tables ...........................................................................S4

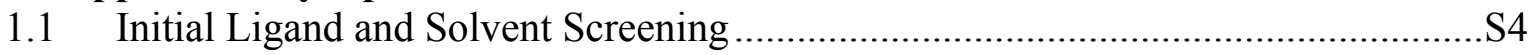

1.2 Further Ligand and Solvent Screening ...................................................................... 5

1.3 Optimisation of remaining parameters with $(R)$-DM-SEGPHOS ..............................S6

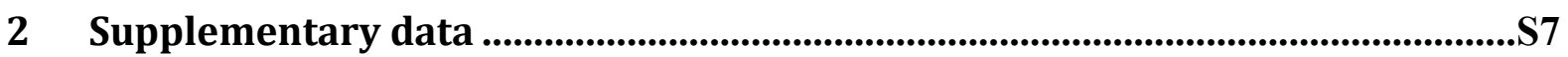

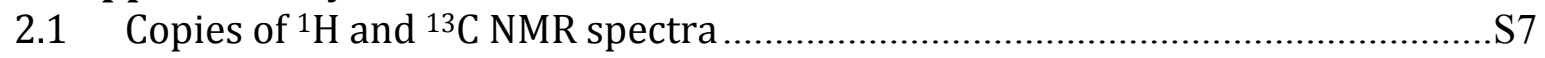

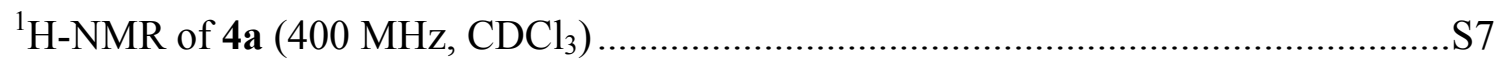

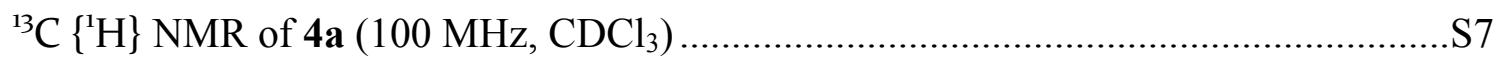

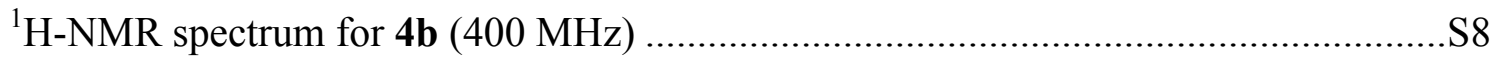

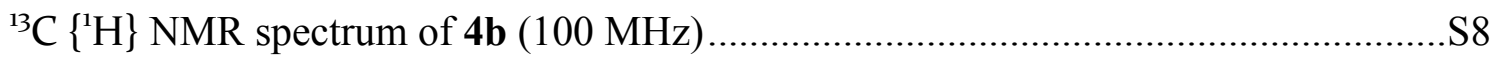

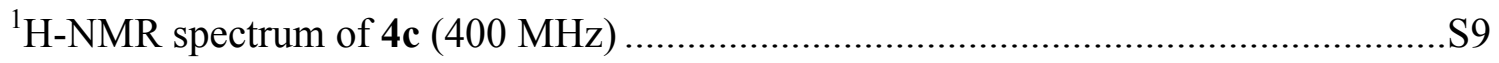

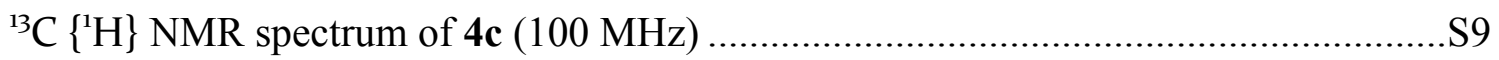

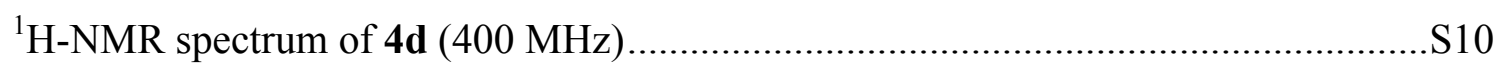

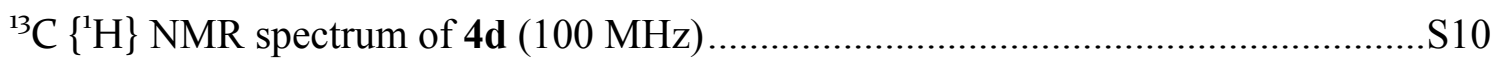

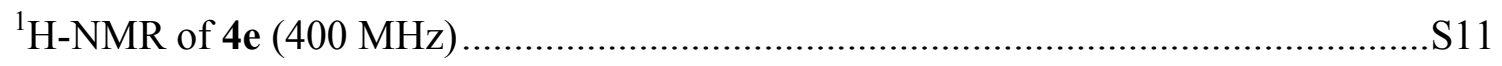

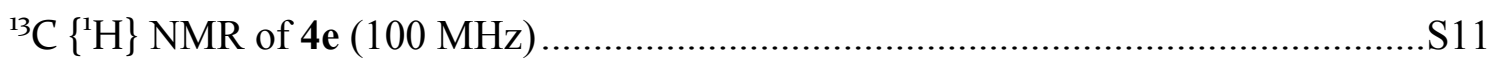

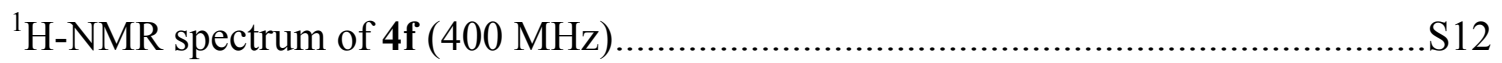

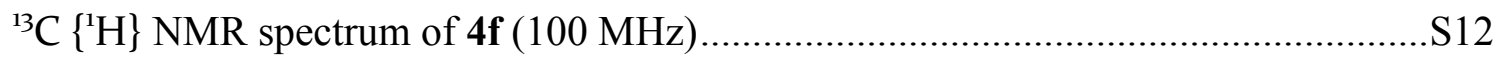

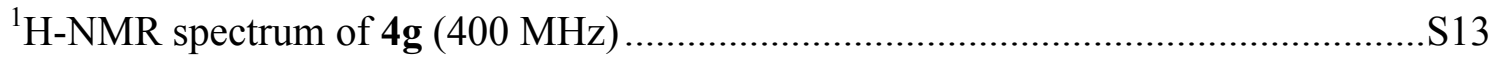

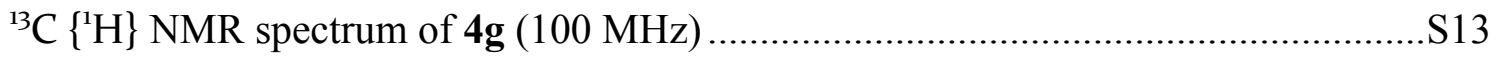

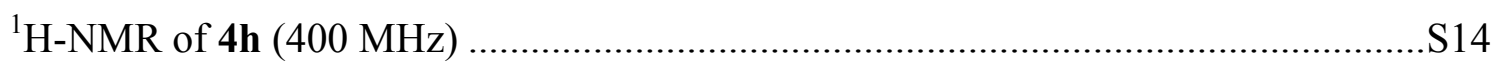

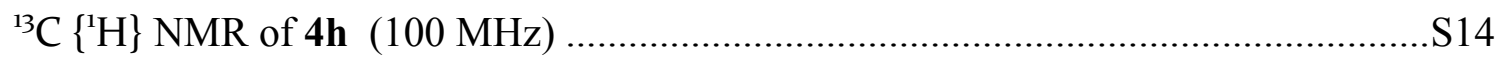

${ }^{1} \mathrm{H}-\mathrm{NMR}$ spectrum of $\mathbf{4 i}(400 \mathrm{MHz})$............................................................................. 


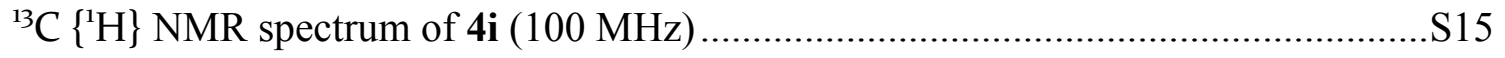

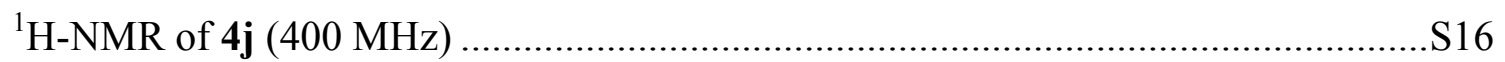

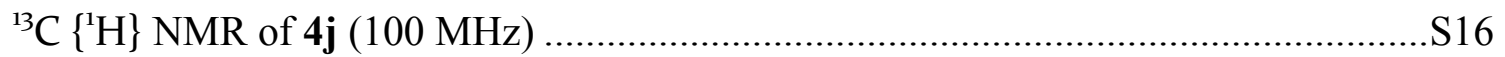

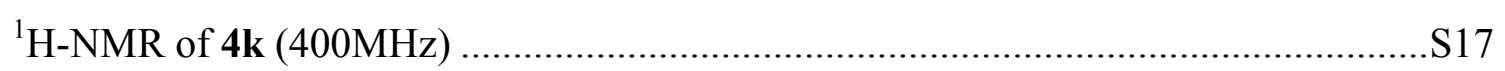

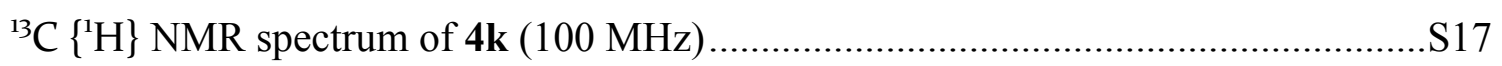

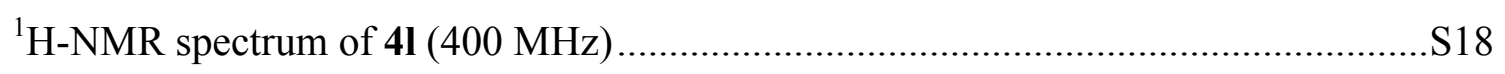

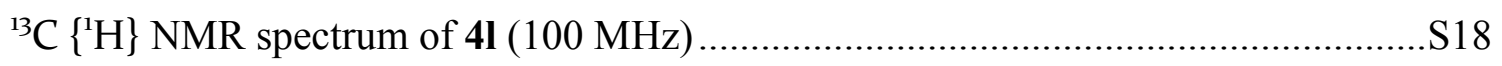

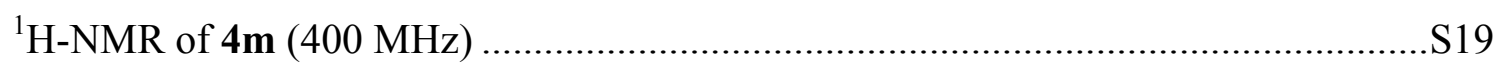

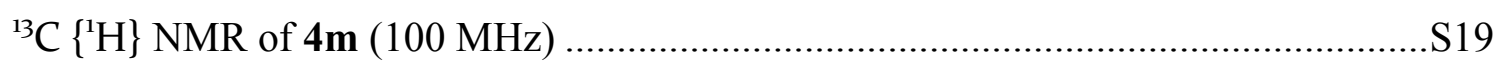

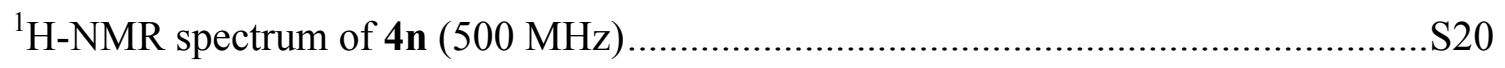

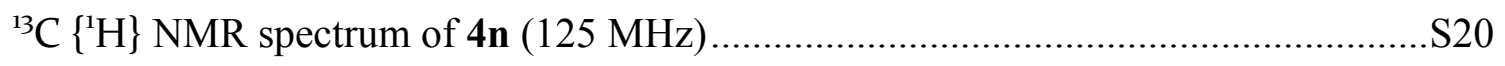

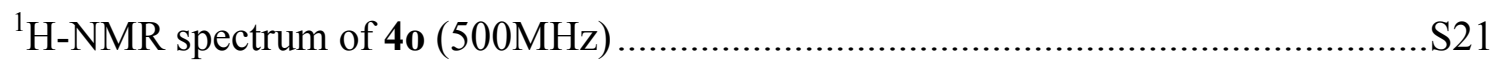

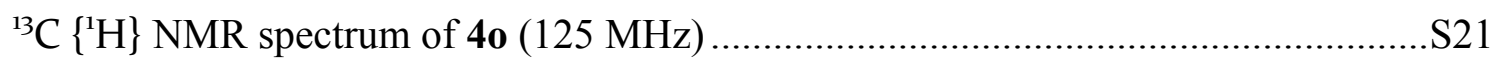

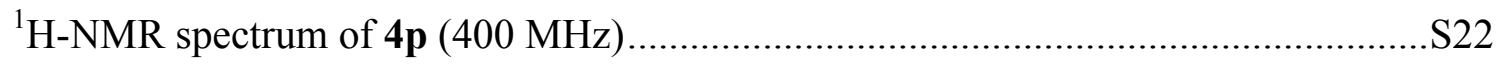

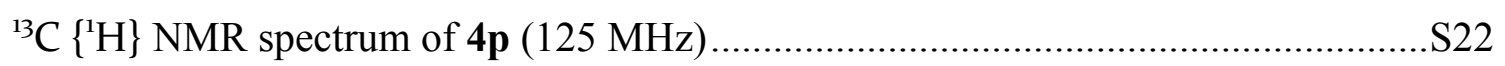

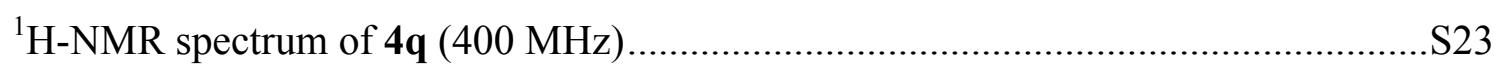

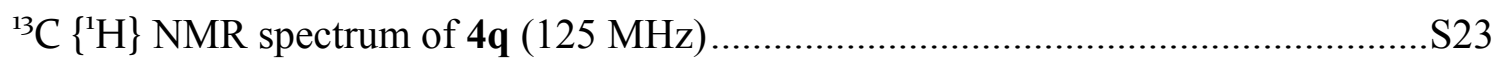

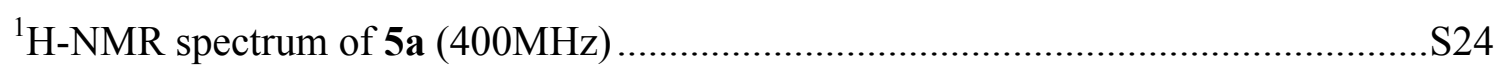

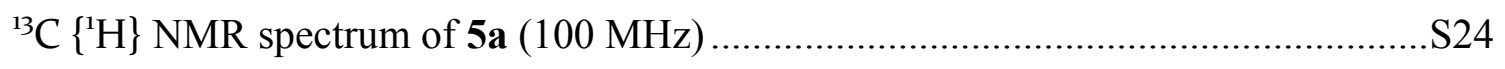

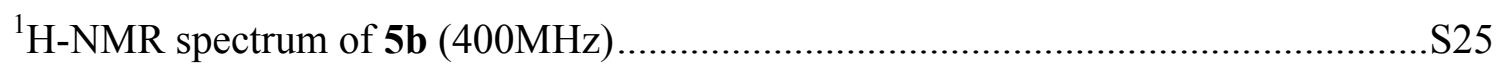

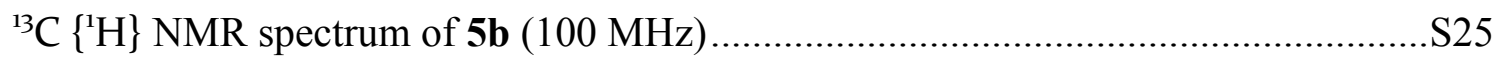

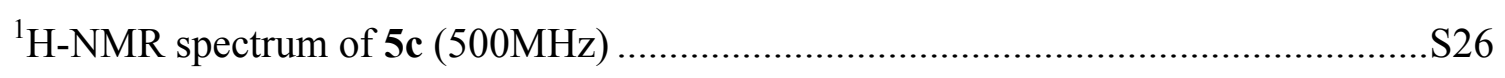

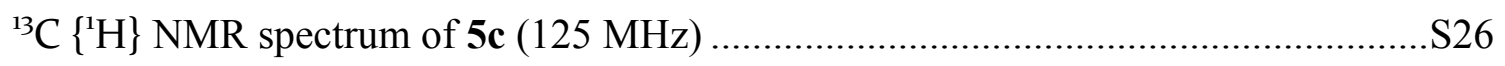

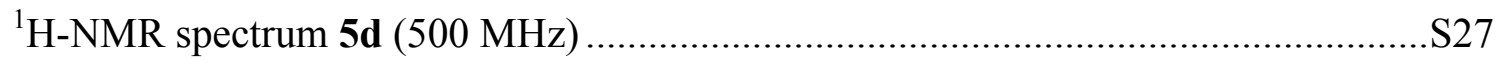

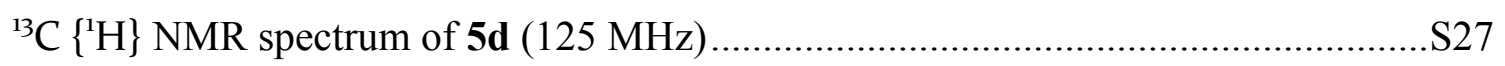

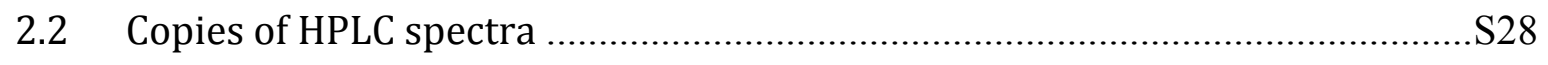

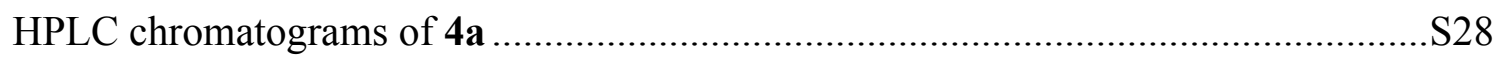

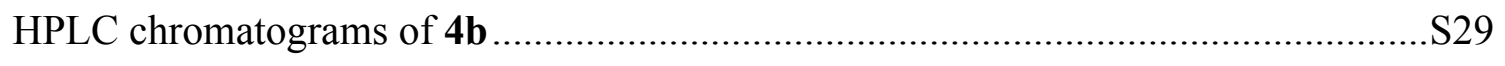

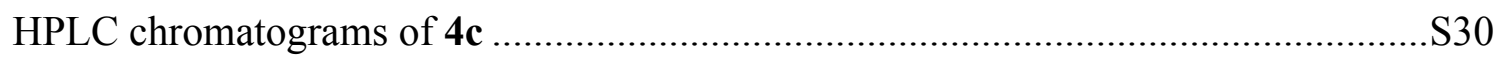

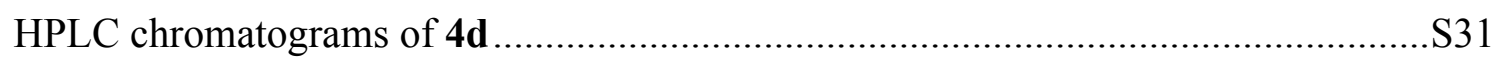

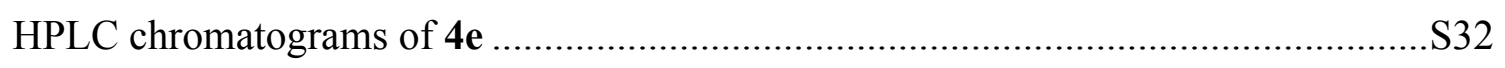

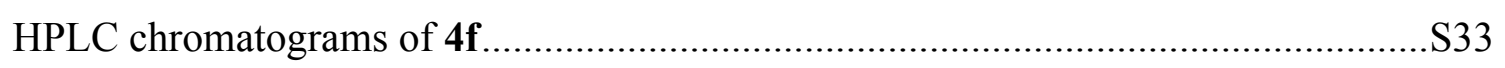

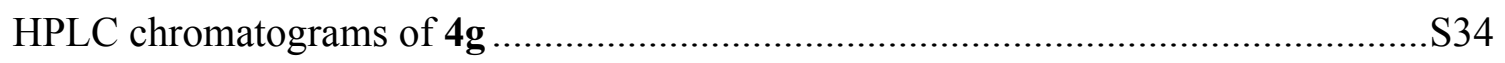

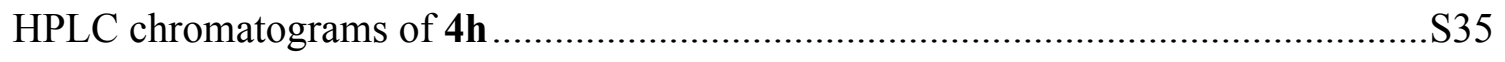

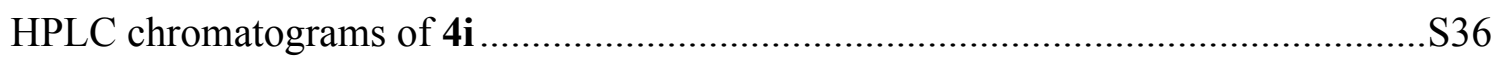




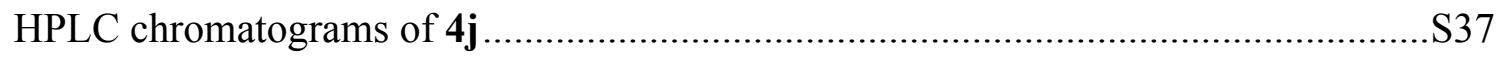

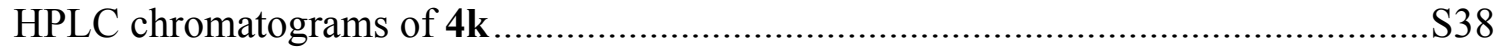

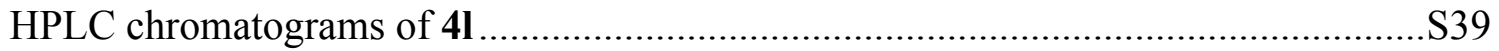

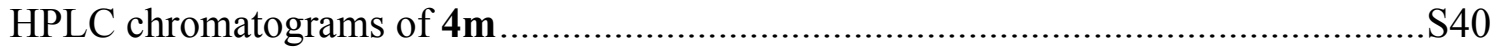

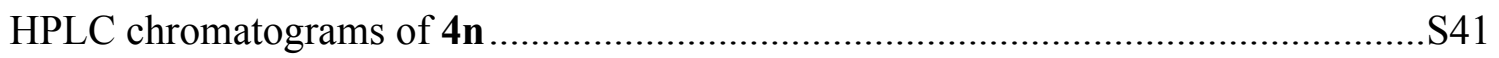

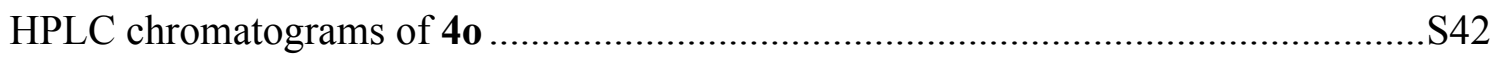

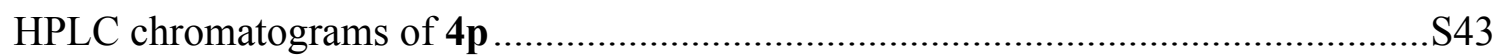

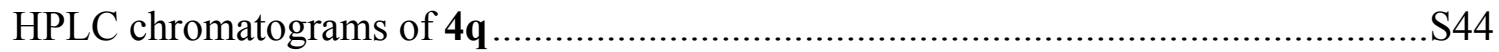

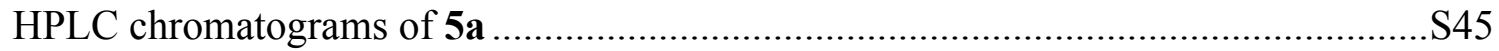

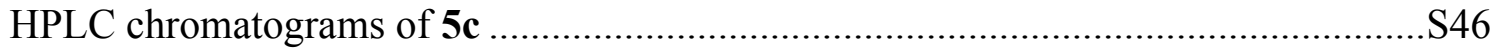

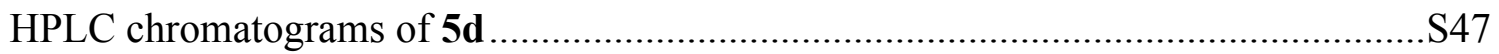




\section{Supplementary Optimization Tables}

\subsection{Initial Ligand and Solvent Screening}

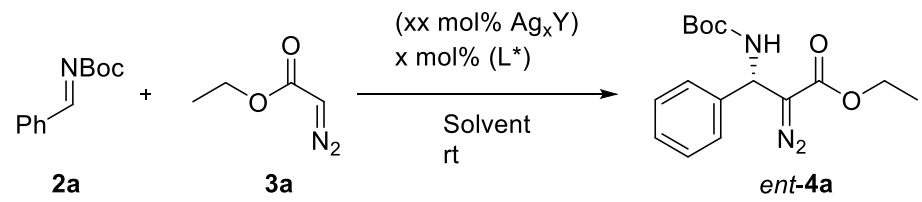

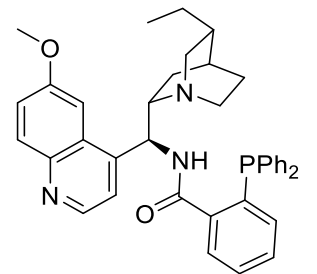

A

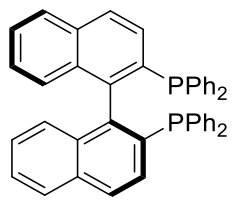

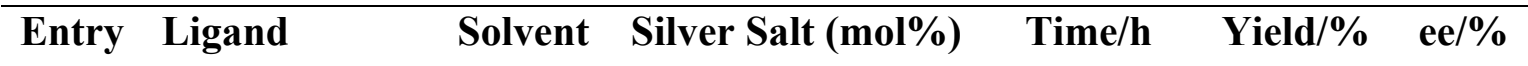

(mol\%)

\begin{tabular}{|c|c|c|c|c|c|c|}
\hline 1 & $\mathbf{A}(10 \mathrm{~mol} \%)$ & EtOAc & $\mathrm{Ag}_{2} \mathrm{O}(10 \mathrm{~mol} \%)$ & 15 & 81 & 9 \\
\hline 2 & $\mathbf{A}(20 \mathrm{~mol} \%)$ & EtOAc & $\mathrm{Ag}_{2} \mathrm{O}(5 \mathrm{~mol} \%)$ & 24 & 32 & 9 \\
\hline 3 & $\mathbf{A}(10 \mathrm{~mol} \%)$ & $\mathrm{Et}_{2} \mathrm{O}$ & $\mathrm{Ag}_{2} \mathrm{O}(10 \mathrm{~mol} \%)$ & 24 & 58 & 0 \\
\hline 4 & A $(10 \mathrm{~mol} \%)$ & Toluene & $\mathrm{Ag}_{2} \mathrm{O}(10 \mathrm{~mol} \%)$ & 44 & 13 & 9 \\
\hline 5 & B (10 mol\%) & EtOAc & $\mathrm{Ag}_{2} \mathrm{O}(10 \mathrm{~mol} \%)$ & 5 & 87 & 24 \\
\hline 6 & B (10 mol\%) & EtOAc & AgOTf (10 mol\%) & 4 & 74 & 37 \\
\hline 7 & B (10 mol\%) & EtOAc & $\operatorname{AgOTf}(5 \mathrm{~mol} \%)$ & 4 & 71 & 39 \\
\hline 8 & B $(10 \mathrm{~mol} \%)$ & EtOAc & $\operatorname{AgOTf}(20 \mathrm{~mol} \%)$ & 4 & 52 & 35 \\
\hline 9 & B (10 mol\%) & $\mathrm{CH}_{2} \mathrm{Cl}_{2}$ & $\operatorname{AgOTf}(5 \mathrm{~mol} \%)$ & 6 & 38 & 49 \\
\hline 10 & B (10 mol\%) & $\mathrm{Et}_{2} \mathrm{O}$ & $\operatorname{AgOTf}(5 \mathrm{~mol} \%)$ & 6 & 28 & 21 \\
\hline 11 & B (10 mol\%) & $\mathrm{CH}_{2} \mathrm{Cl}_{2}$ & AgOTf (5 mol\%) & 4 & 50 & $49^{[a]}$ \\
\hline
\end{tabular}

Optimisation Table S1: All reactions carried out on a $0.10 \mathrm{mmol}$ scale, $0.08 \mathrm{M}$ concentration wrt 2a. ${ }^{[a]}$ Reaction with $10 \mathrm{~mol}^{\%} \mathrm{NEt}_{3}$ as additive. 


\subsection{Further Ligand and Solvent Screening}

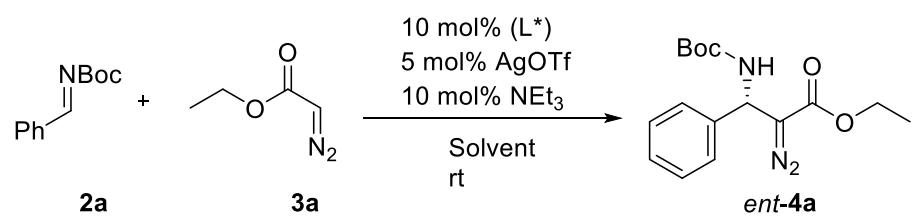

Phosphoramidite

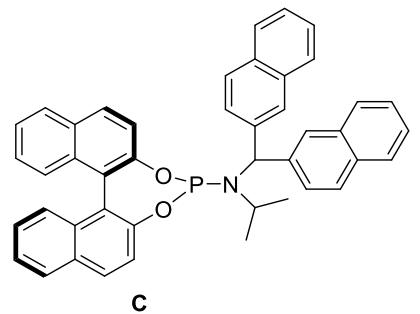

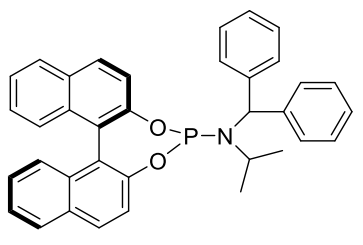

D<smiles>CCCc1c2ccc(OPN([C@H](C)c3ccccc3)[C@H](C)c3ccccc3)c1-c1c(ccc3c1CCCC3)CCCC2</smiles>

E

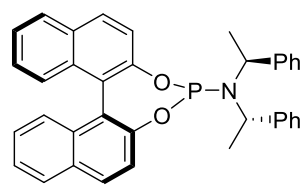

H<smiles>COc1cccc(-c2ccccc2)c1-c1c(OC)cccc1-c1ccccc1</smiles>

SEGPHOS
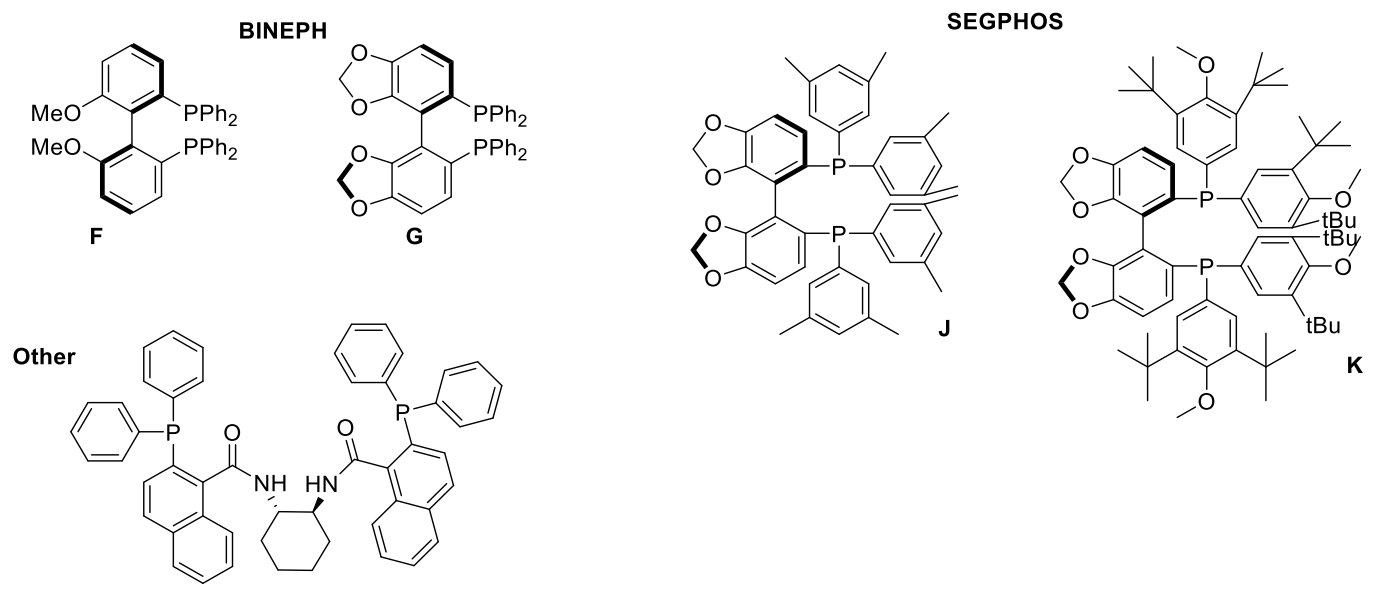

\begin{tabular}{llllll} 
Entry & Ligand (mol\%) & Solvent & Time/h & Yield/\% & ee/\% \\
\hline 1 & $\mathbf{C}(10 \mathrm{~mol} \%)$ & $\mathrm{EtOAc}$ & 16 & 74 & $-7^{[\mathrm{b}]}$ \\
2 & $\mathbf{D}(10 \mathrm{~mol} \%)$ & $\mathrm{CH}_{2} \mathrm{Cl}_{2}$ & 5 & 68 & 9 \\
3 & $\mathbf{E}$ & $\mathrm{CH}_{2} \mathrm{Cl}_{2}$ & 5 & 74 & 15 \\
4 & $\mathbf{F}$ & $\mathrm{CH}_{2} \mathrm{Cl}_{2}$ & 2 & 68 & $-51^{[\mathrm{b}]}$ \\
5 & $\mathbf{G}$ & $\mathrm{CH}_{2} \mathrm{Cl}_{2}$ & 5 & 52 & $-59^{[\mathrm{b}]}$ \\
6 & $\mathbf{H}$ & $\mathrm{EtOAc}^{2}$ & 7 & 65 & $-33^{[\mathrm{b}]}$ \\
7 & $\mathbf{I}$ & $\mathrm{CH}_{2} \mathrm{Cl}_{2}$ & 48 & 0 & - \\
$\mathbf{8}$ & $\mathbf{J}$ & $\mathrm{CH}_{2} \mathrm{Cl}_{2}$ & $\mathbf{5}$ & $\mathbf{5 8}$ & $\mathbf{- 8 1 ^ { [ \mathbf { b } ] }}$ \\
9 & $\mathbf{K}$ & $\mathrm{CH}_{2} \mathrm{Cl}_{2}$ & 6.5 & 58 & $-71^{[\mathrm{b}]}$
\end{tabular}

Optimisation Table S2: All reactions carried out on a $0.10 \mathrm{mmol} \mathrm{scale,} 0.08 \mathrm{M}$ concentration. All ees are referenced against the ee produced with $(S)$-BINAP as a ligand. ${ }^{[b]}$ Opposite enantiomer obtained. 


\subsection{Optimisation of remaining parameters with $(R)$-DM-SEGPHOS}
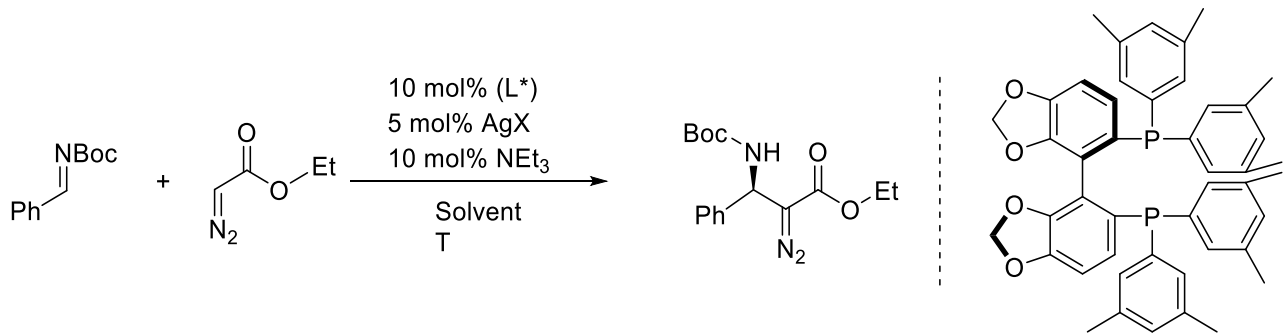

\begin{tabular}{|c|c|c|c|c|c|c|c|}
\hline Entry & Temperature $/{ }^{\circ} \mathrm{C}$ & Conc/mM & AgX & Solvent & Time/h & Yield/\% & ee/\% \\
\hline 1 & RT & 0.08 & AgOTf & $\mathrm{ClPh}$ & 6 & 65 & 75 \\
\hline 2 & RT & 0.08 & AgOTf & $\mathrm{CHCl}_{3}$ & 6 & 48 & 85 \\
\hline 3 & RT & 0.08 & $\mathrm{AgSbF}_{6}$ & $\mathrm{CHCl}_{3}$ & 72 & 16 & 69 \\
\hline 4 & -20 & 0.08 & AgOTf & $\mathrm{CHCl}_{3}$ & 24 & 32 & 91 \\
\hline 5 & $\mathrm{RT}$ & 0.08 & AgOTf & $\mathrm{CCl}_{4}$ & 6 & 77 & 25 \\
\hline 6 & -20 & 0.08 & $\mathrm{AgOAc}$ & $\mathrm{CHCl}_{3}$ & 16 & 32 & $91^{[\mathrm{c}]}$ \\
\hline 7 & -20 & 0.16 & AgOTf & $\mathrm{CHCl}_{3}$ & 16 & 68 & 88 \\
\hline 8 & -40 & 0.16 & AgOTf & $\mathrm{CHCl}_{3}$ & 24 & 77 & 97 \\
\hline 9 & -50 & 0.16 & AgOTf & $\mathrm{CHCl}_{3}$ & 24 & 68 & 97 \\
\hline 10 & -40 & 0.08 & AgOTf & $\mathrm{CHCl}_{3}$ & 72 & 40 & 91 \\
\hline 11 & -40 & 0.12 & AgOTf & $\mathrm{CHCl}_{3}$ & 24 & 40 & 95 \\
\hline 12 & -40 & 0.20 & AgOTf & $\mathrm{CHCl}_{3}$ & 17 & 70 & 97 \\
\hline
\end{tabular}

Optimisation Table S3. ${ }^{[c]}$ Reaction performed in the absence of $\mathrm{NEt}_{3}$. 
2 Supplementary data

2.1 Copies of ${ }^{1} \mathrm{H}$ and ${ }^{13} \mathrm{C}$ NMR spectra

${ }^{1} \mathrm{H}-\mathrm{NMR}$ of $4 \mathbf{a}\left(400 \mathrm{MHz}, \mathrm{CDCl}_{3}\right)$

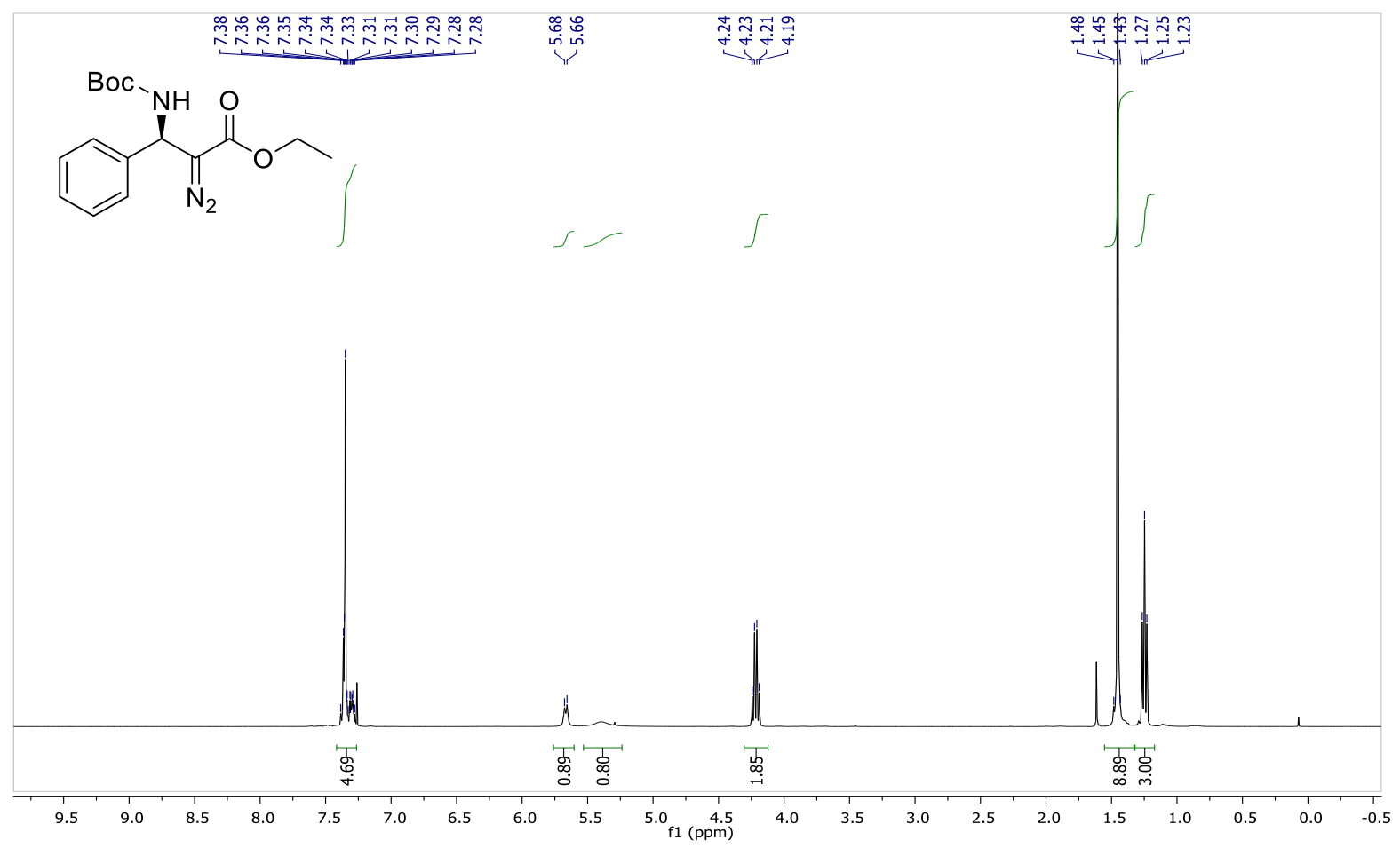

${ }^{13} \mathrm{C}\left\{{ }^{1} \mathrm{H}\right\}$ NMR of $4 \mathbf{a}\left(100 \mathrm{MHz}, \mathrm{CDCl}_{3}\right)$

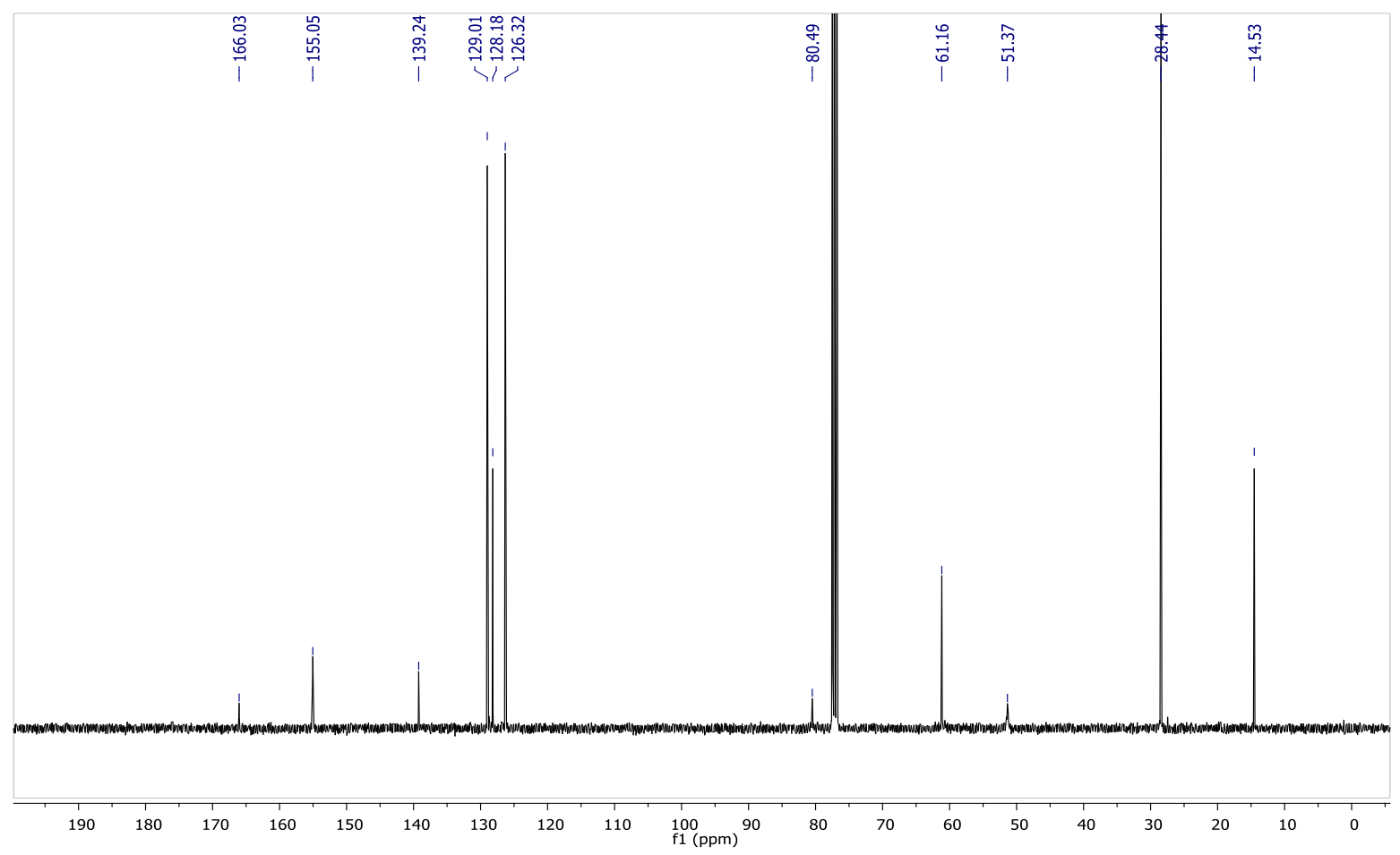

ST 
${ }^{1} \mathrm{H}-\mathrm{NMR}$ spectrum for $\mathbf{4 b}(400 \mathrm{MHz})$

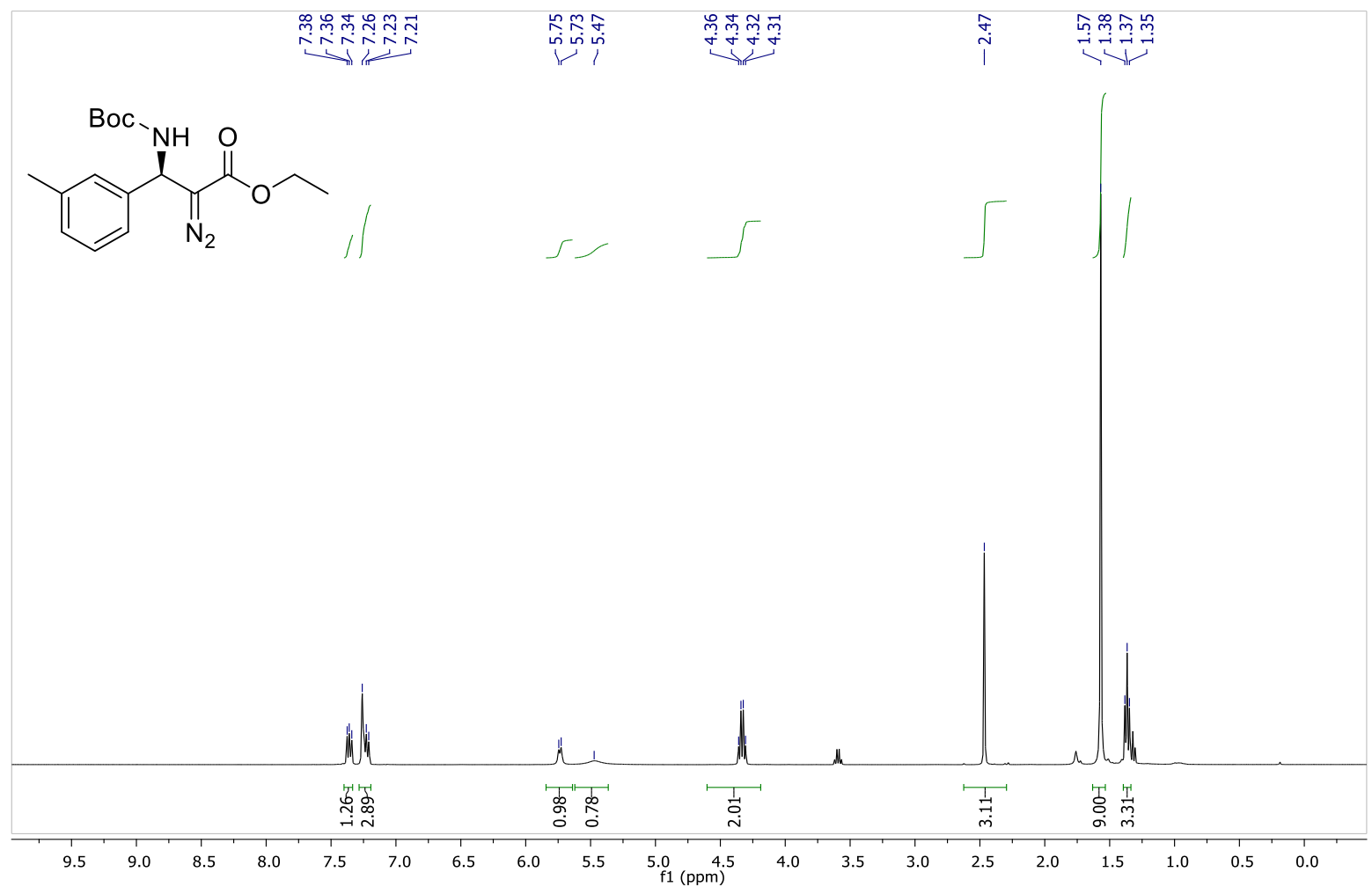

${ }^{13} \mathrm{C}\left\{{ }^{1} \mathrm{H}\right\}$ NMR spectrum of $\mathbf{4 b}(100 \mathrm{MHz})$

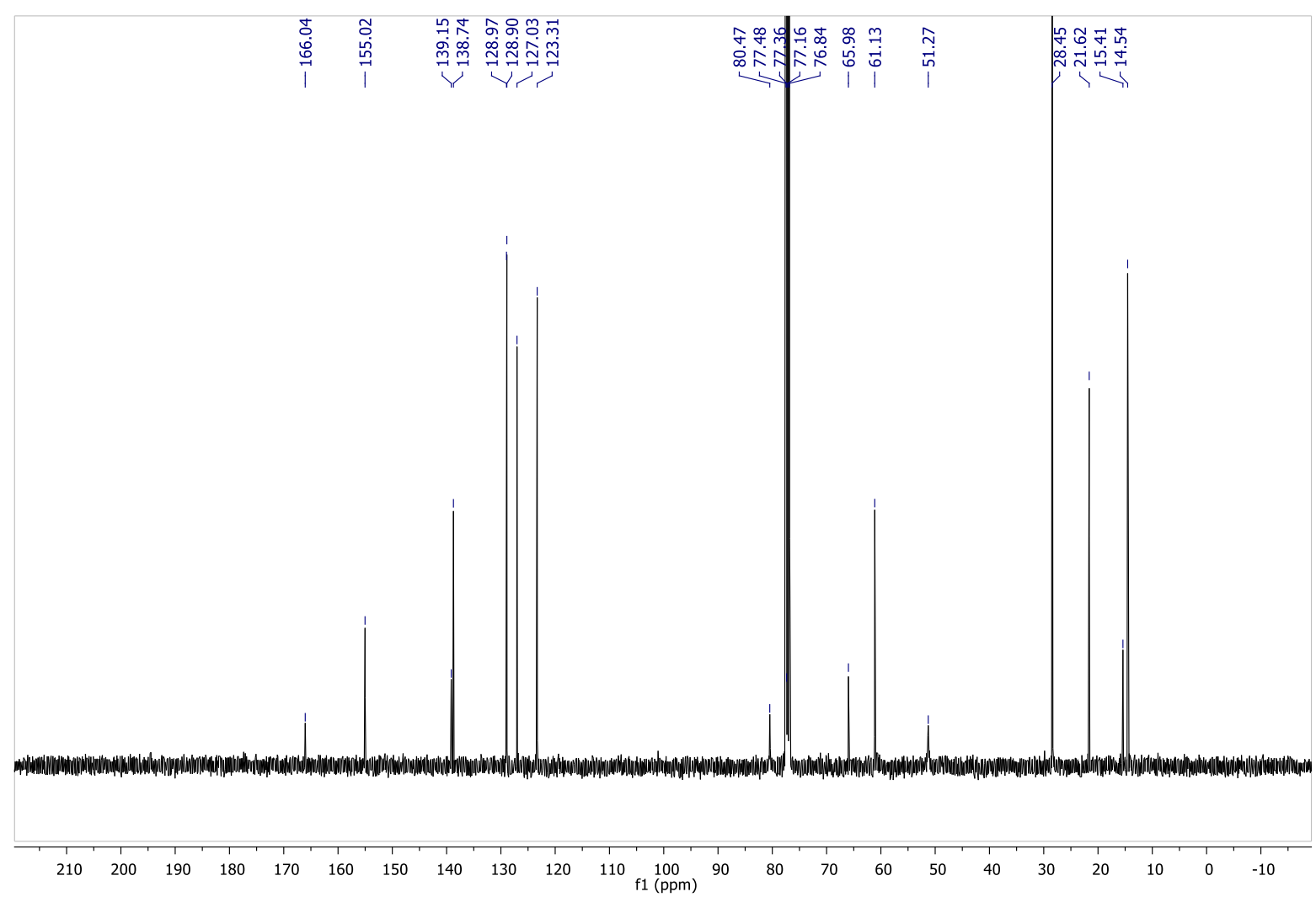


${ }^{1} \mathrm{H}-\mathrm{NMR}$ spectrum of $\mathbf{4 c}(400 \mathrm{MHz})$

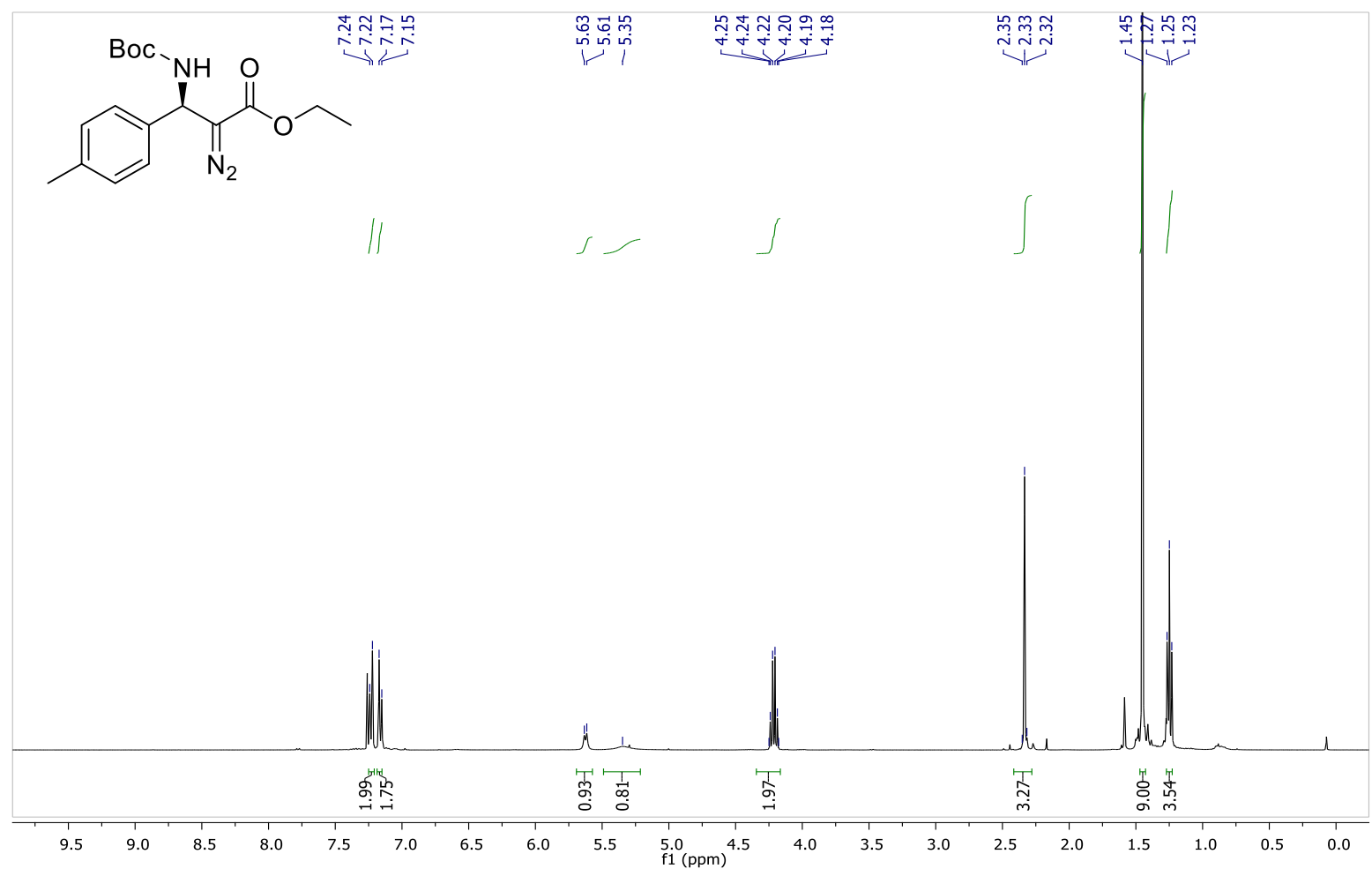

${ }^{13} \mathrm{C}\left\{{ }^{1} \mathrm{H}\right\}$ NMR spectrum of $4 \mathbf{c}(100 \mathrm{MHz})$

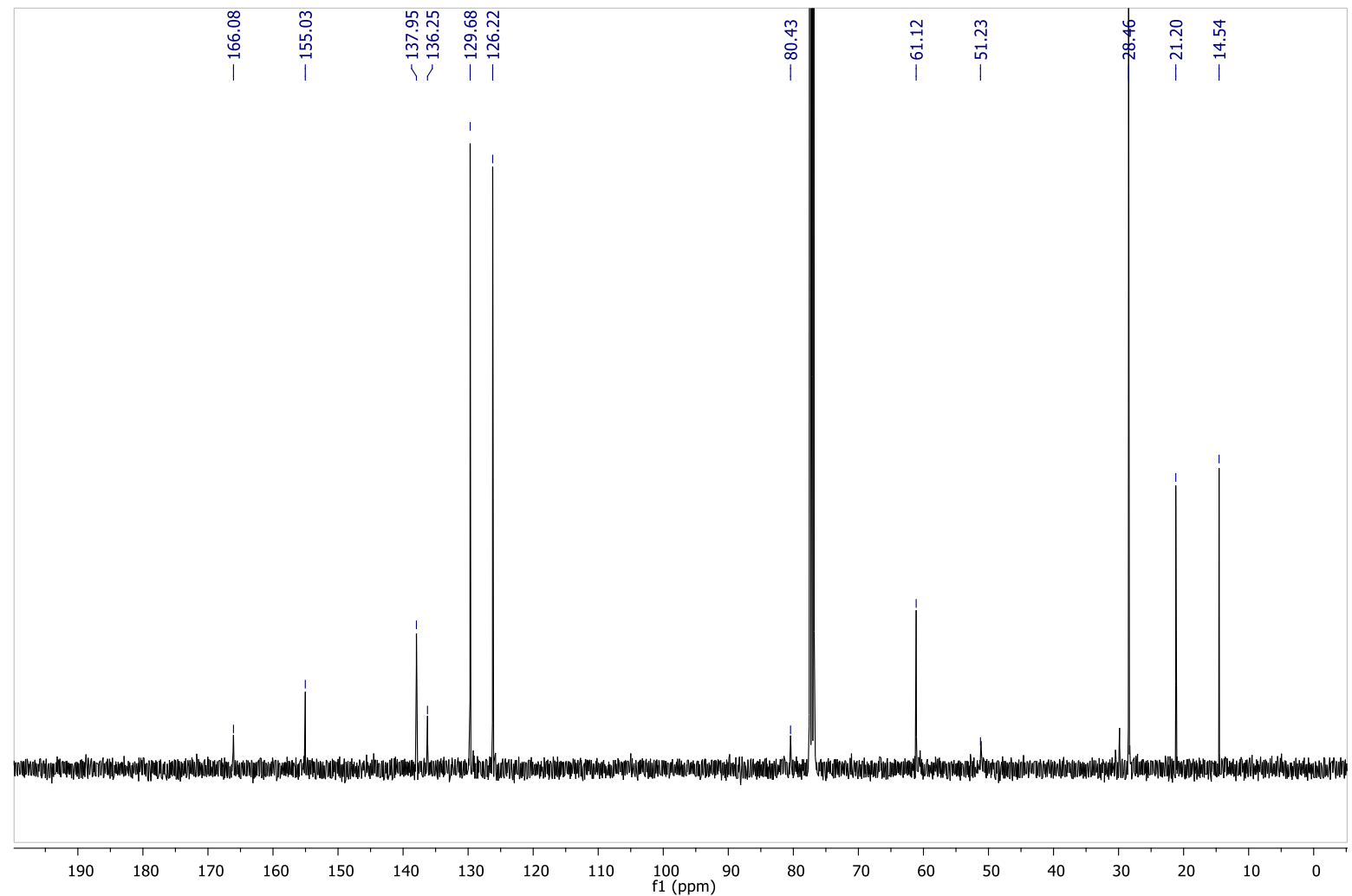


${ }^{1} \mathrm{H}-\mathrm{NMR}$ spectrum of $\mathbf{4 d}$ (400 MHz)

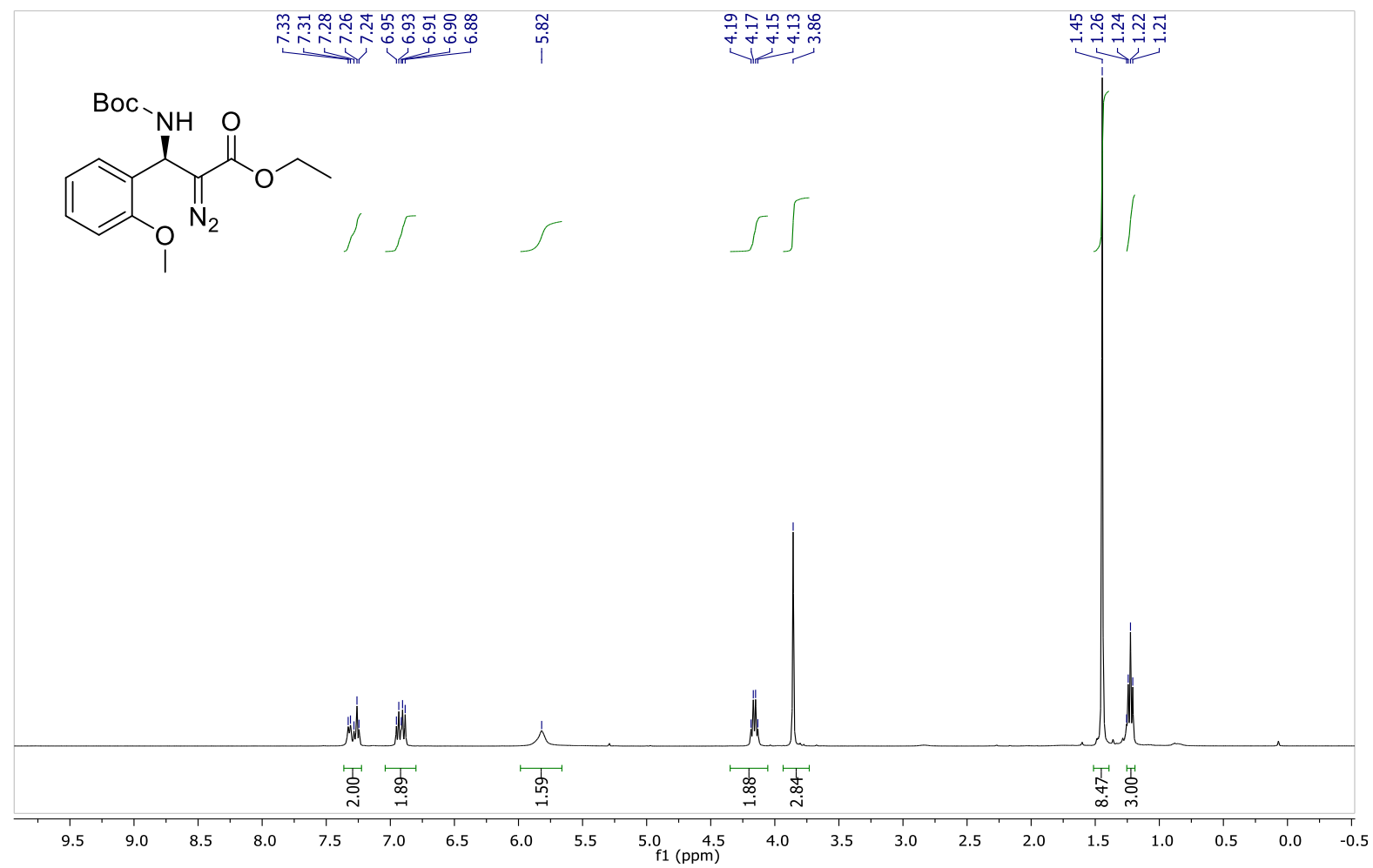

${ }^{13} \mathrm{C}\left\{{ }^{1} \mathrm{H}\right\}$ NMR spectrum of $\mathbf{4 d}(100 \mathrm{MHz})$

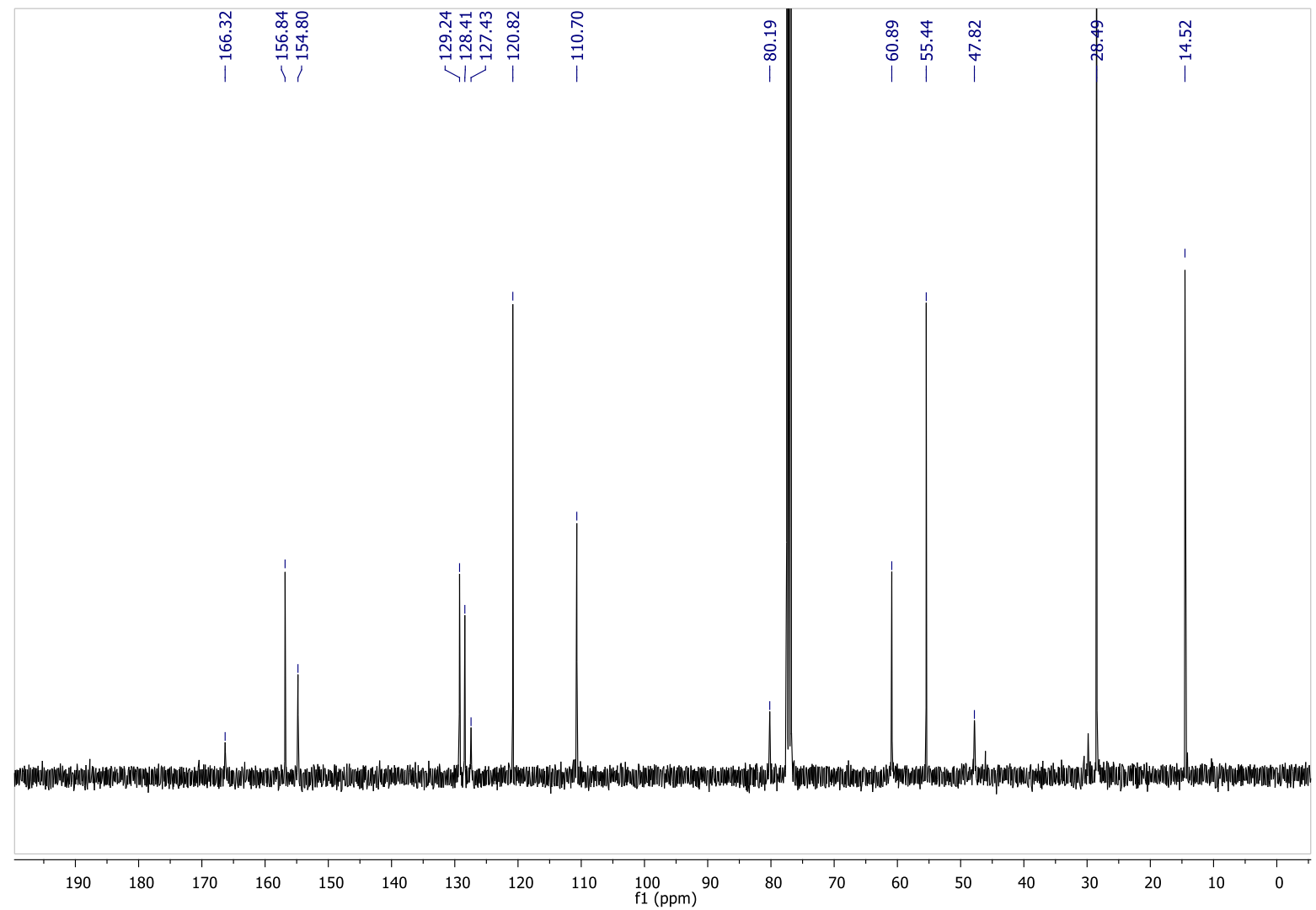


${ }^{1} \mathrm{H}-\mathrm{NMR}$ of $4 \mathbf{e}(400 \mathrm{MHz})$

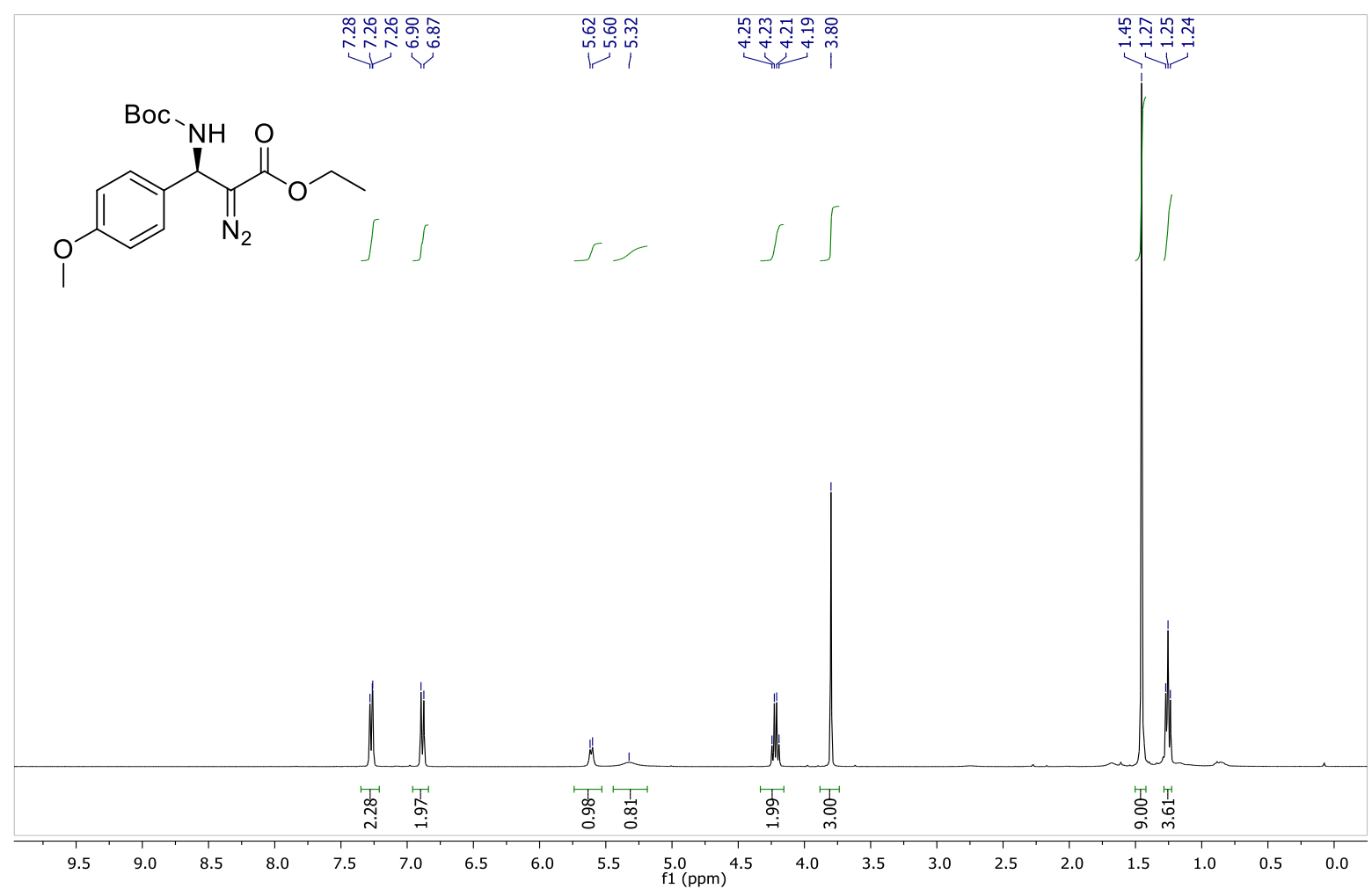

${ }^{13} \mathrm{C}\left\{{ }^{1} \mathrm{H}\right\}$ NMR of $4 \mathbf{e}(100 \mathrm{MHz})$

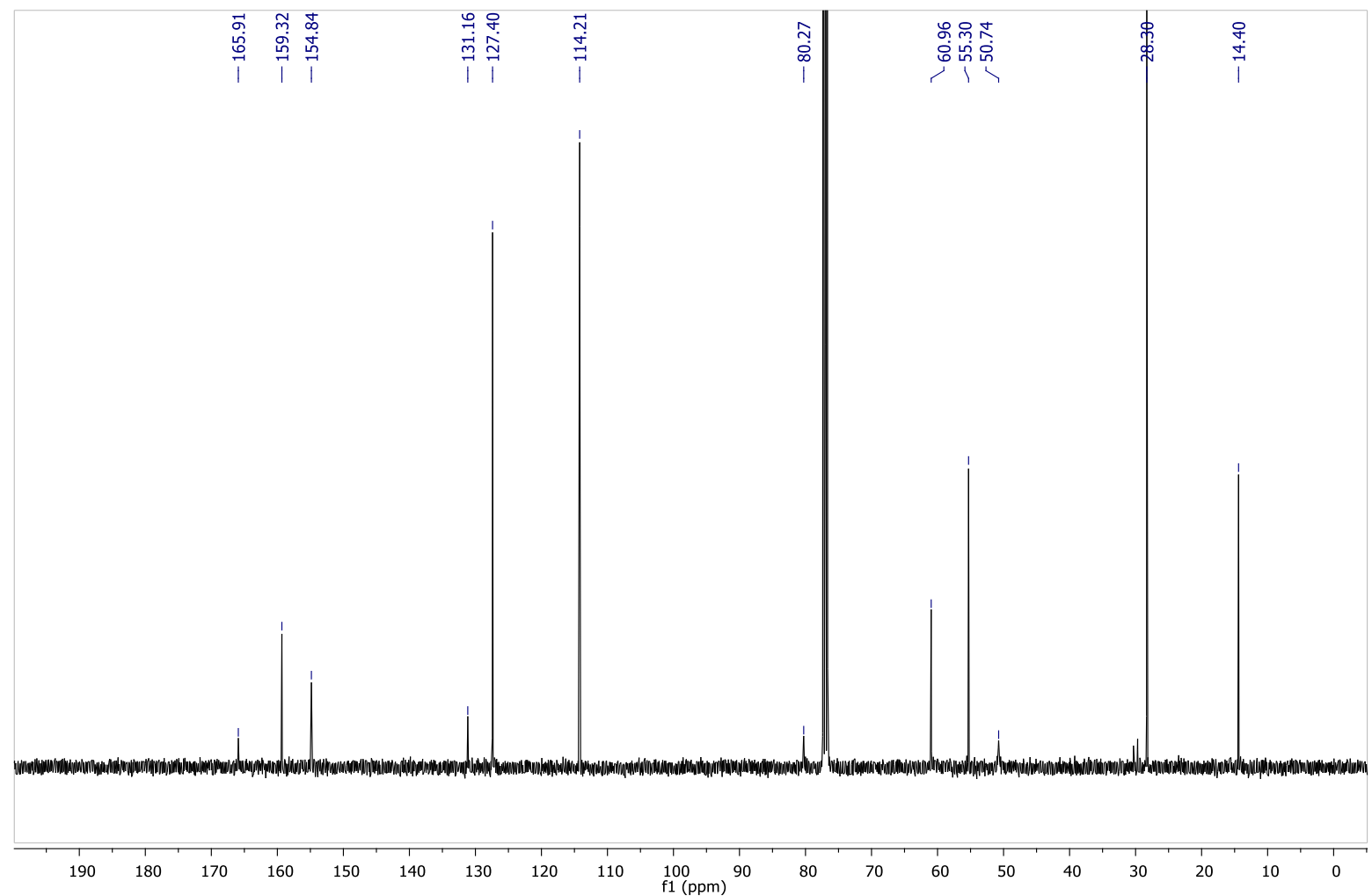


${ }^{1} \mathrm{H}-\mathrm{NMR}$ spectrum of $\mathbf{4 f}(400 \mathrm{MHz}$ )

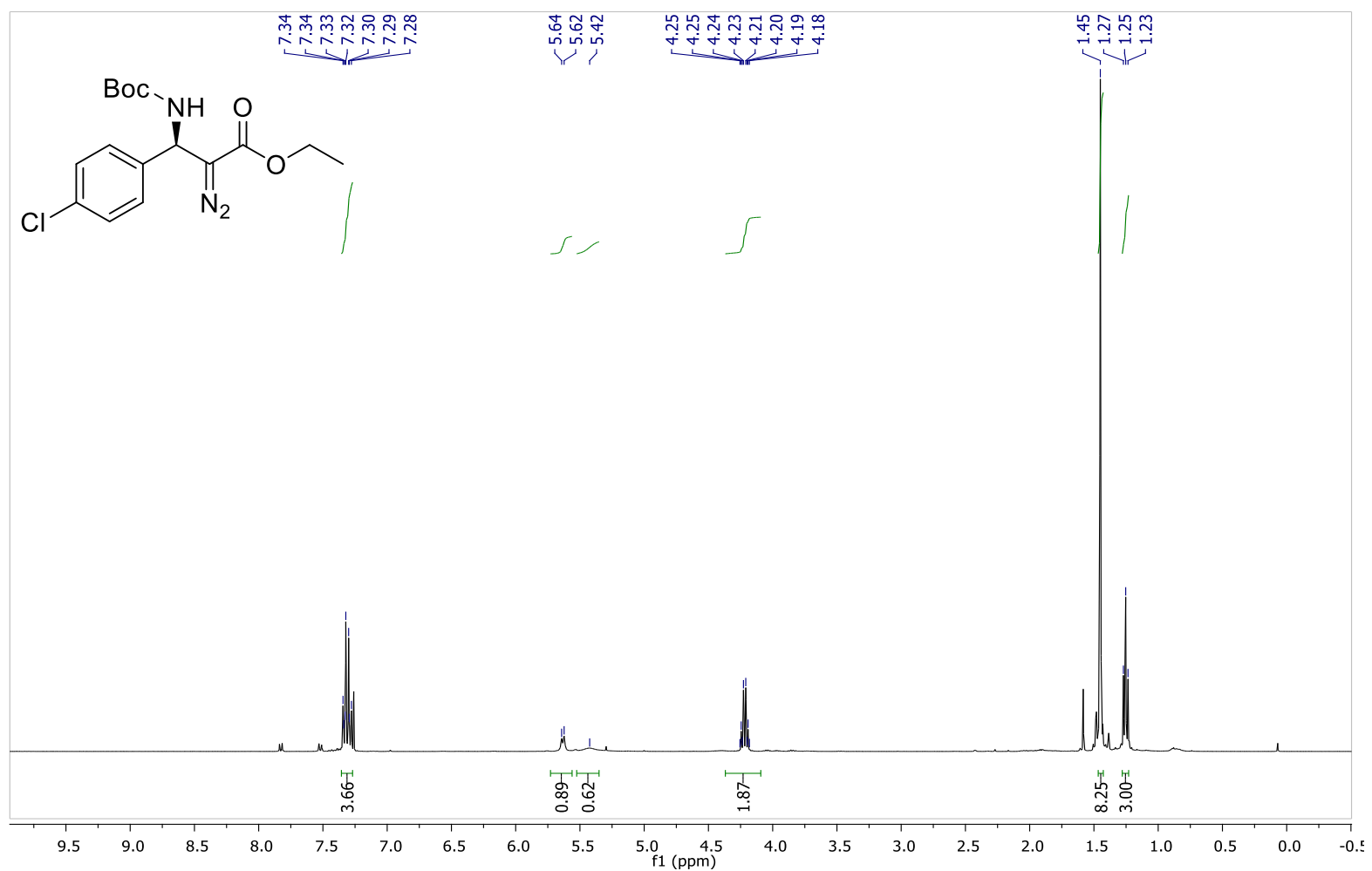

${ }^{13} \mathrm{C}\left\{{ }^{1} \mathrm{H}\right\}$ NMR spectrum of $\mathbf{4 f}(100 \mathrm{MHz})$

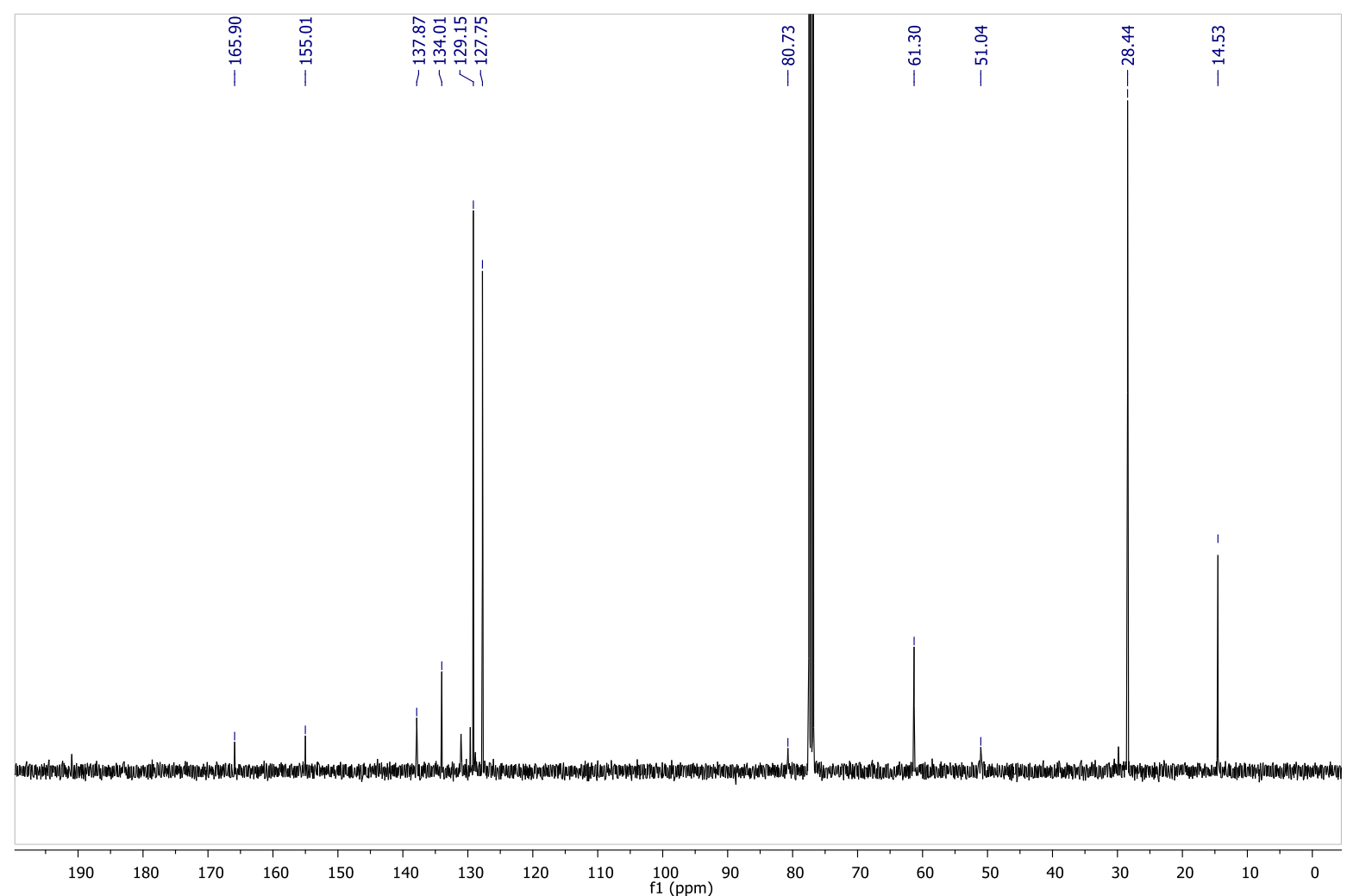


${ }^{1} \mathrm{H}-\mathrm{NMR}$ spectrum of $\mathbf{4 g}$ (400 MHz)

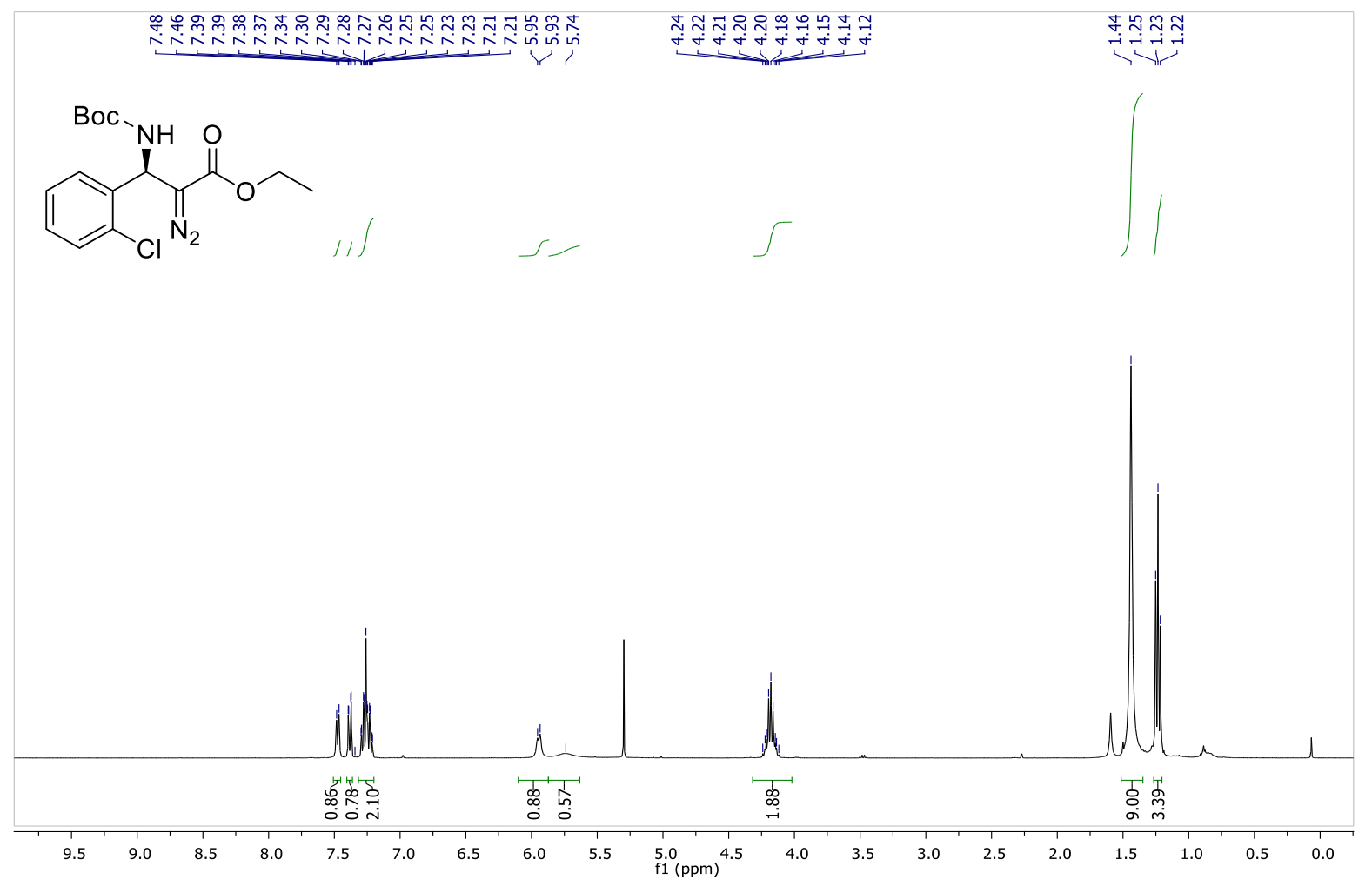

${ }^{13} \mathrm{C}\left\{{ }^{1} \mathrm{H}\right\}$ NMR spectrum of $\mathbf{4 g}(100 \mathrm{MHz})$

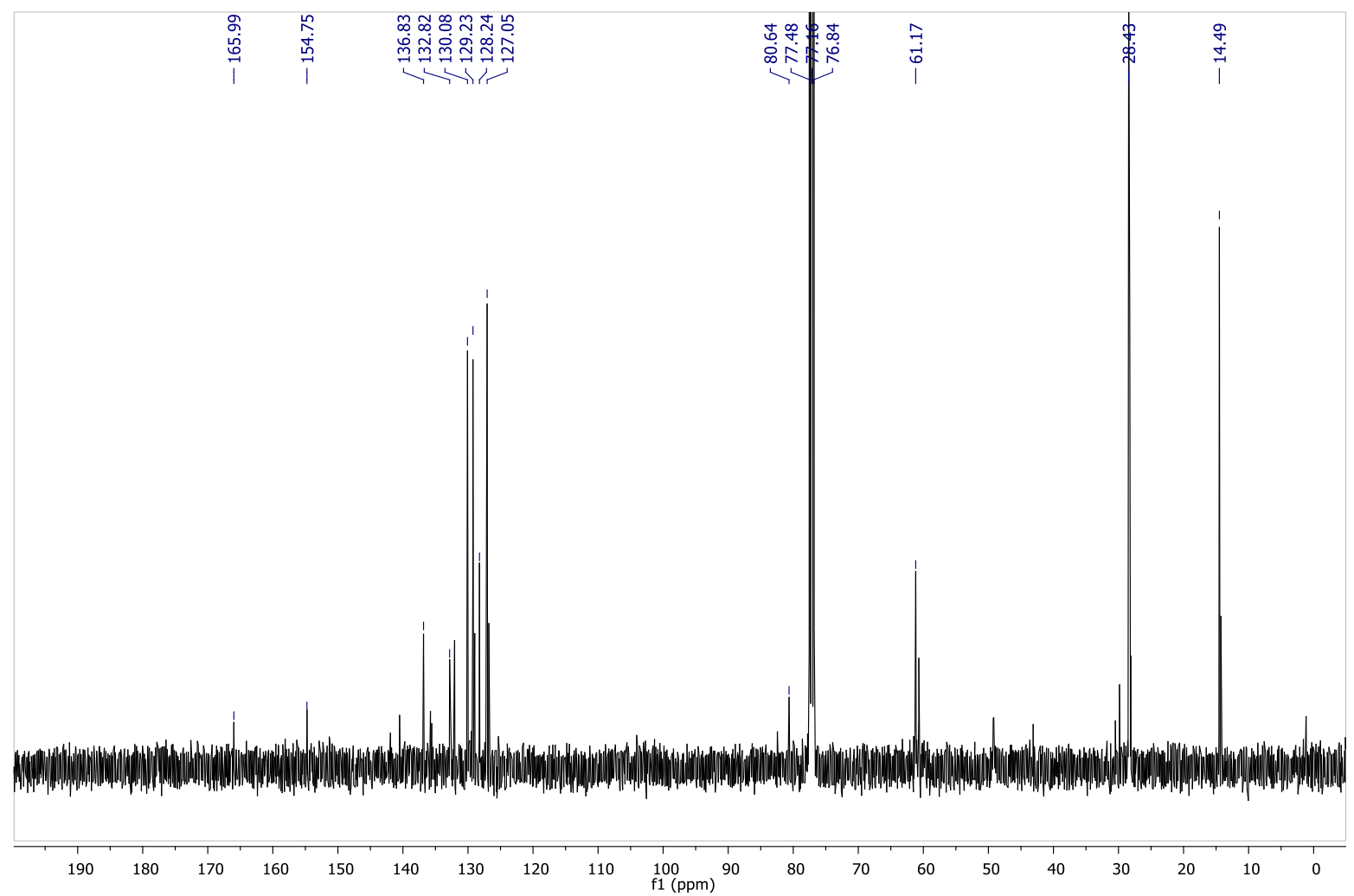


${ }^{1} \mathrm{H}-\mathrm{NMR}$ of $\mathbf{4 h}(400 \mathrm{MHz})$

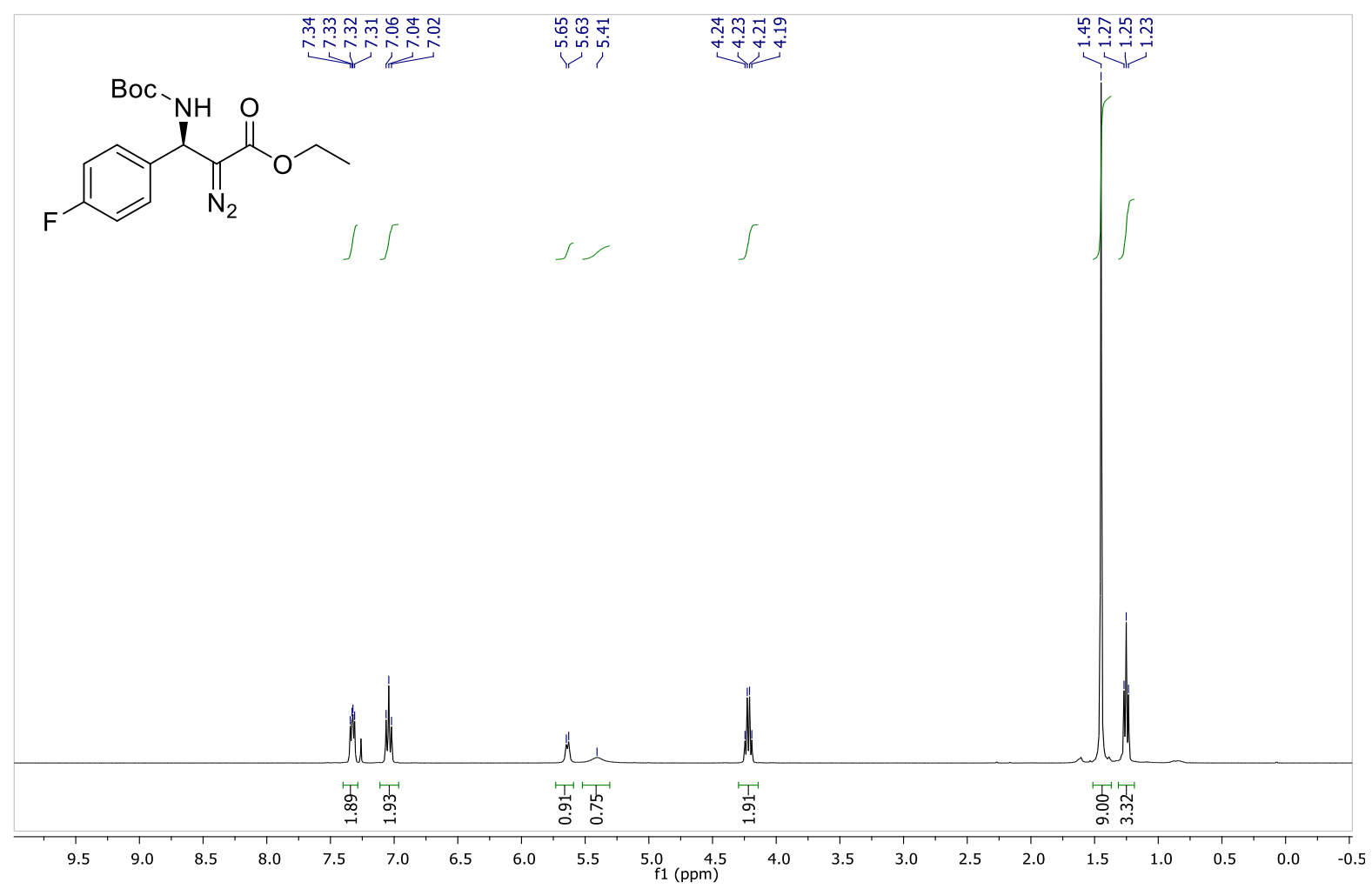

${ }^{13} \mathrm{C}\left\{{ }^{1} \mathrm{H}\right\}$ NMR of $\mathbf{4 h}(100 \mathrm{MHz})$

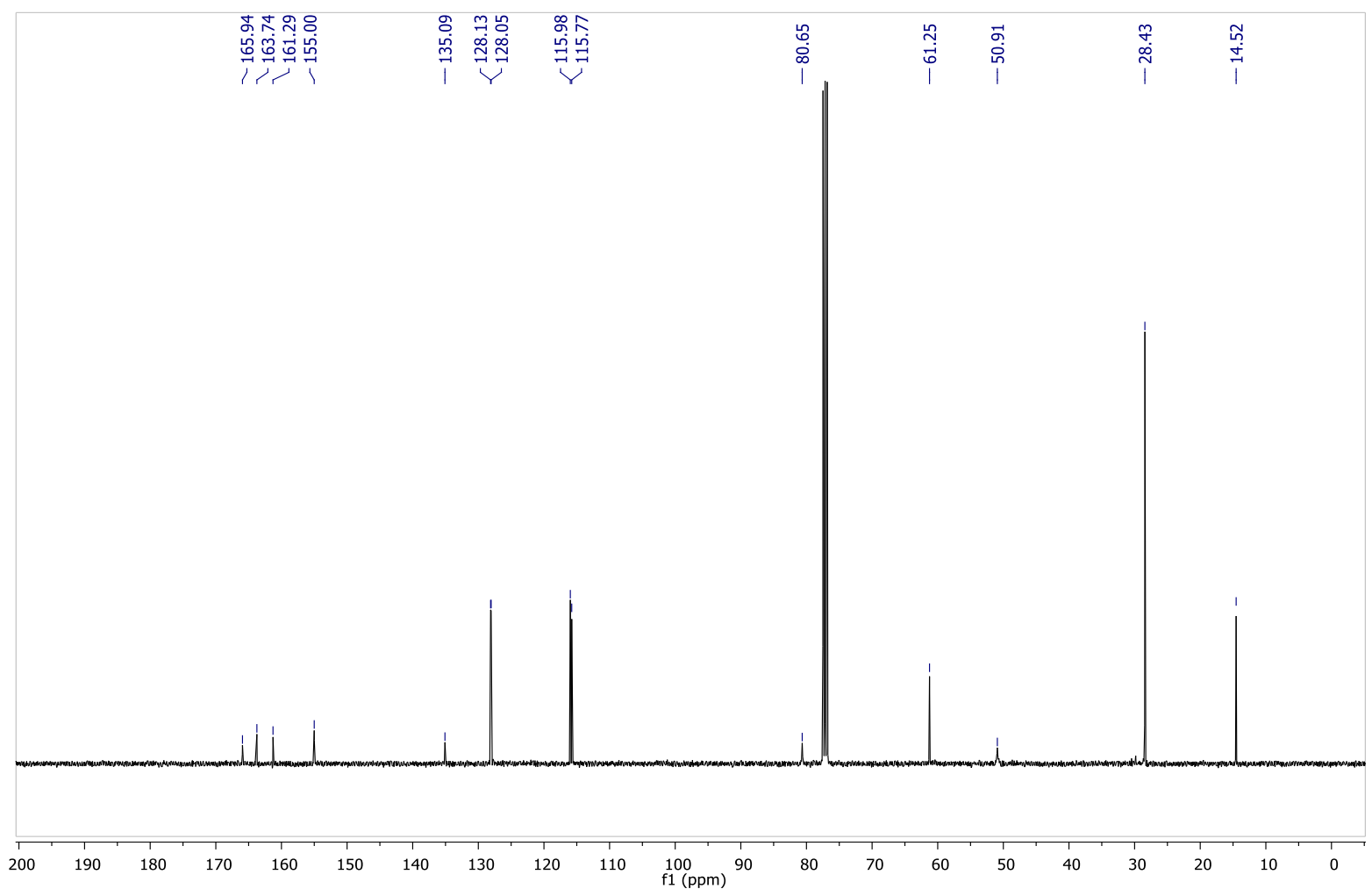


${ }^{1} \mathrm{H}-\mathrm{NMR}$ spectrum of $4 \mathbf{i}(400 \mathrm{MHz})$

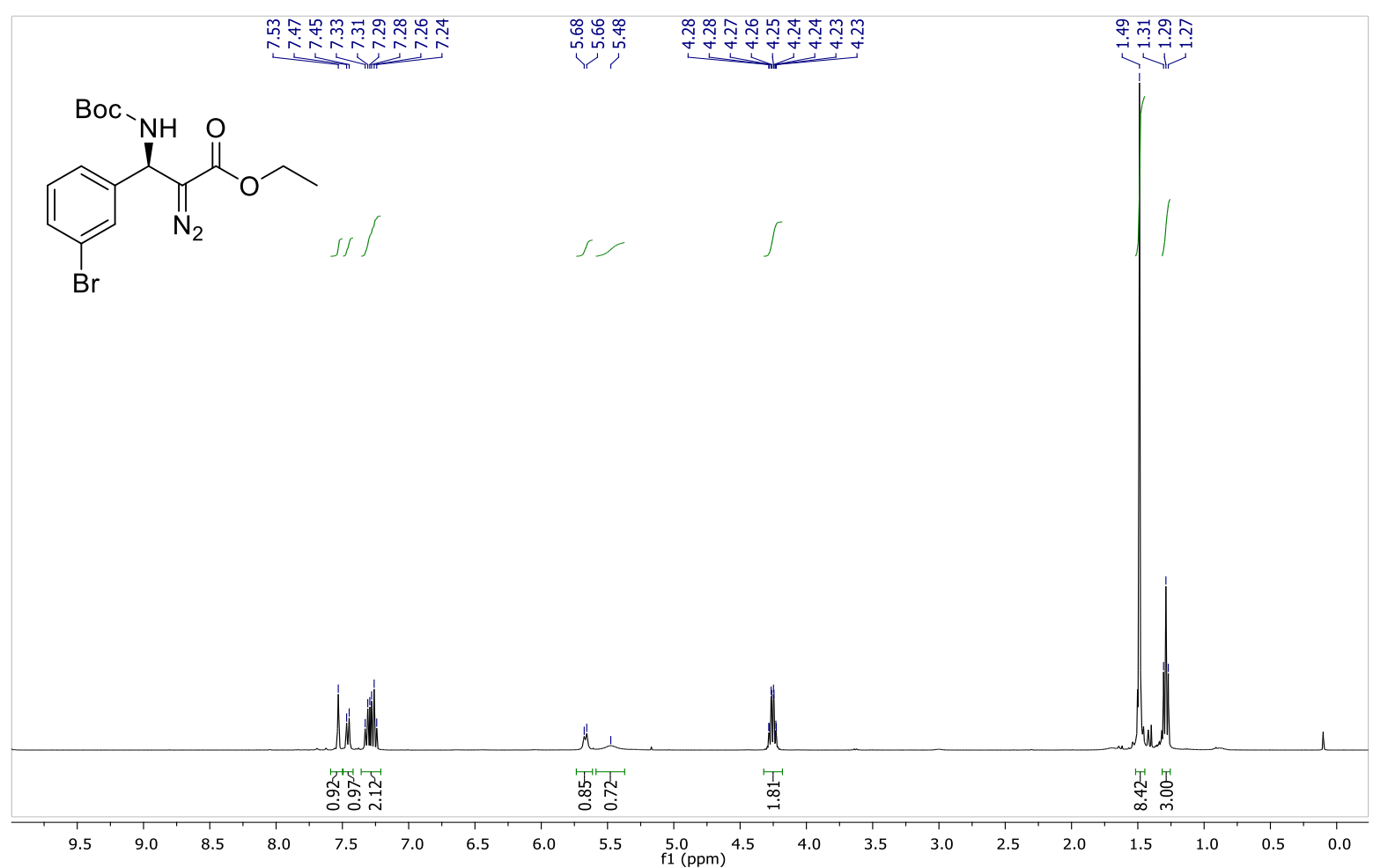

${ }^{13} \mathrm{C}\left\{{ }^{1} \mathrm{H}\right\}$ NMR spectrum of $4 \mathbf{i}(100 \mathrm{MHz})$

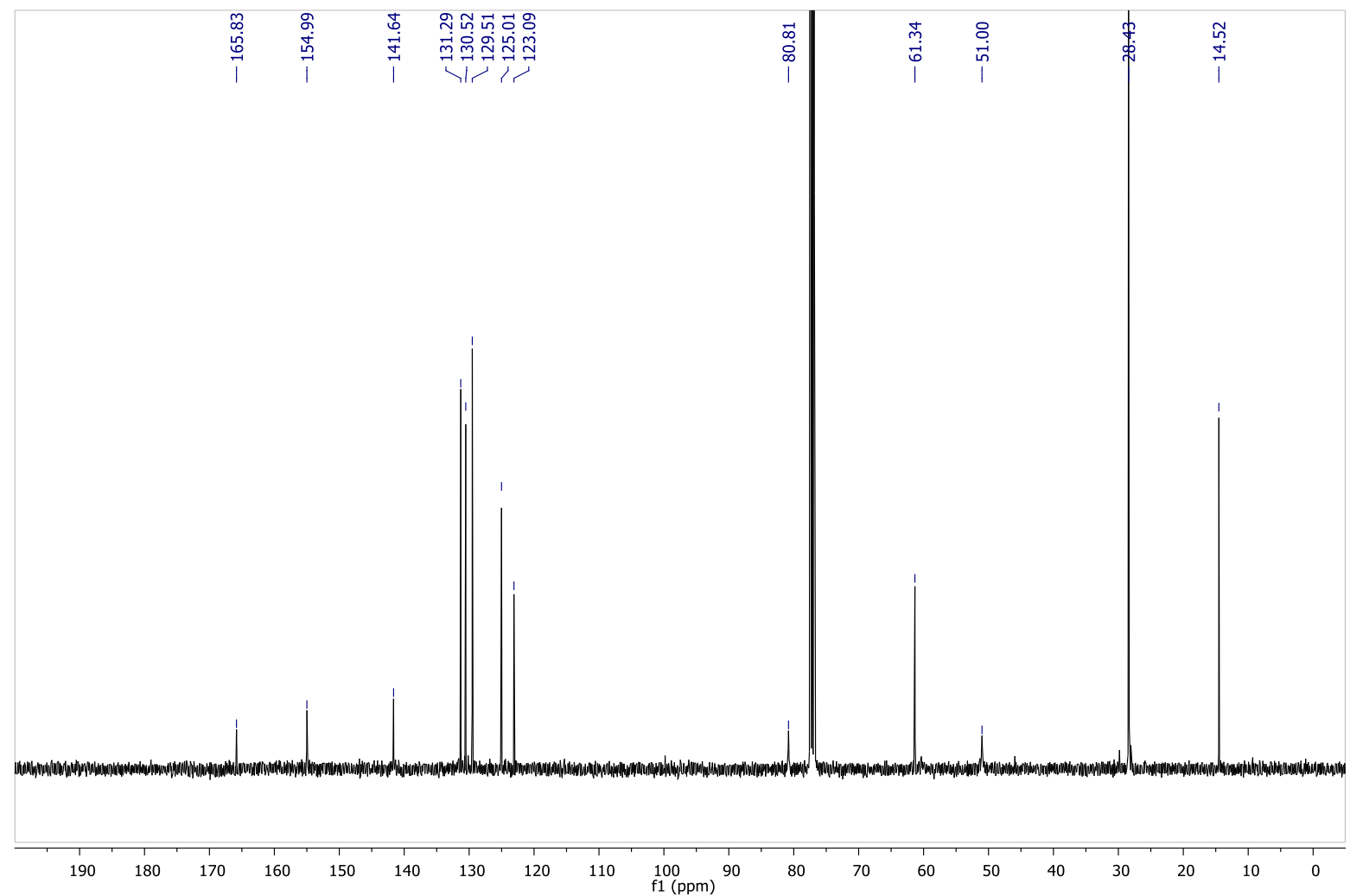


${ }^{1} \mathrm{H}-\mathrm{NMR}$ of $\mathbf{4 j}(400 \mathrm{MHz})$

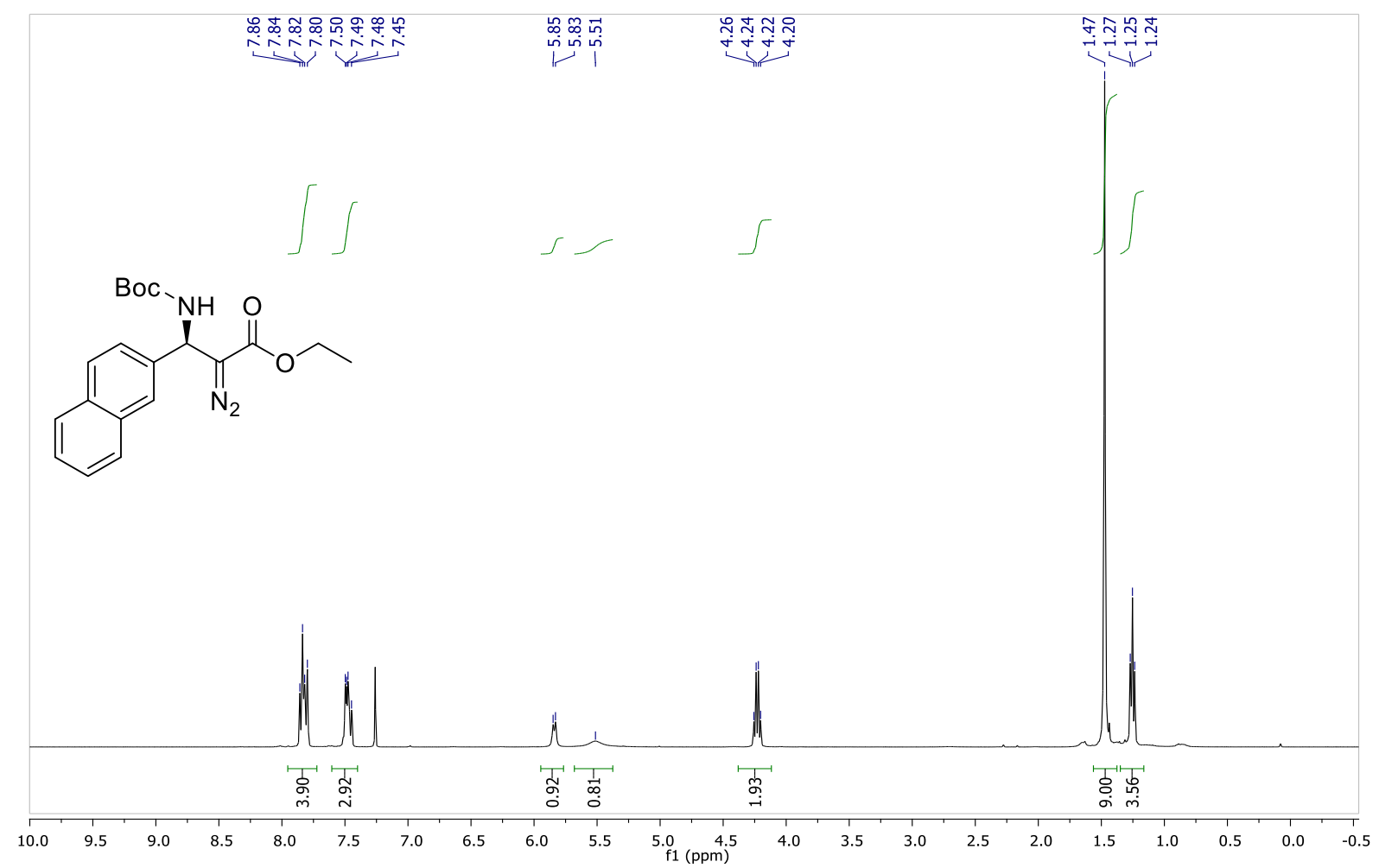

${ }^{13} \mathrm{C}\left\{{ }^{1} \mathrm{H}\right\}$ NMR of $4 \mathbf{j}(100 \mathrm{MHz})$

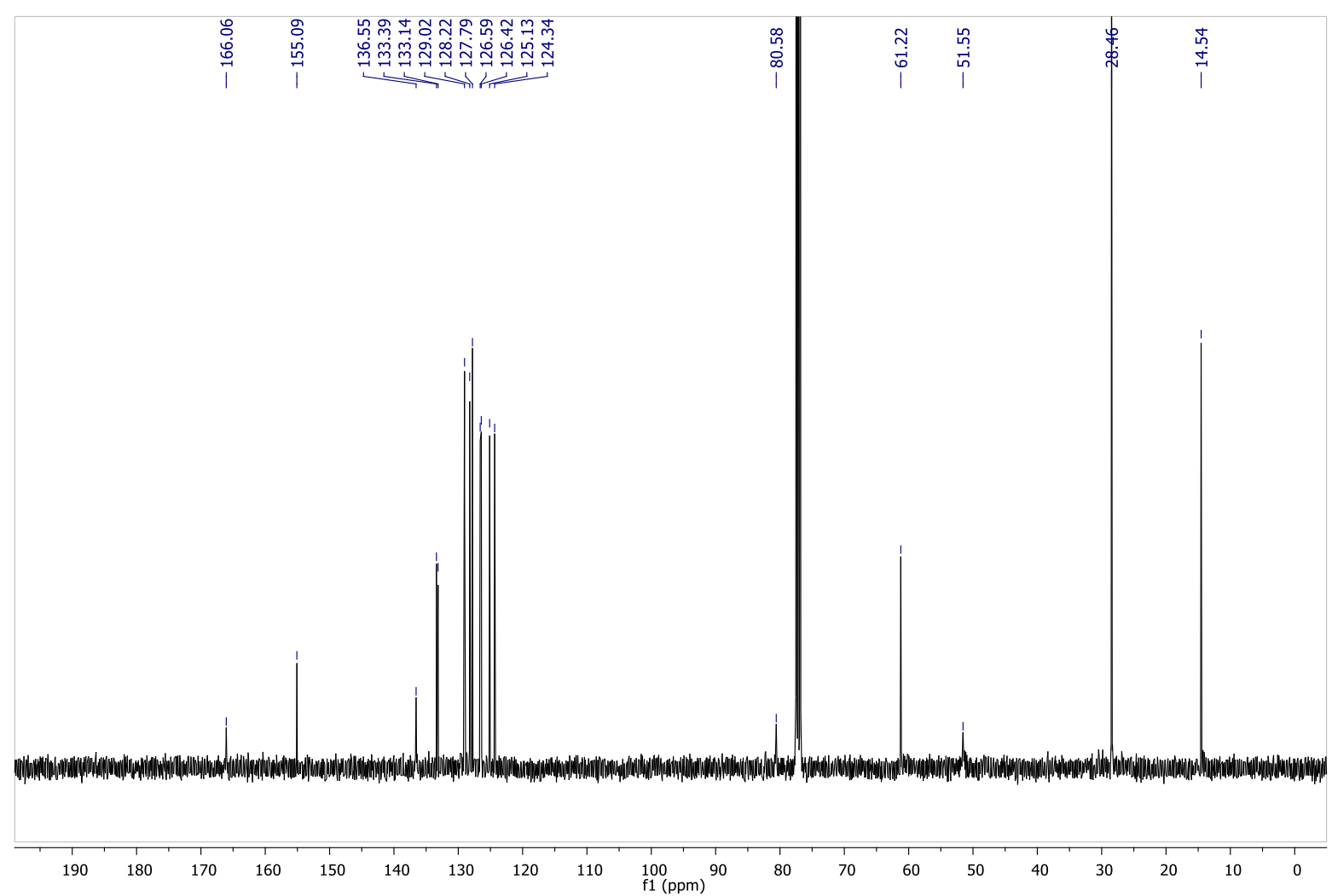


${ }^{1} \mathrm{H}-\mathrm{NMR}$ of $\mathbf{4 k}(400 \mathrm{MHz})$

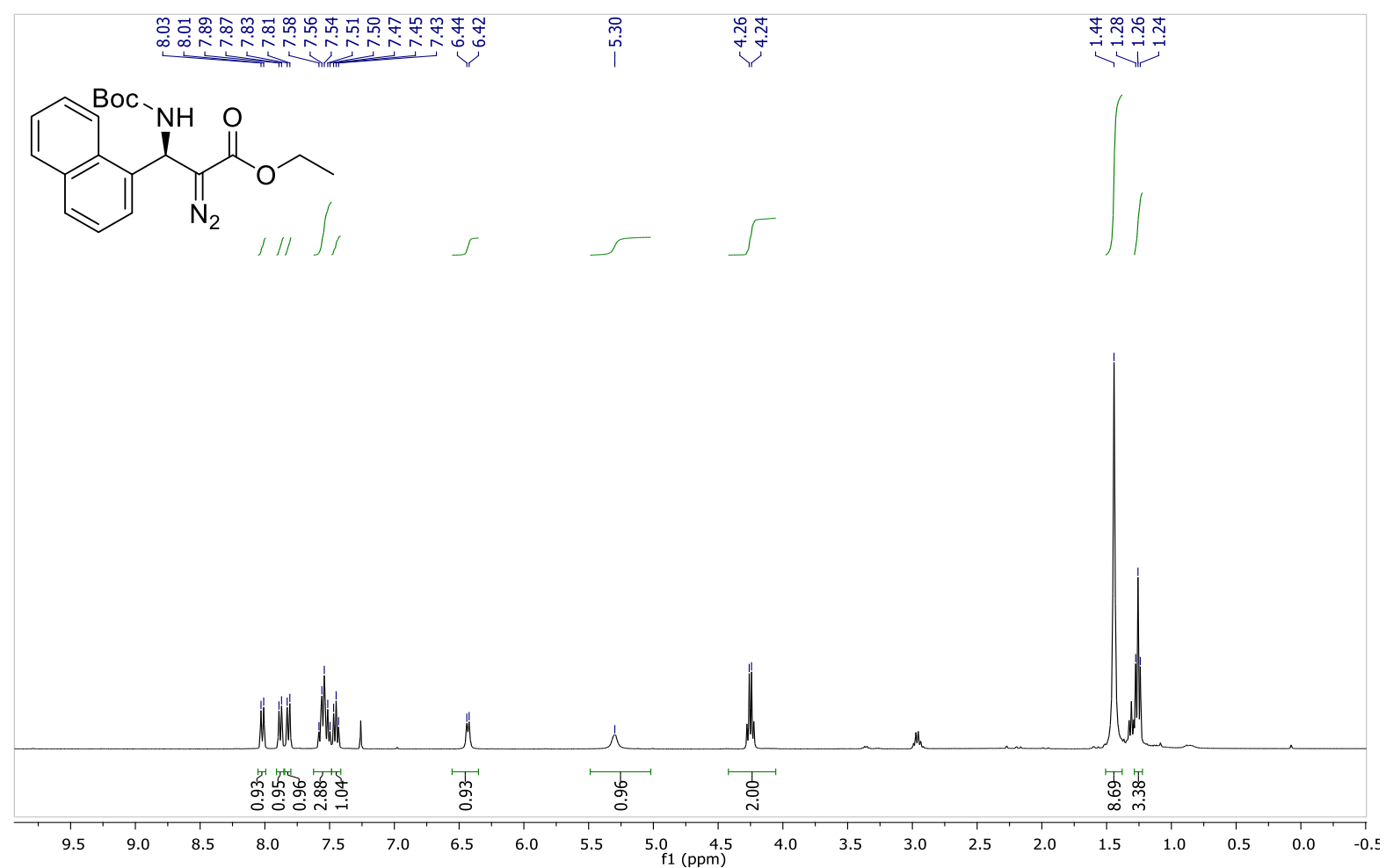

${ }^{13} \mathrm{C}\left\{{ }^{1} \mathrm{H}\right\}$ NMR spectrum of $\mathbf{4 k}(100 \mathrm{MHz})$

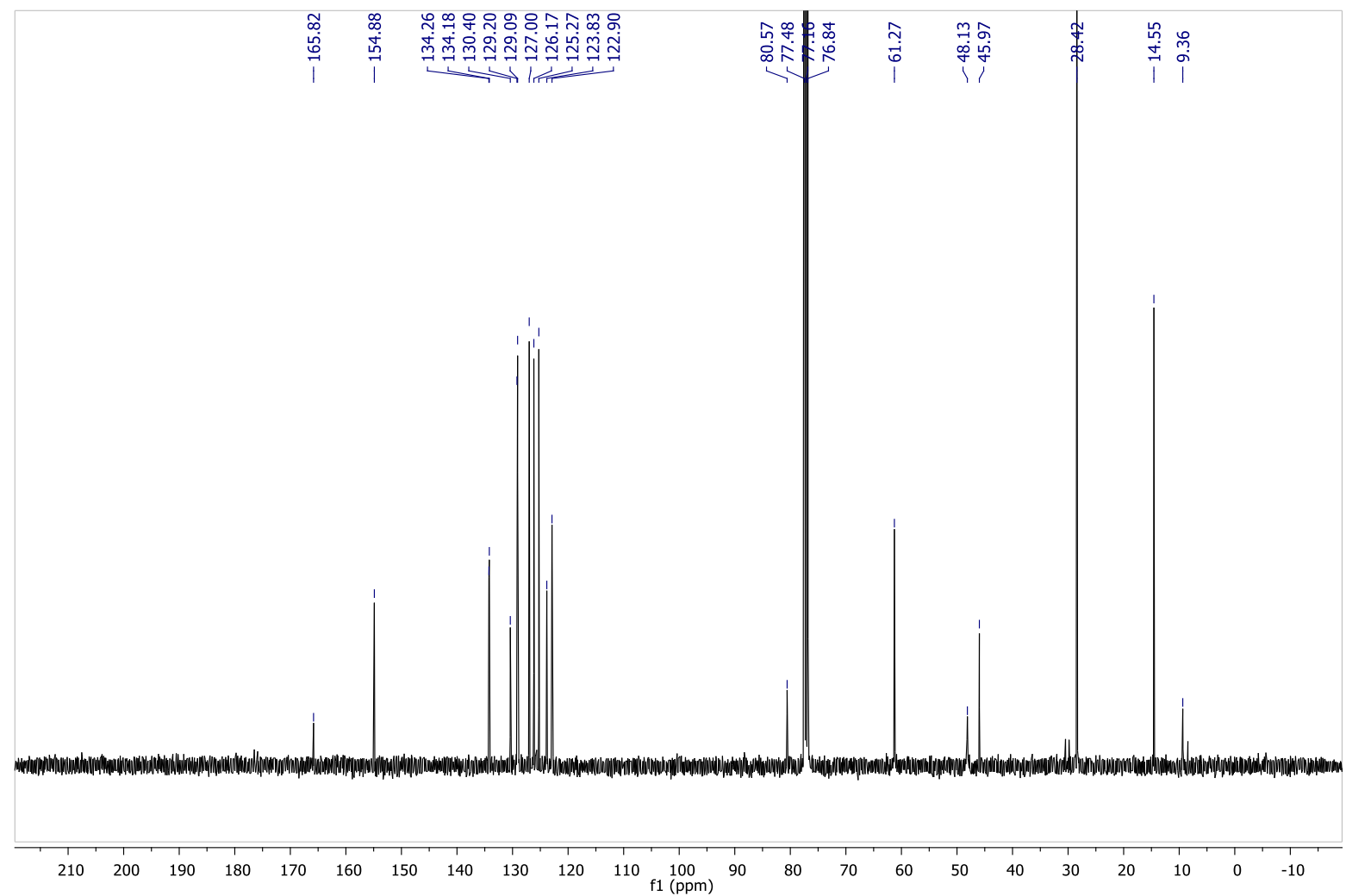


${ }^{1} \mathrm{H}-\mathrm{NMR}$ spectrum of 41 (400 MHz)

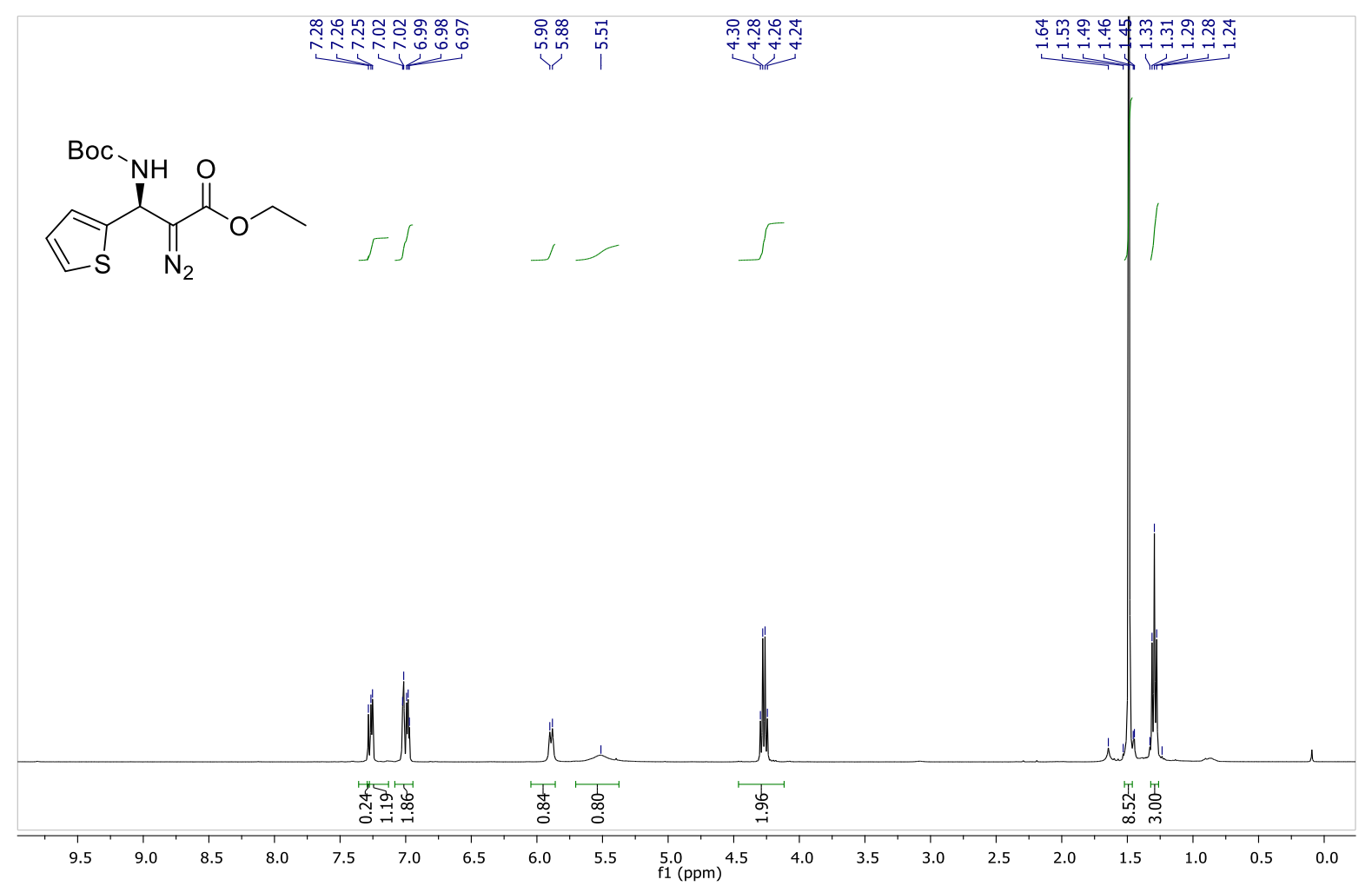

${ }^{13} \mathrm{C}\left\{{ }^{1} \mathrm{H}\right\}$ NMR spectrum of $4 \mathbf{l}(100 \mathrm{MHz})$

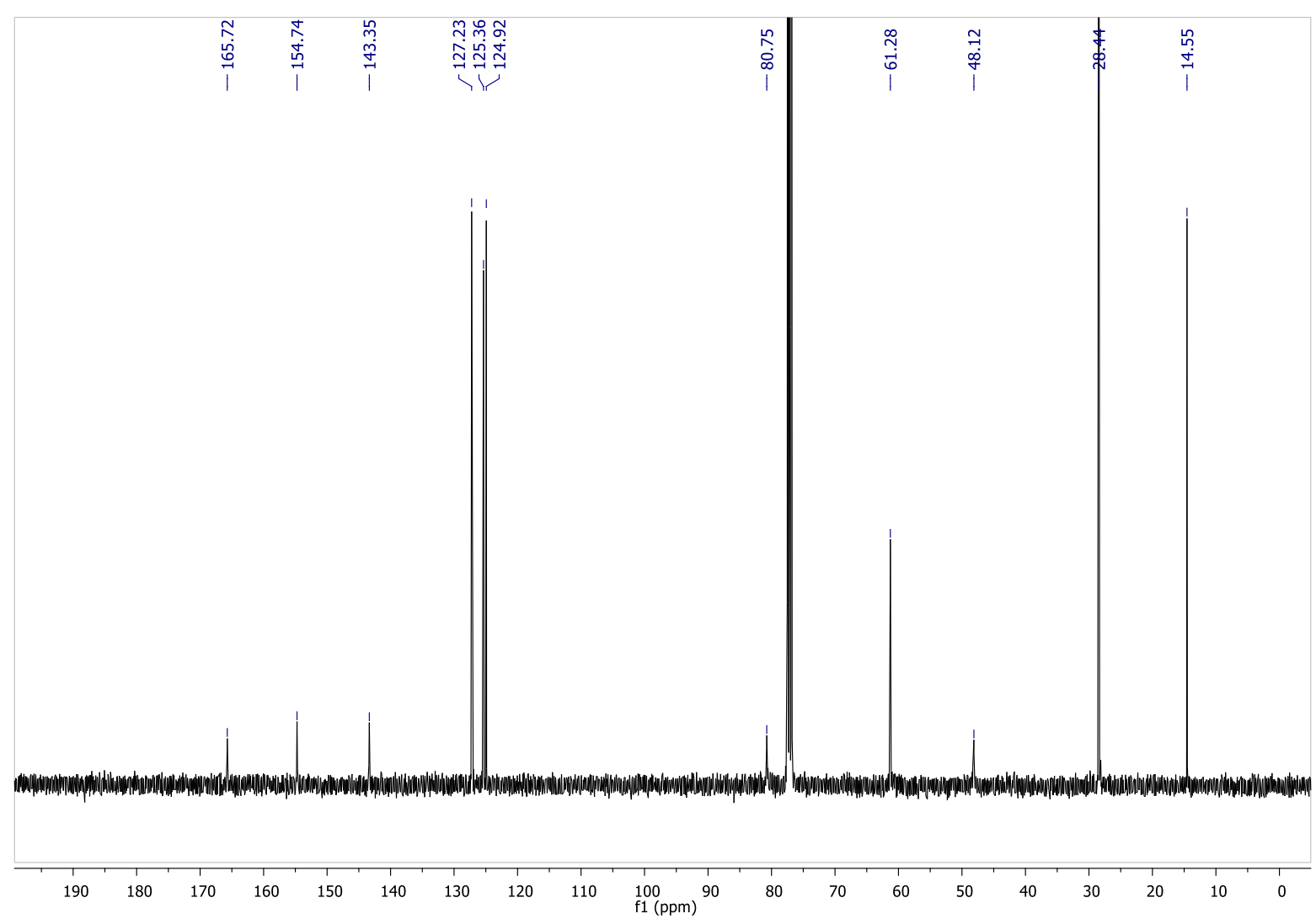


${ }^{1} \mathrm{H}-\mathrm{NMR}$ of $\mathbf{4 m}(400 \mathrm{MHz})$

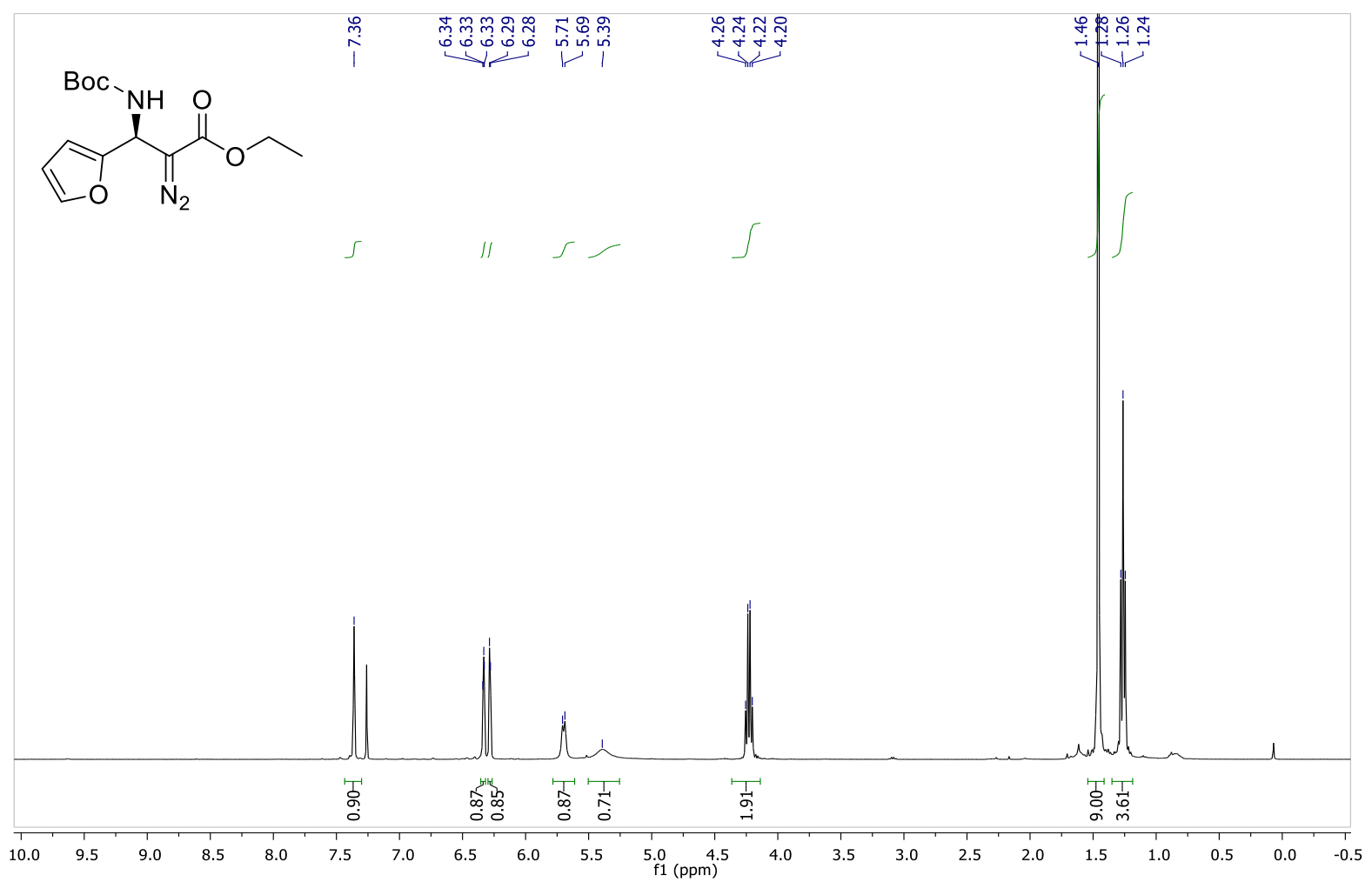

${ }^{13} \mathrm{C}\left\{{ }^{1} \mathrm{H}\right\}$ NMR of $\mathbf{4 m}(100 \mathrm{MHz})$

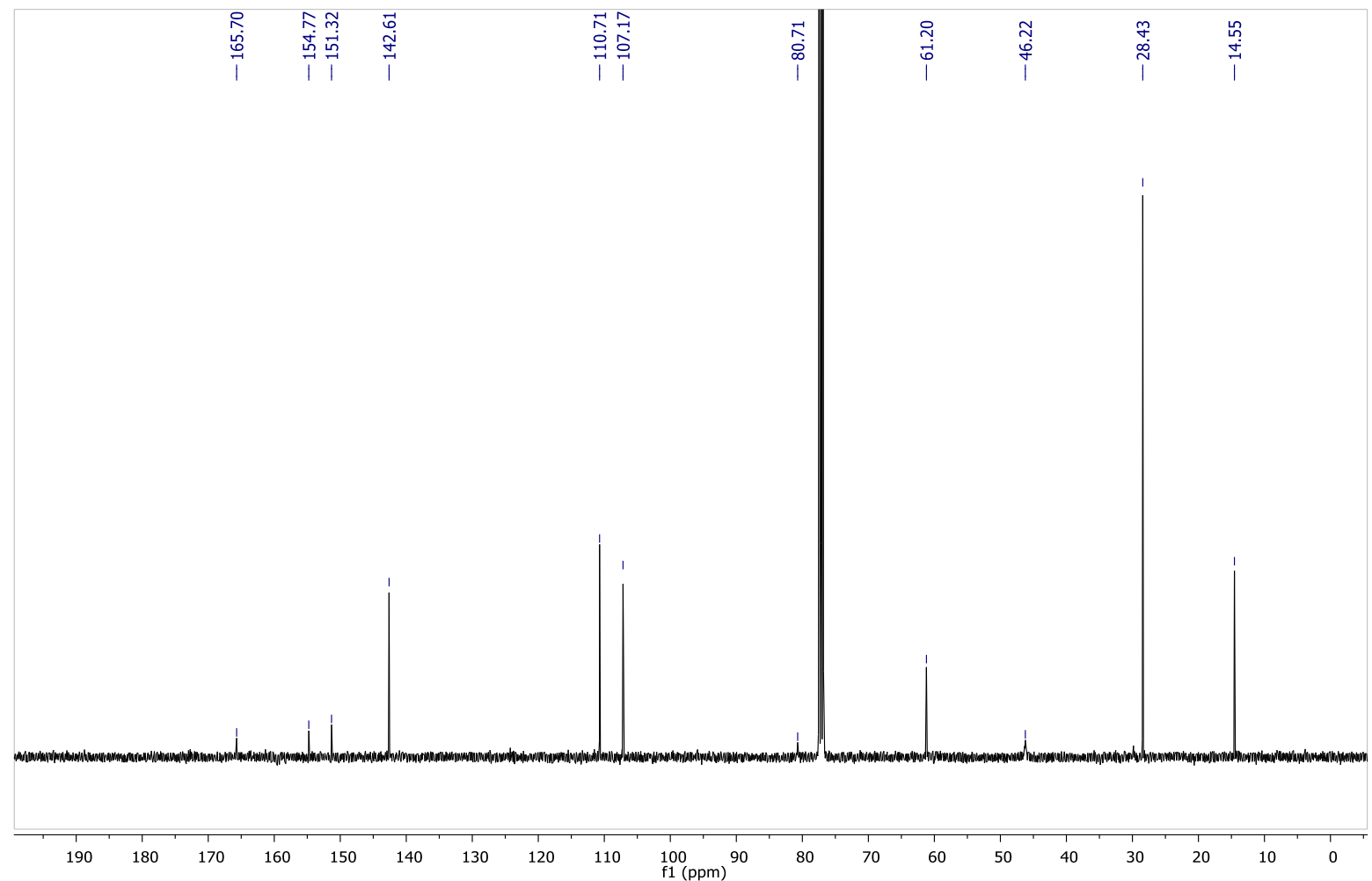


${ }^{1} \mathrm{H}-\mathrm{NMR}$ spectrum of $4 \mathbf{n}(500 \mathrm{MHz})$

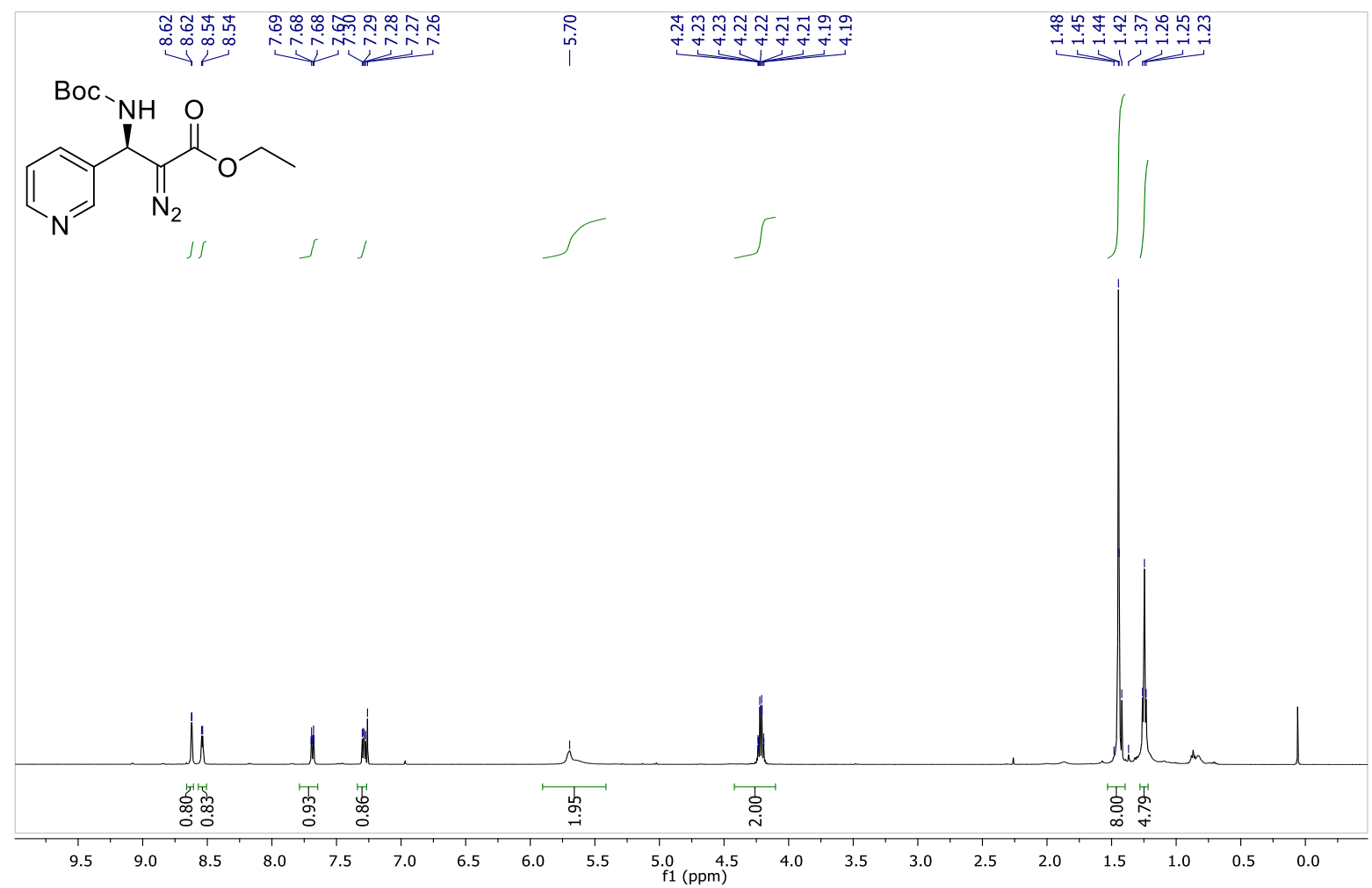

${ }^{13} \mathrm{C}\left\{{ }^{1} \mathrm{H}\right\}$ NMR spectrum of $4 \mathbf{n}(125 \mathrm{MHz})$

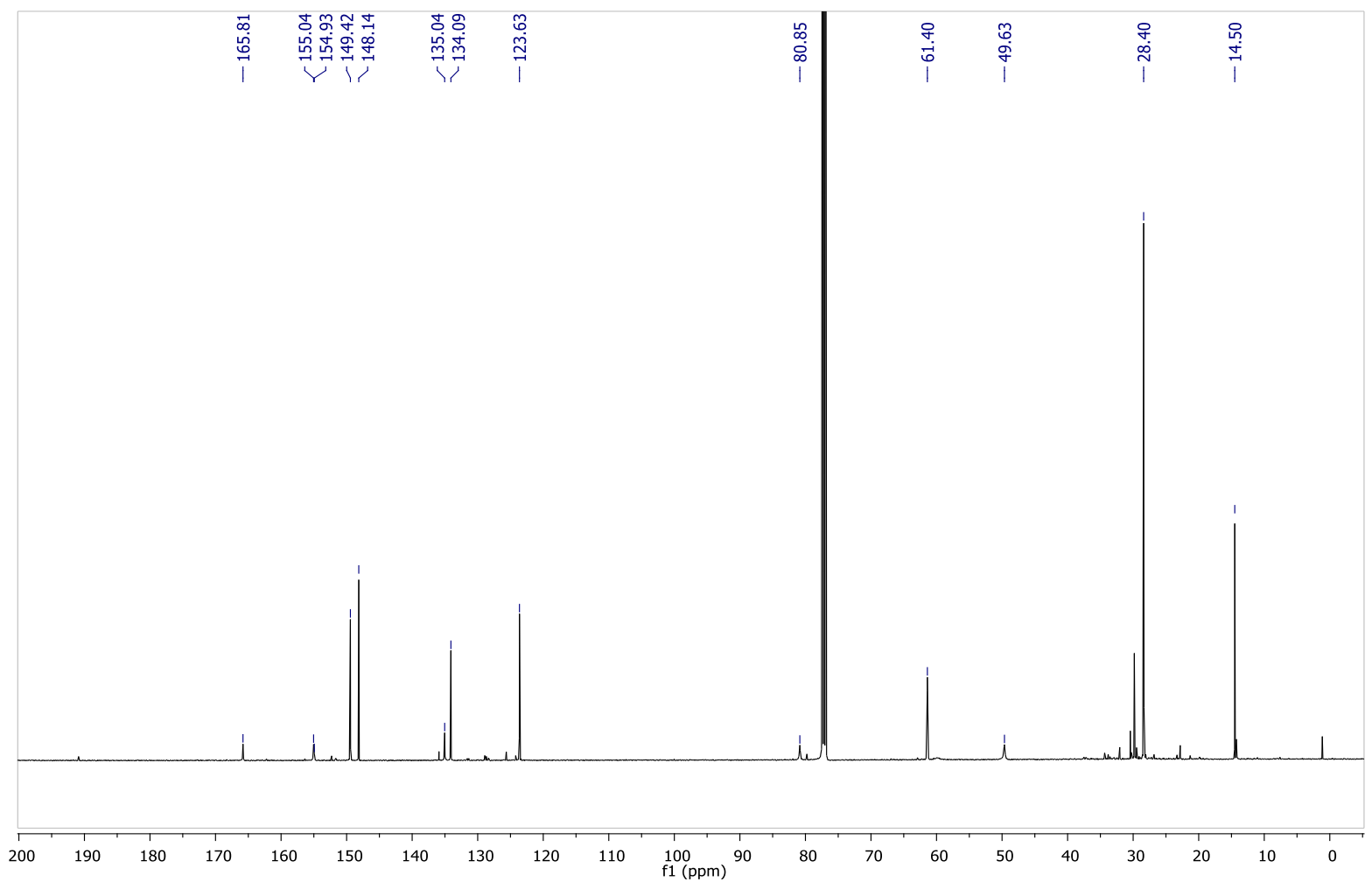


${ }^{1} \mathrm{H}-\mathrm{NMR}$ spectrum of $\mathbf{4 o}(500 \mathrm{MHz})$

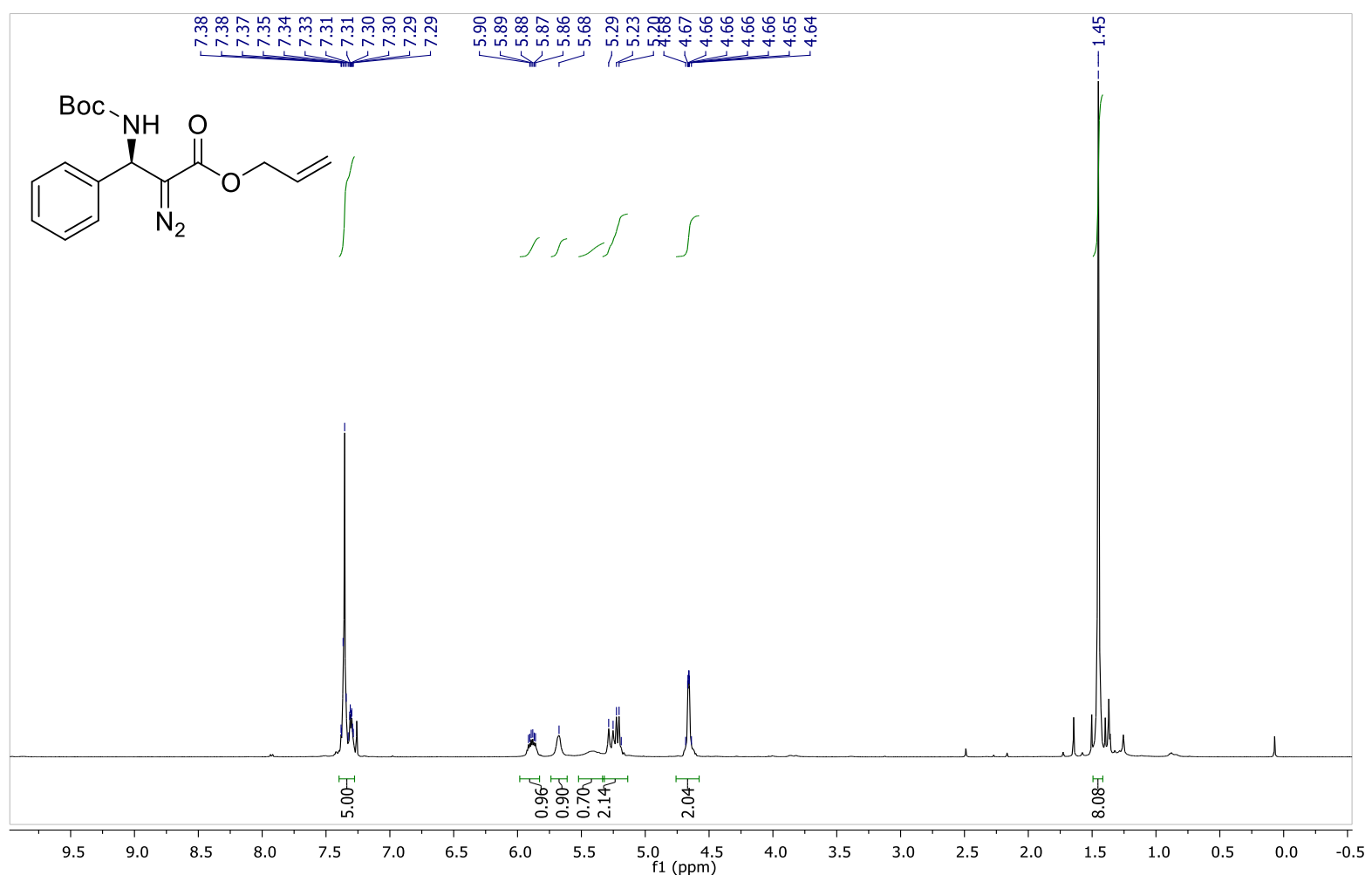

${ }^{13} \mathrm{C}\left\{{ }^{1} \mathrm{H}\right\}$ NMR spectrum of $40(125 \mathrm{MHz})$

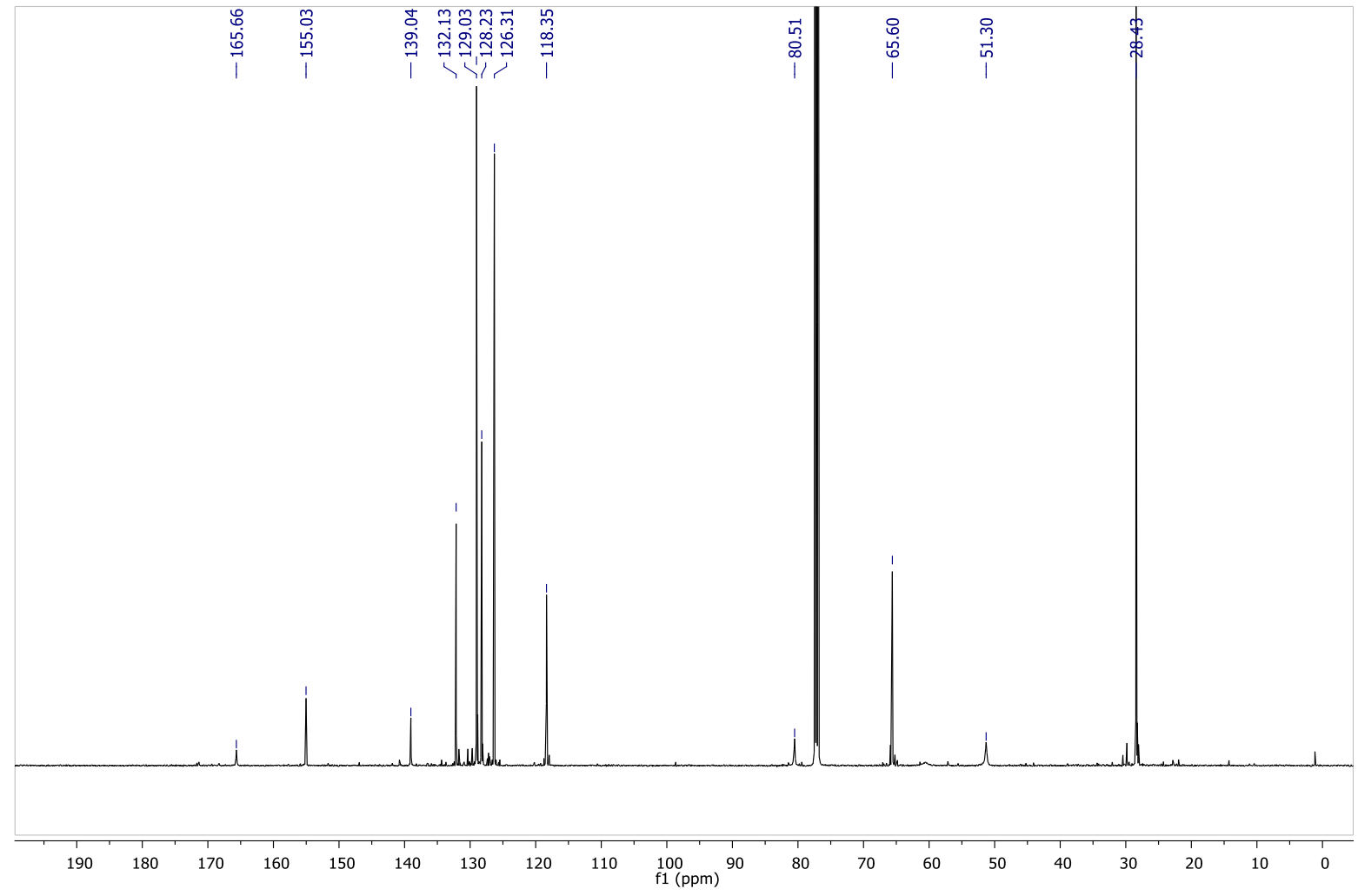


${ }^{1} \mathrm{H}-\mathrm{NMR}$ spectrum of $\mathbf{4 p}$ (400 MHz)

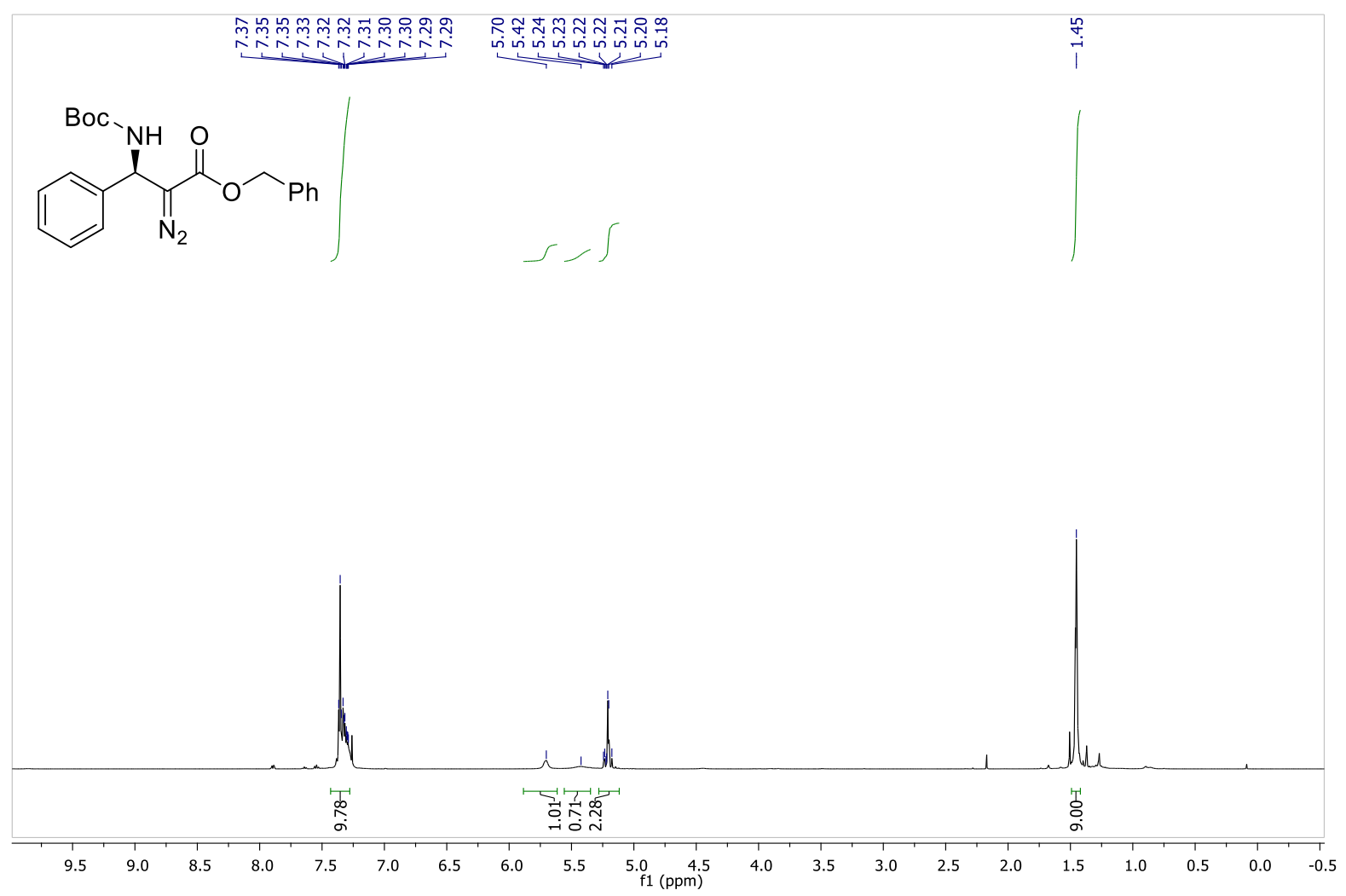

${ }^{13} \mathrm{C}\left\{{ }^{1} \mathrm{H}\right\}$ NMR spectrum of $\mathbf{4 p}(125 \mathrm{MHz})$

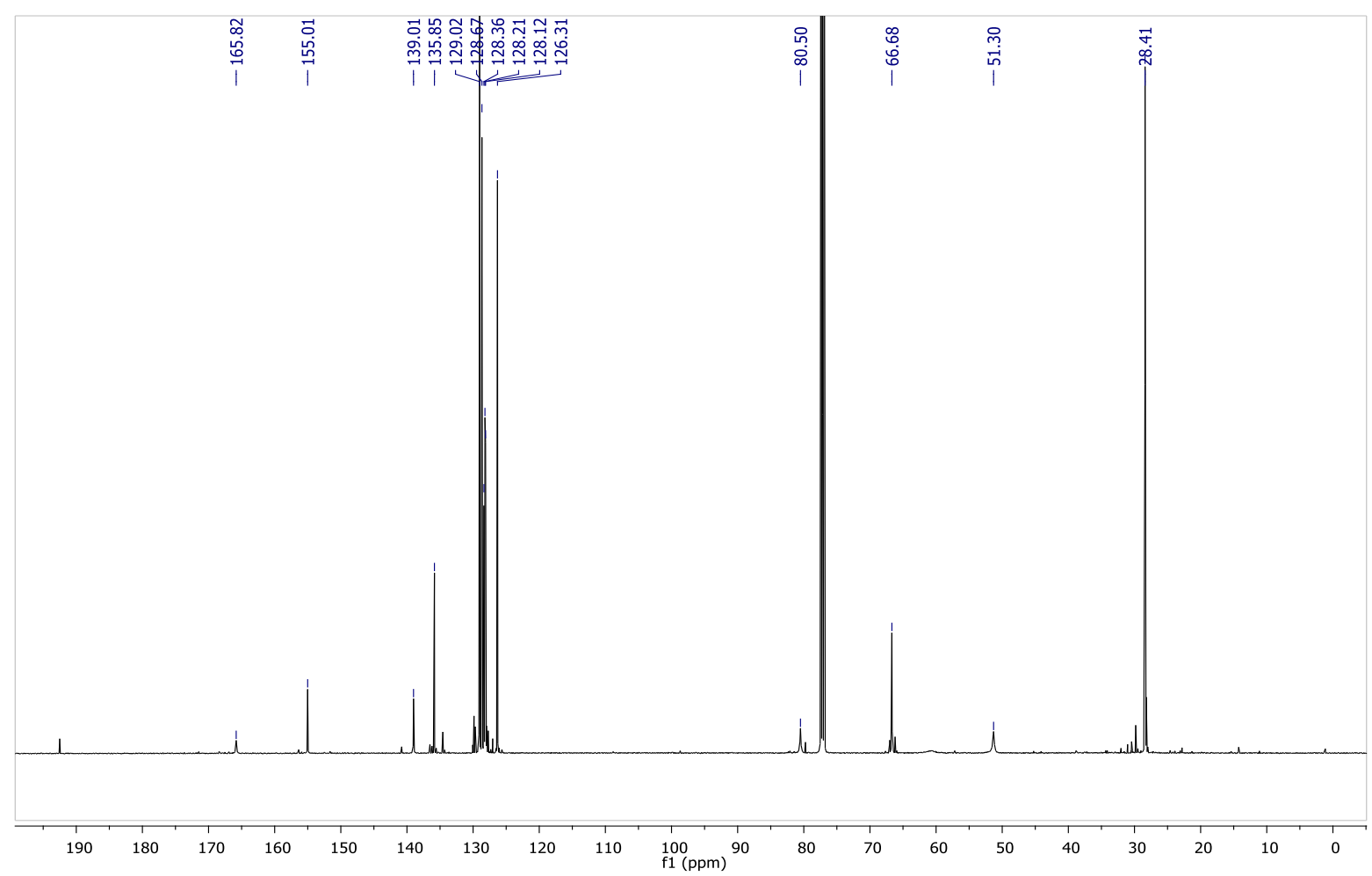


${ }^{1} \mathrm{H}-\mathrm{NMR}$ spectrum of $\mathbf{4 q}$ (400 MHz)

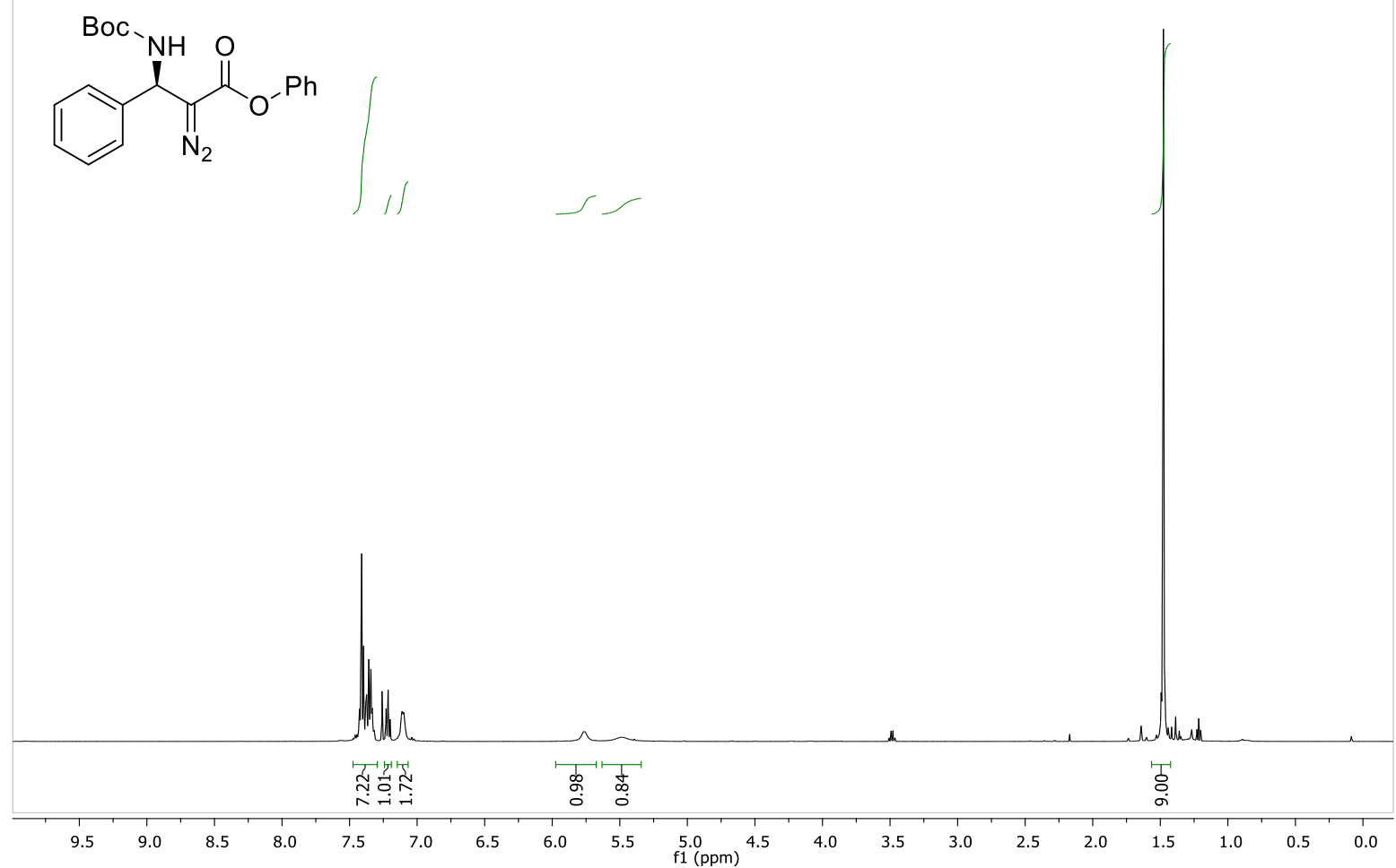

${ }^{13} \mathrm{C}\left\{{ }^{1} \mathrm{H}\right\}$ NMR spectrum of $\mathbf{4 q}(125 \mathrm{MHz})$

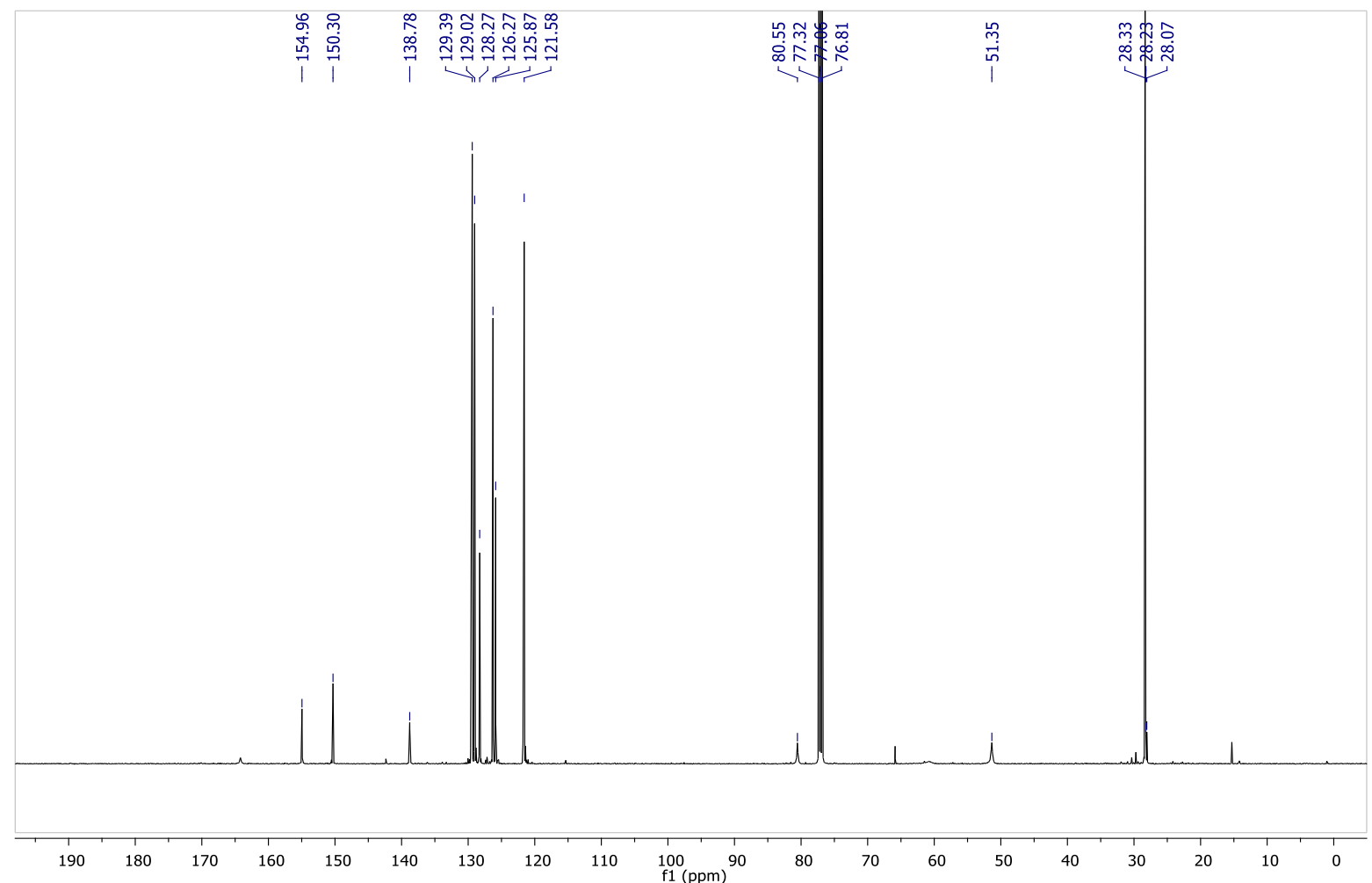


${ }^{1} \mathrm{H}-\mathrm{NMR}$ spectrum of $\mathbf{5 a}(400 \mathrm{MHz})$

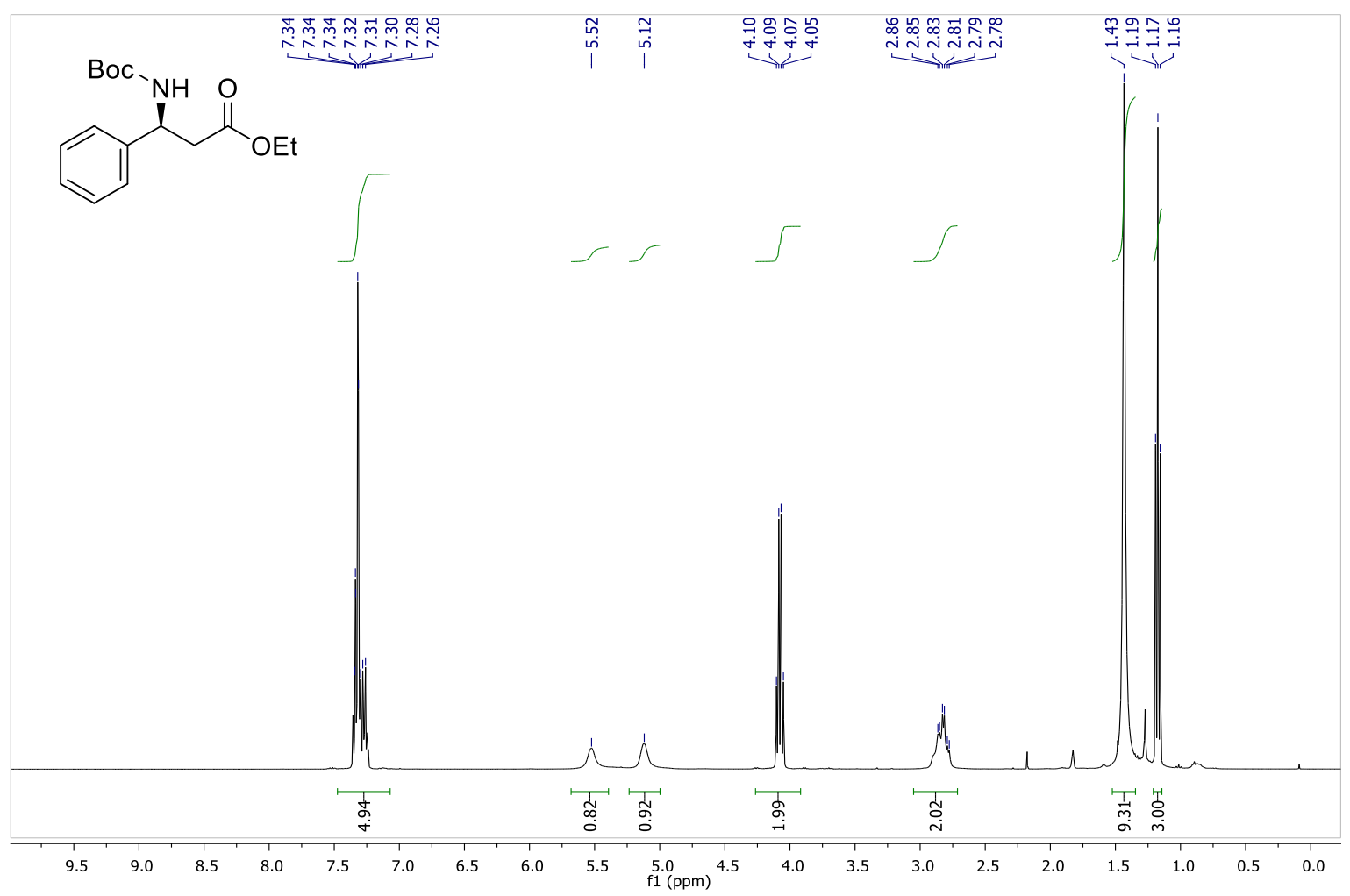

${ }^{13} \mathrm{C}\left\{{ }^{1} \mathrm{H}\right\}$ NMR spectrum of $\mathbf{5 a}(100 \mathrm{MHz})$

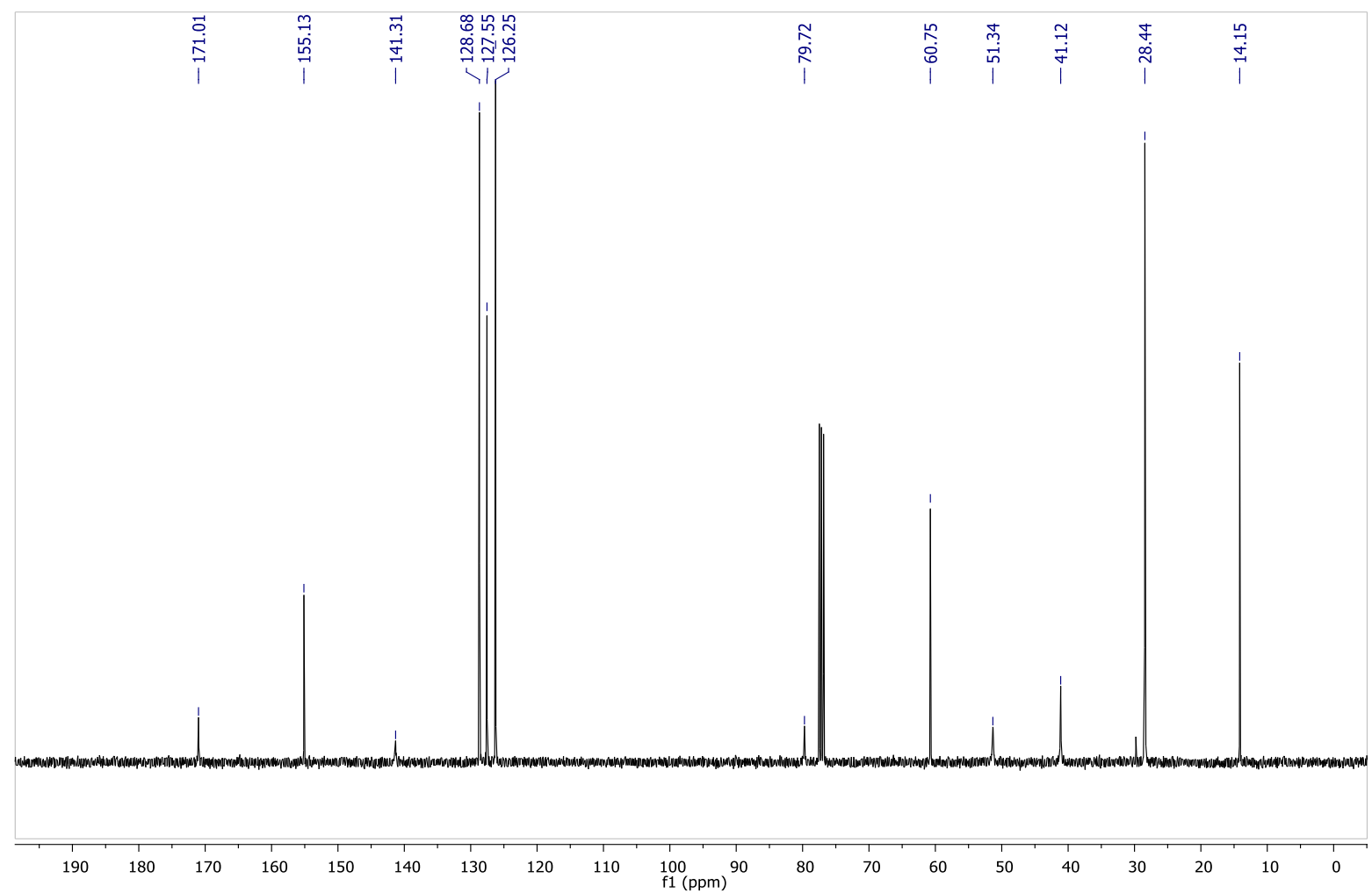


${ }^{1} \mathrm{H}-\mathrm{NMR}$ spectrum of $\mathbf{5 b}(400 \mathrm{MHz})$

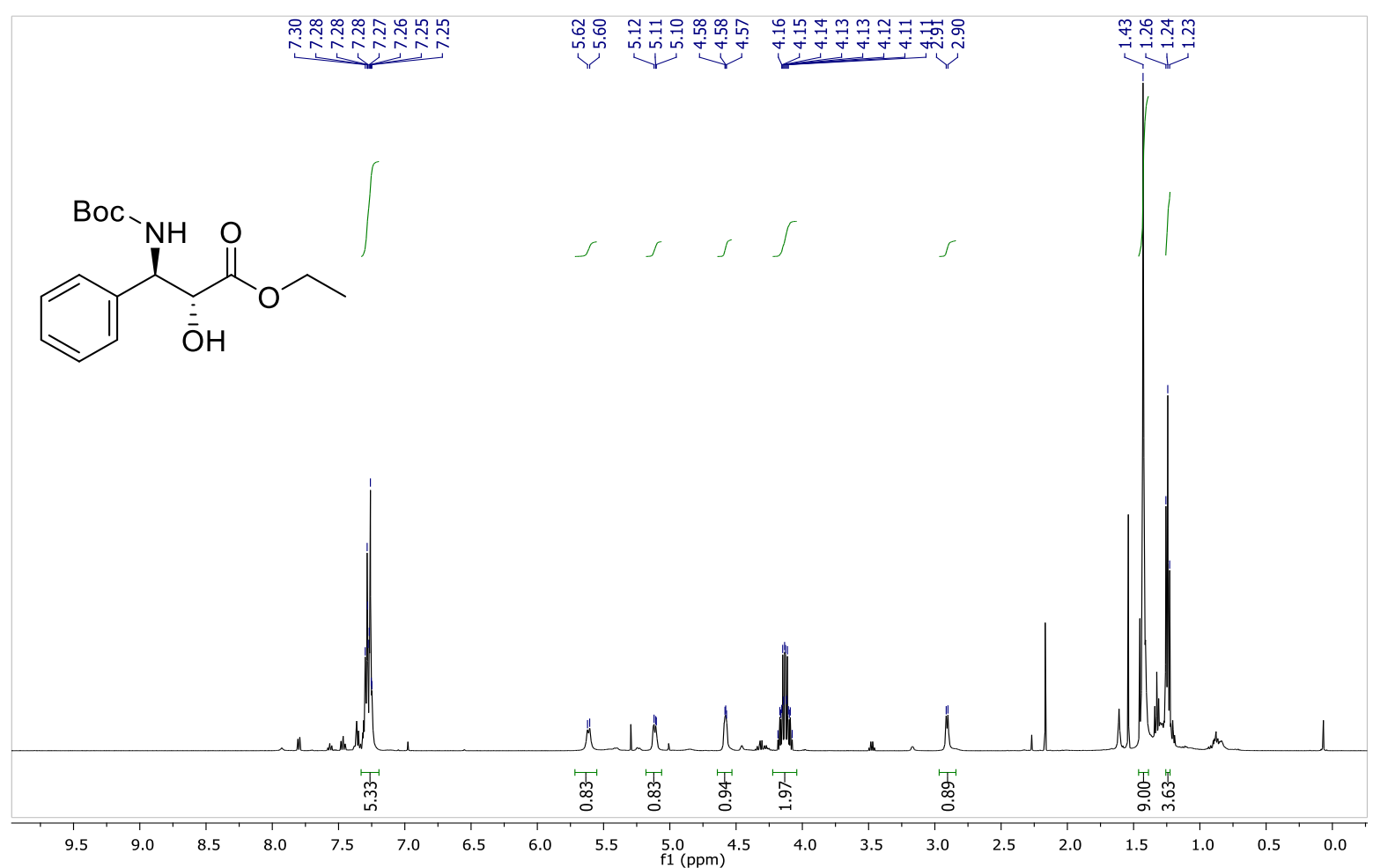

${ }^{13} \mathrm{C}\left\{{ }^{1} \mathrm{H}\right\}$ NMR spectrum of $\mathbf{5 b}(100 \mathrm{MHz})$

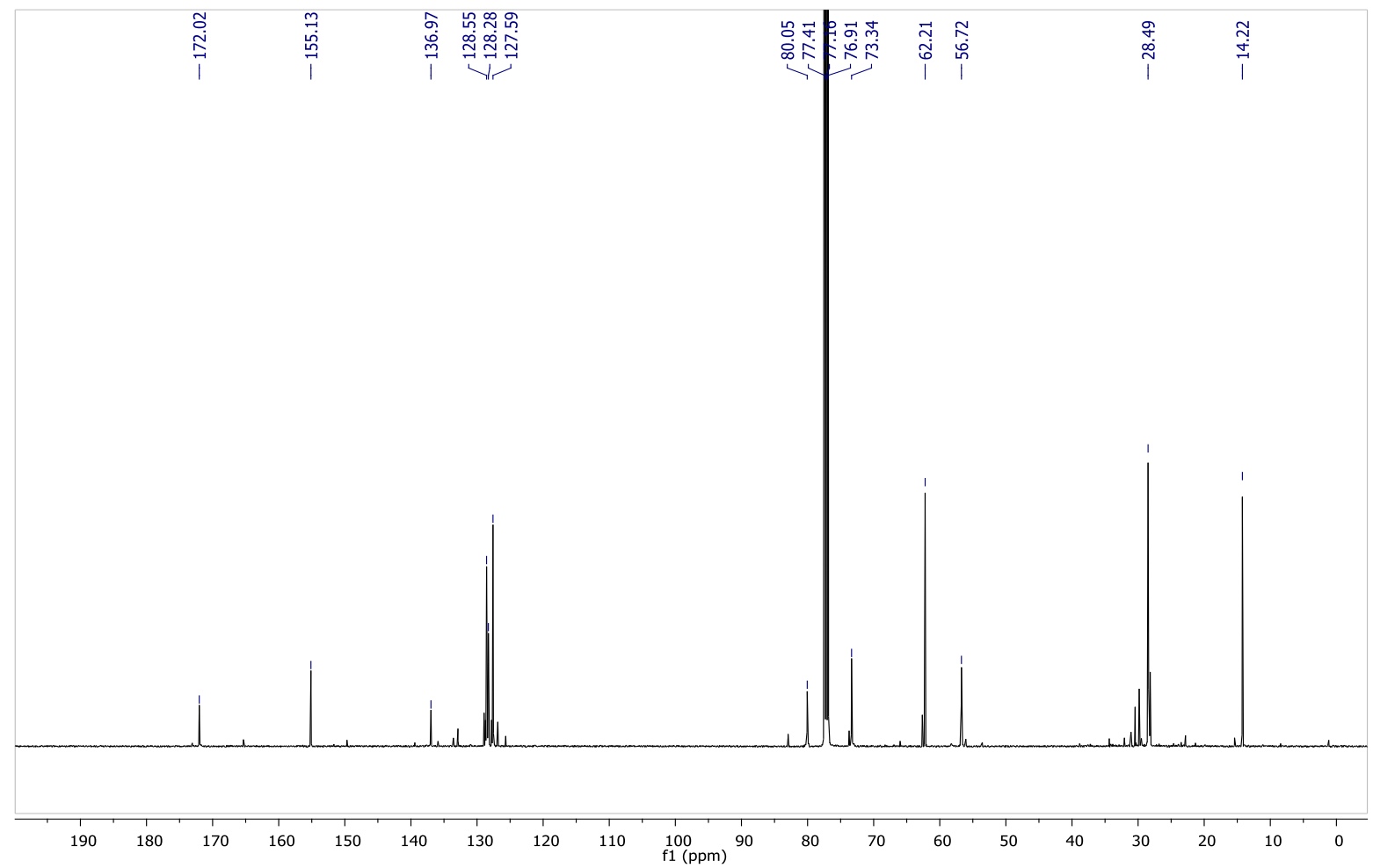


${ }^{1} \mathrm{H}-\mathrm{NMR}$ spectrum of $\mathbf{5 c}(500 \mathrm{MHz})$

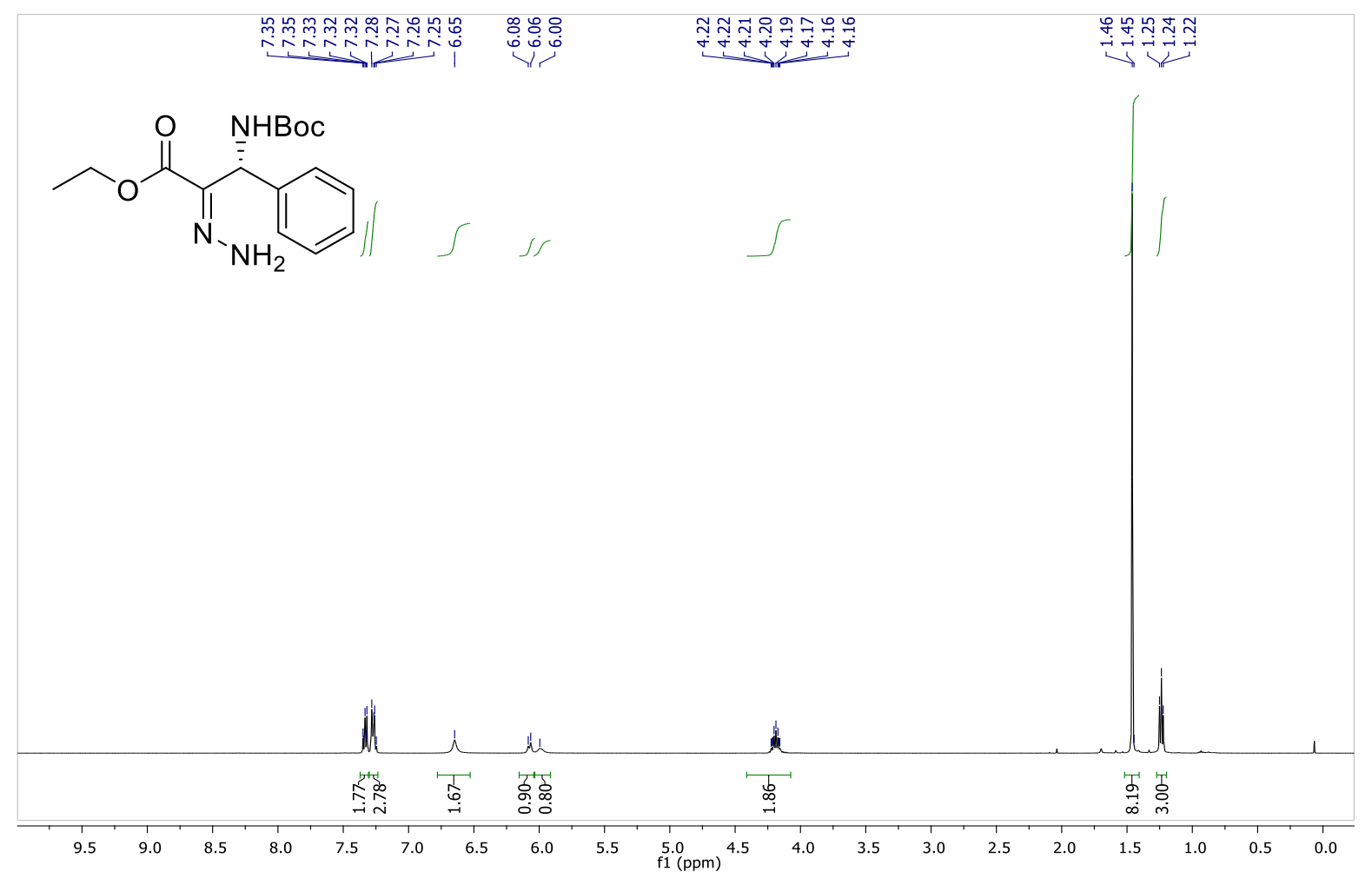

${ }^{13} \mathrm{C}\left\{{ }^{1} \mathrm{H}\right\}$ NMR spectrum of 5c $(125 \mathrm{MHz})$

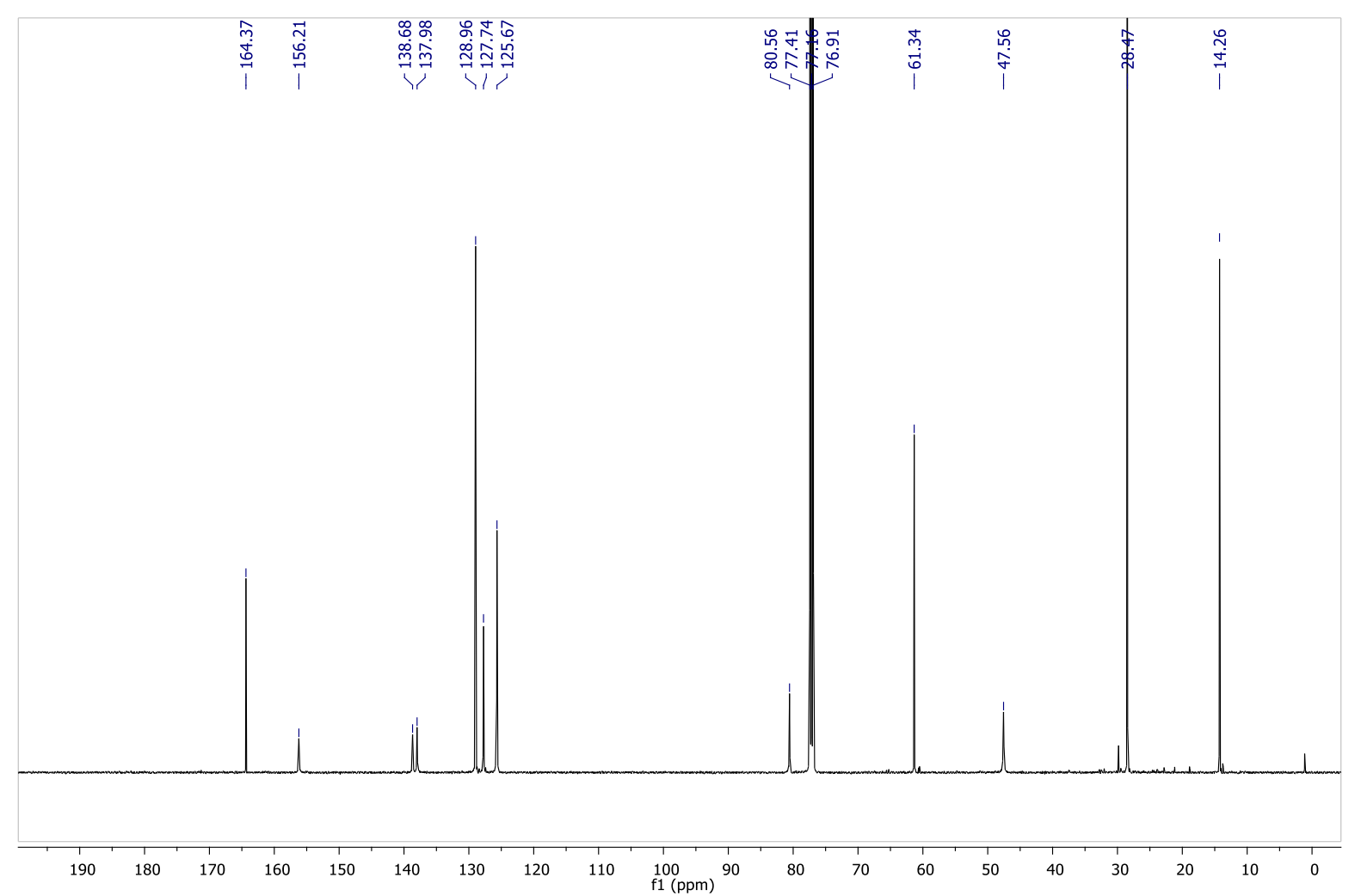


${ }^{1} \mathrm{H}-\mathrm{NMR}$ spectrum $5 \mathbf{d}(500 \mathrm{MHz})$

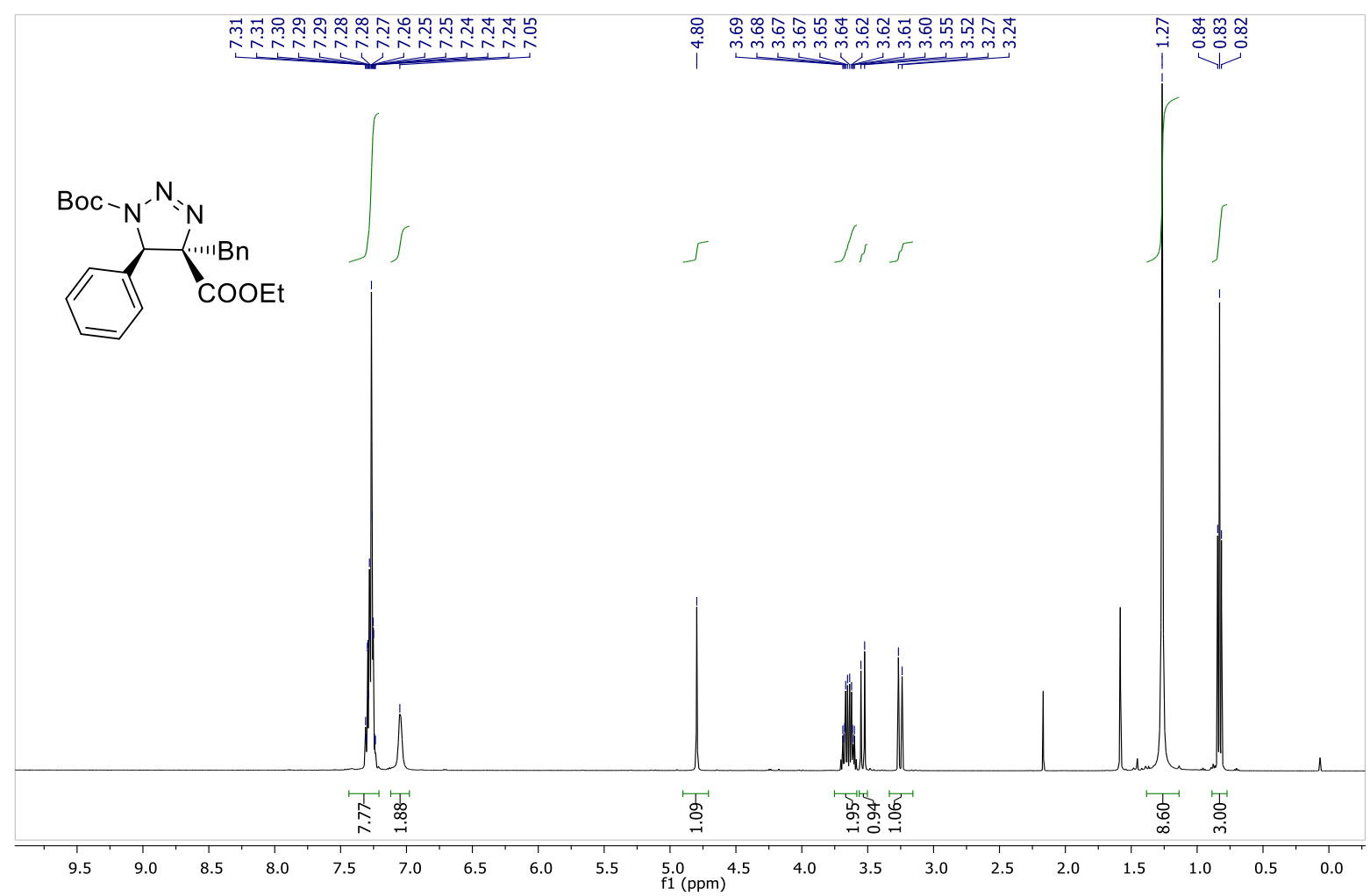

${ }^{13} \mathrm{C}\left\{{ }^{1} \mathrm{H}\right\}$ NMR spectrum of $\mathbf{5 d}(125 \mathrm{MHz})$

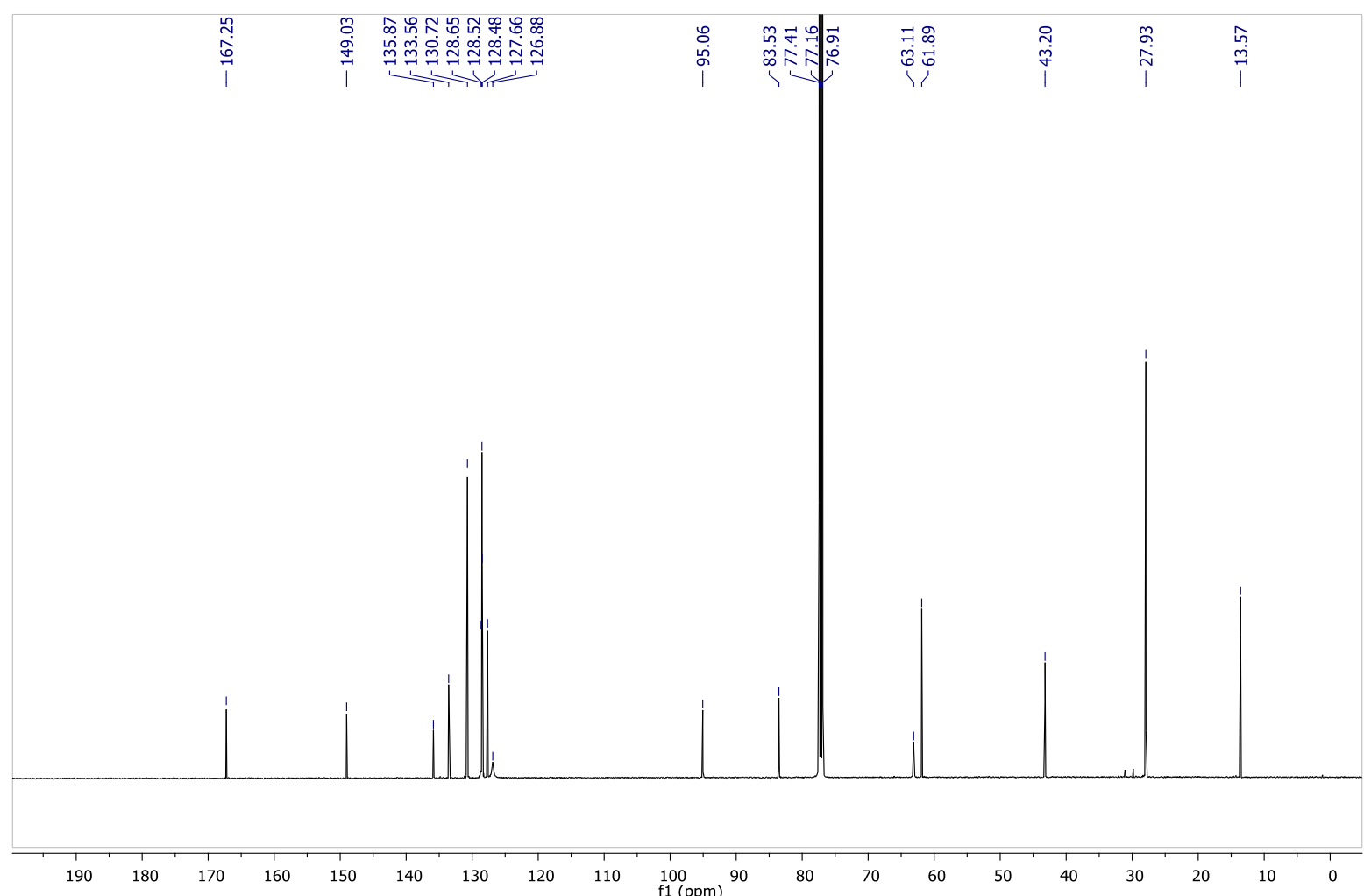




\subsection{Copies of HPLC spectra}

\section{HPLC chromatograms of $\mathbf{4 a}$}

Chiralpak AS-H, hexane/isopropanol = 95/5, $1 \mathrm{~mL} / \mathrm{min}$

Enantiomerically enriched ( $97 \%$ ee)

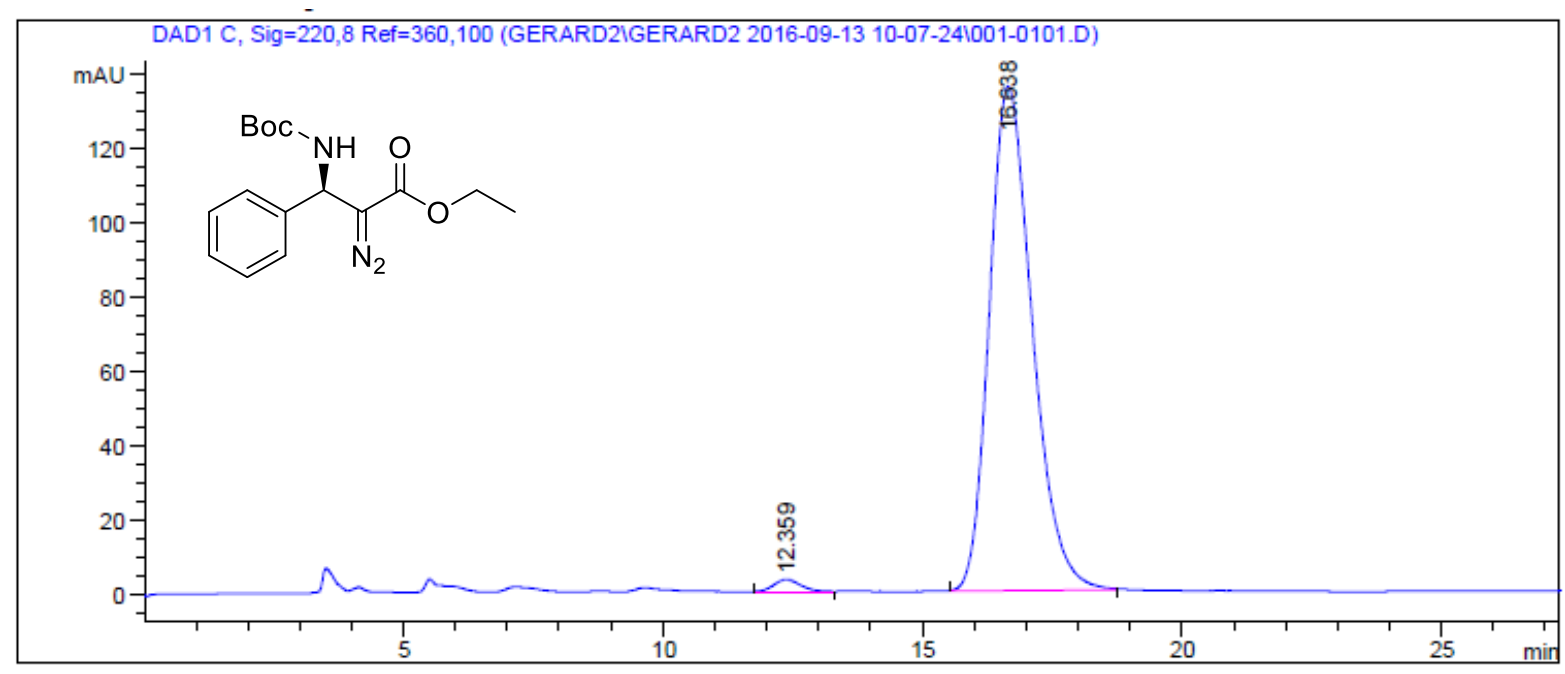

Signal 3: DAD1 C, Sig $=220,8$ Ref $=360,100$

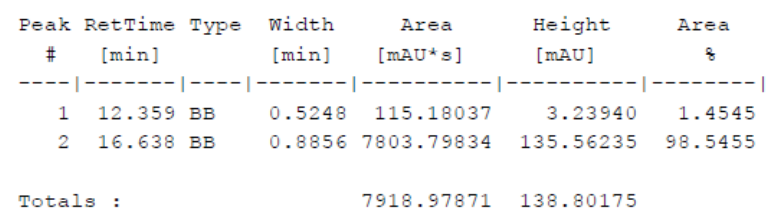

Racemic
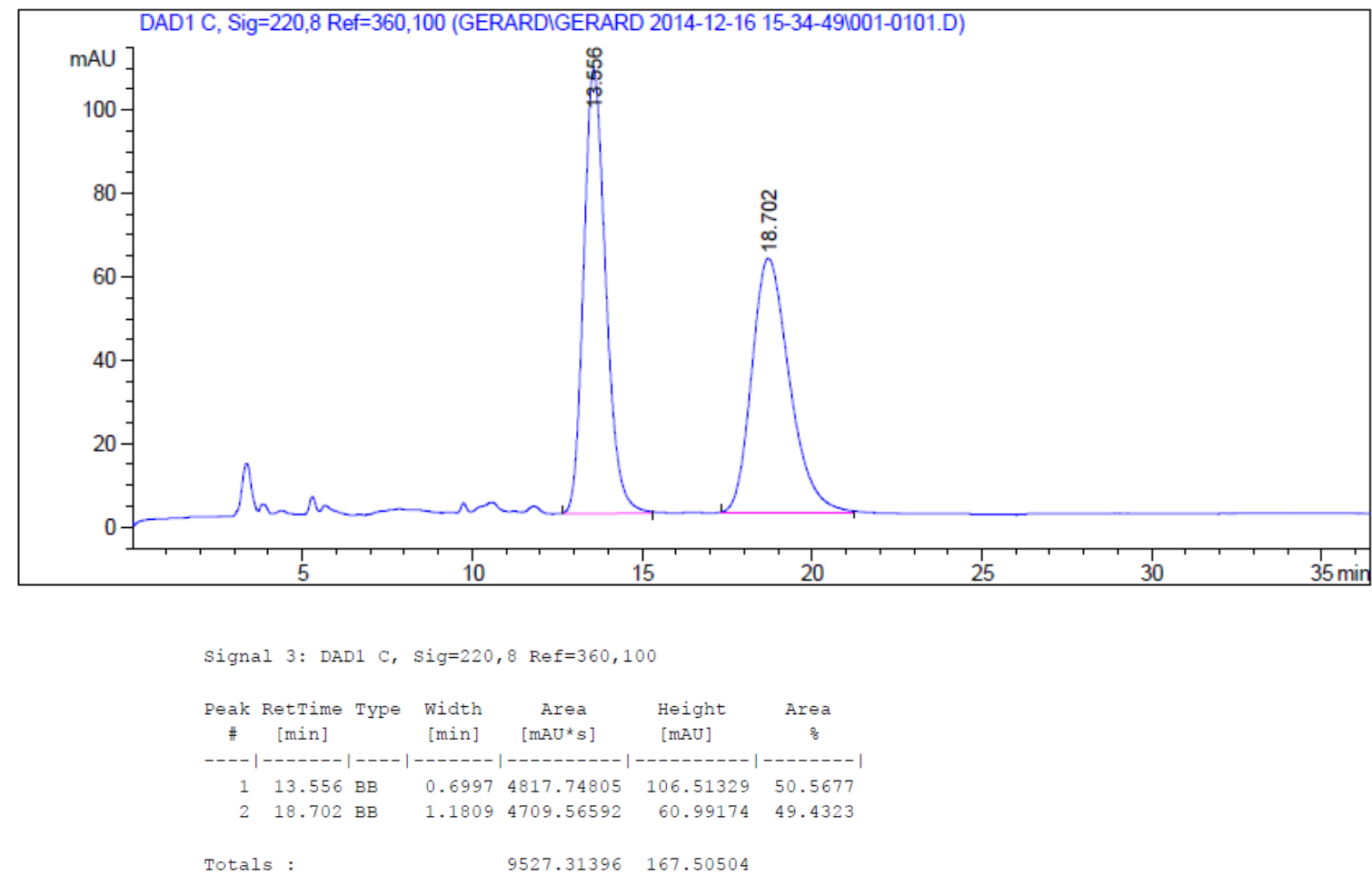


\section{HPLC chromatograms of $\mathbf{4 b}$}

Chiralpak AD-H, hexane/isopropanol = 95/5, $1 \mathrm{~mL} / \mathrm{min}$

\section{Enantiomerically enriched ( $98 \%$ ee)}

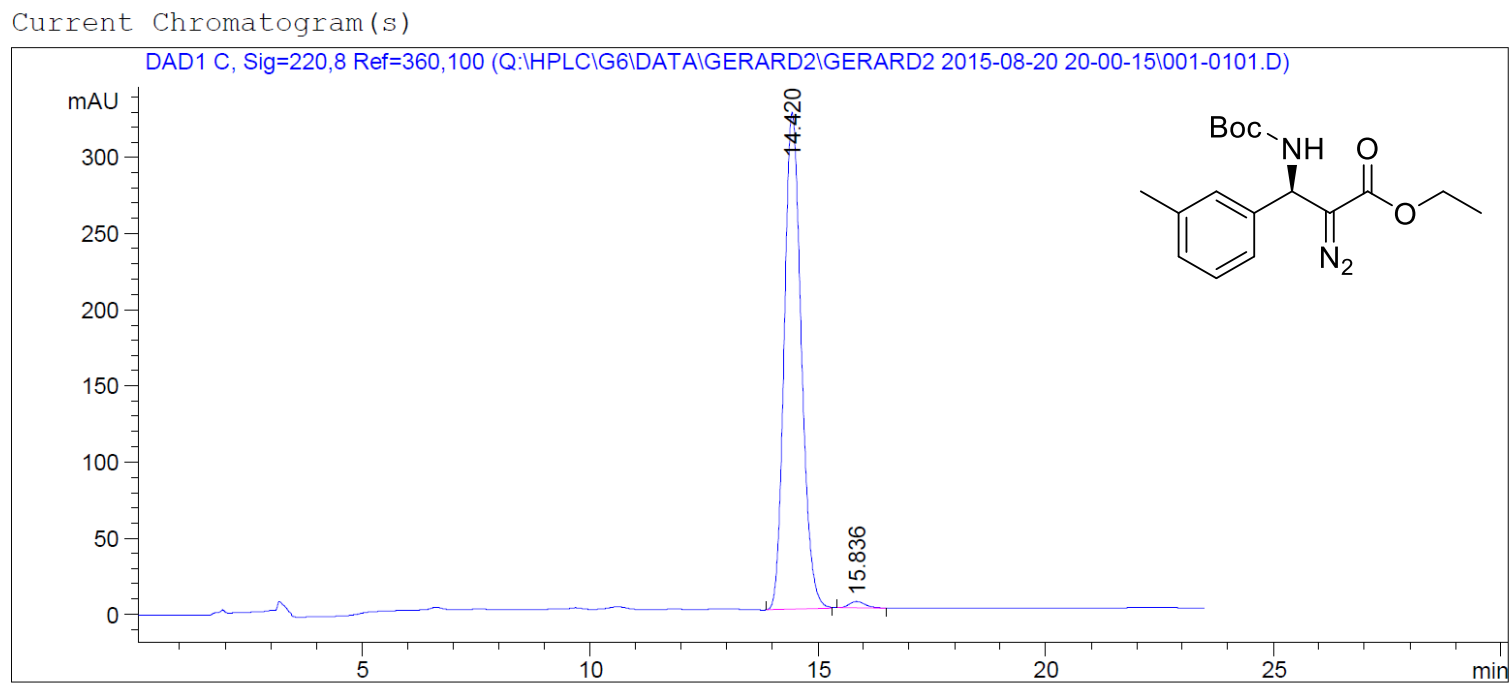

Signal 3: DAD1 C, Sig $=220,8$ Kef $=360,100$

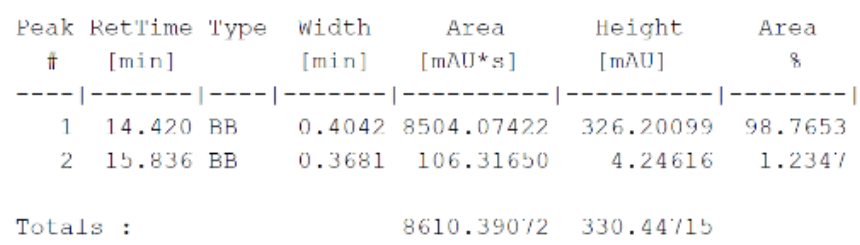

Racemic

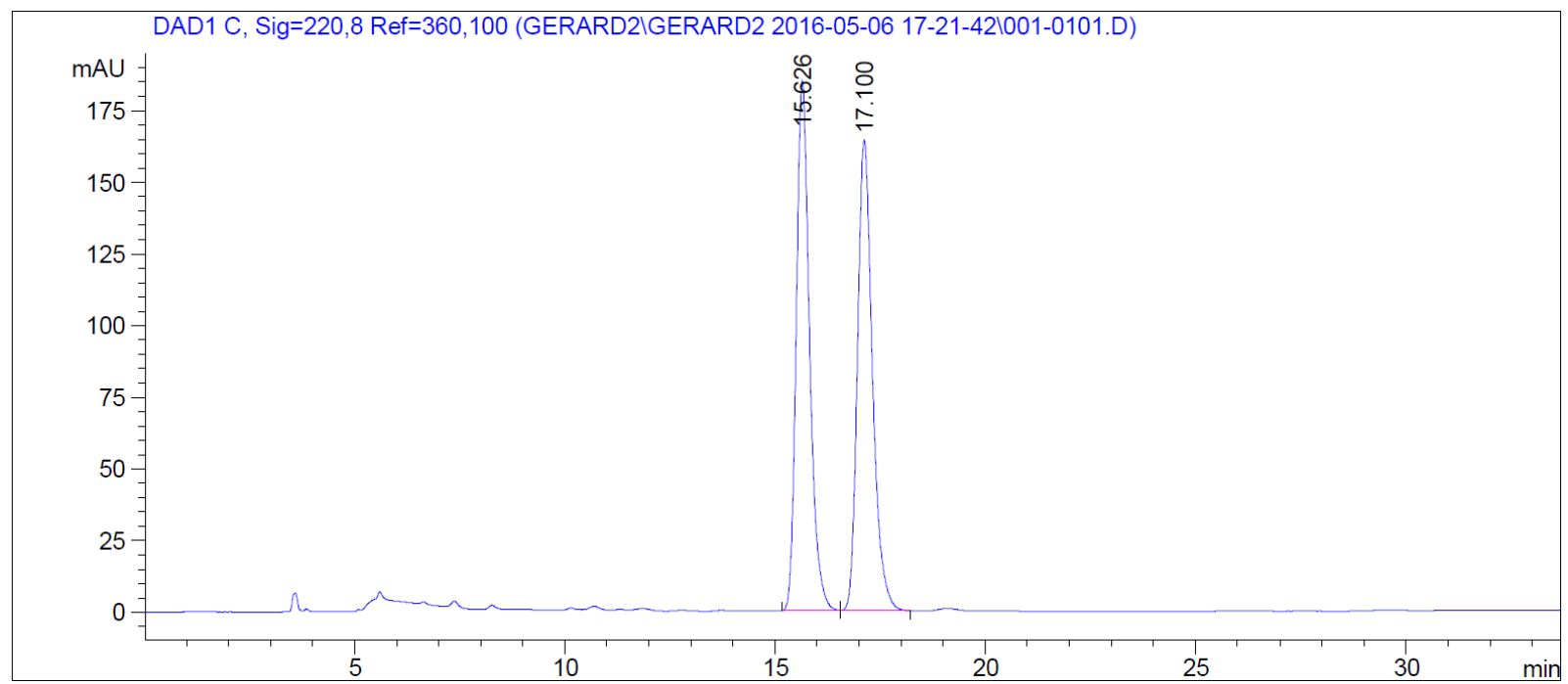

Signal 3: DAD1 C, $\operatorname{Sig}=220,8$ Ref $=360,100$

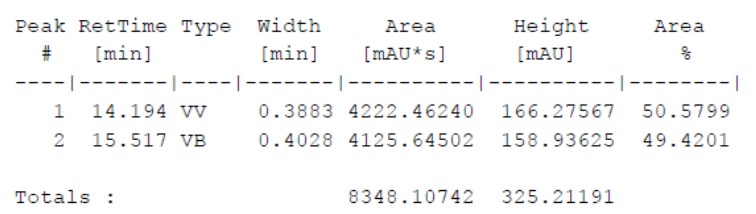




\section{HPLC chromatograms of $\mathbf{4 c}$}

Chiralpak AD-H, hexane/isopropanol = 95/5, $1 \mathrm{~mL} / \mathrm{min}$

Enantiomerically enriched ( $98 \%$ ee)

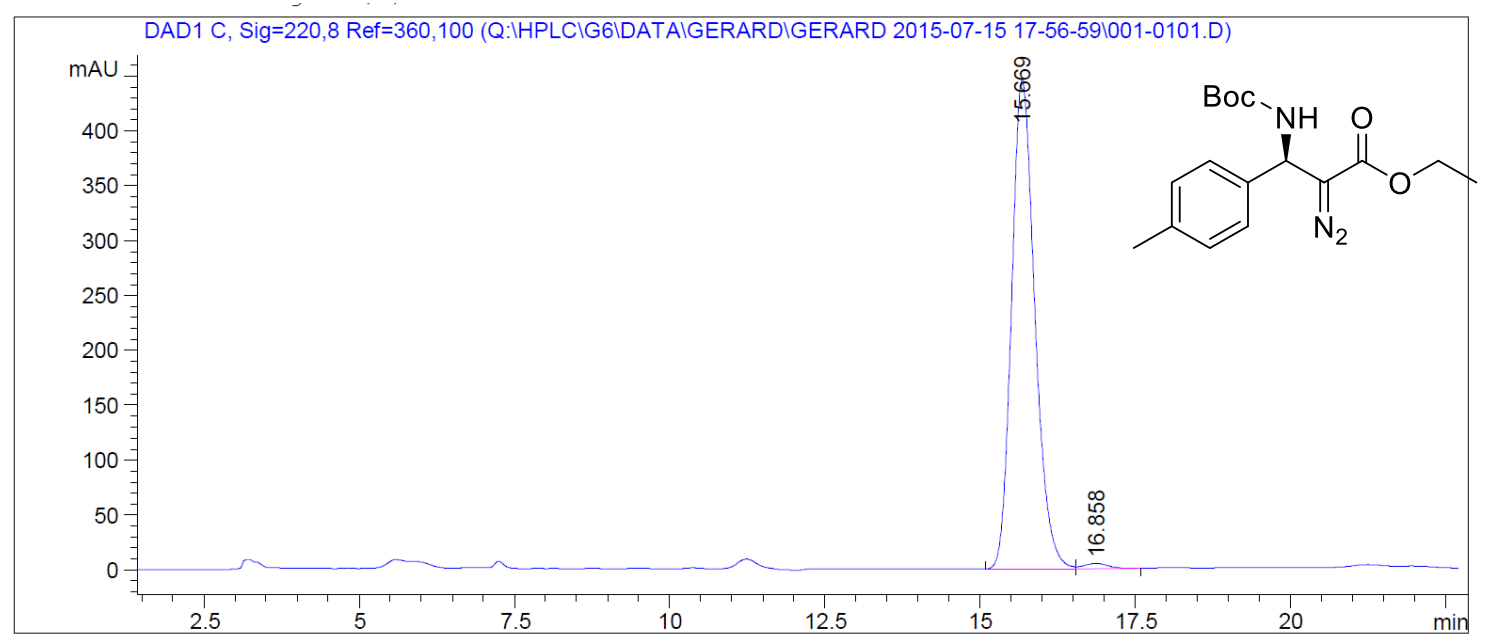

Signal 3: DAD1 C, $\operatorname{Sig}=220,8$ Ref $=360,100$

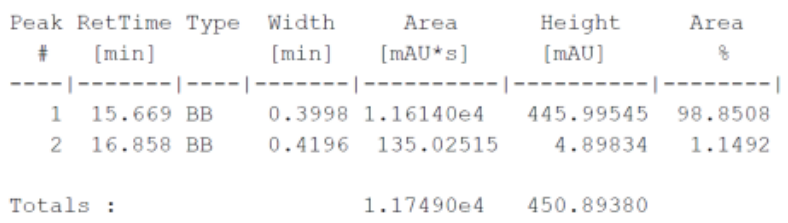

\section{Racemic}

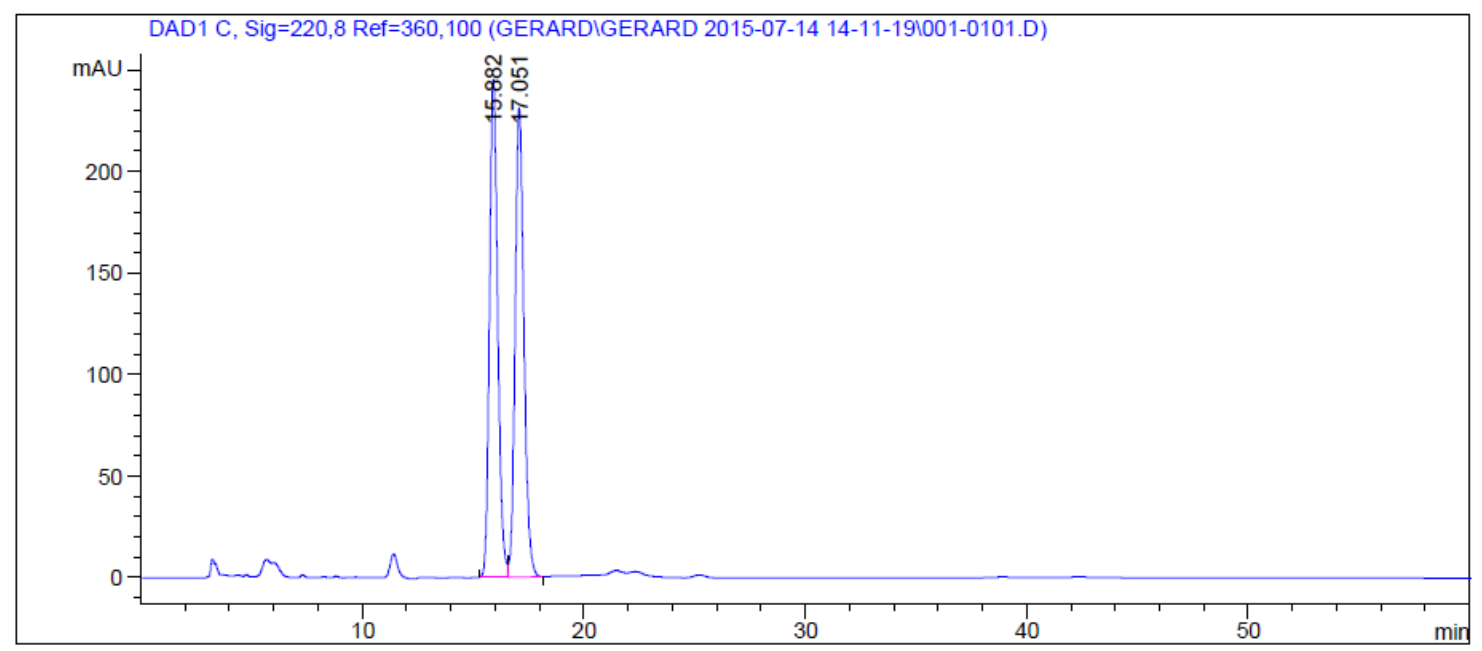

Signal 3: DAD1 C, Sig $=220,8$ Ref $=360,100$

\begin{tabular}{|c|c|c|c|c|c|c|}
\hline $\begin{array}{c}\text { Peak } \\
\#\end{array}$ & $\begin{array}{c}\text { RetTime } \\
{[\mathrm{min}]}\end{array}$ & Type & $\begin{array}{l}\text { Width } \\
\text { [min] }\end{array}$ & $\begin{array}{c}\text { Area } \\
{\left[\mathrm{mAU}^{*} \mathrm{~s}\right]}\end{array}$ & $\begin{array}{l}\text { Height } \\
{[\mathrm{mAU}]}\end{array}$ & $\begin{array}{c}\text { Area } \\
\frac{8}{8}\end{array}$ \\
\hline 1 & 15.882 & BV & 0.3981 & 6349.01318 & 245.20062 & 49.7954 \\
\hline 2 & 17.051 & VB & 0.4228 & 6401.18799 & 231.37578 & 50.2046 \\
\hline
\end{tabular}

Totals :

$1.27502 \mathrm{e} 4 \quad 476.57640$ 


\section{HPLC chromatograms of $\mathbf{4 d}$}

Chiralpak AD-H, hexane/isopropanol = 95/5, $1 \mathrm{~mL} / \mathrm{min}$

\section{Enantiomerically enriched ( $96 \%$ ee)}

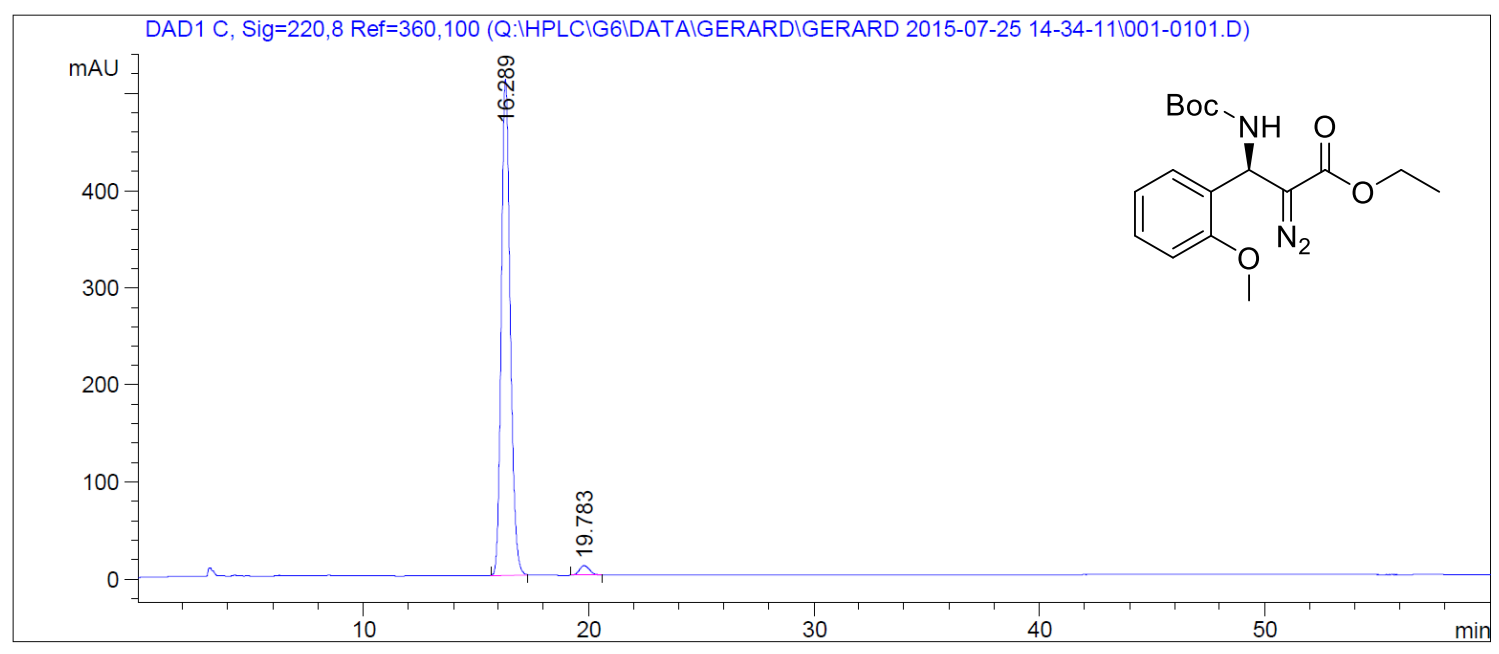

Signal 3: DAD1 C, Sig=220,8 Ref $=360,100$

\begin{tabular}{|c|c|c|c|c|c|c|}
\hline $\begin{array}{c}\text { Peak } \\
\quad \#\end{array}$ & $\begin{array}{c}\text { Retrime } \\
\text { [min] }\end{array}$ & Type & $\begin{array}{c}\text { Width } \\
\text { [min] }\end{array}$ & $\begin{array}{c}\text { Area } \\
{\left[\mathrm{mAU}^{*} \mathrm{~s}\right]}\end{array}$ & $\begin{array}{l}\text { Height } \\
\text { [MAU] }\end{array}$ & $\begin{array}{c}\text { Area } \\
\quad \frac{8}{8}\end{array}$ \\
\hline & & & --- & -- & ---- & ------ \\
\hline 1 & 16.289 & $\mathrm{BB}$ & 0.4264 & $1.41172 \mathrm{e} 4$ & 510.82791 & 97.8068 \\
\hline 2 & 19.783 & $\mathrm{BB}$ & 0.4862 & 316.56369 & 10.03116 & 2.1932 \\
\hline otal & $\mathrm{s}:$ & & & $1.44338 \mathrm{e} 4$ & 520.85907 & \\
\hline
\end{tabular}

\section{Racemic}

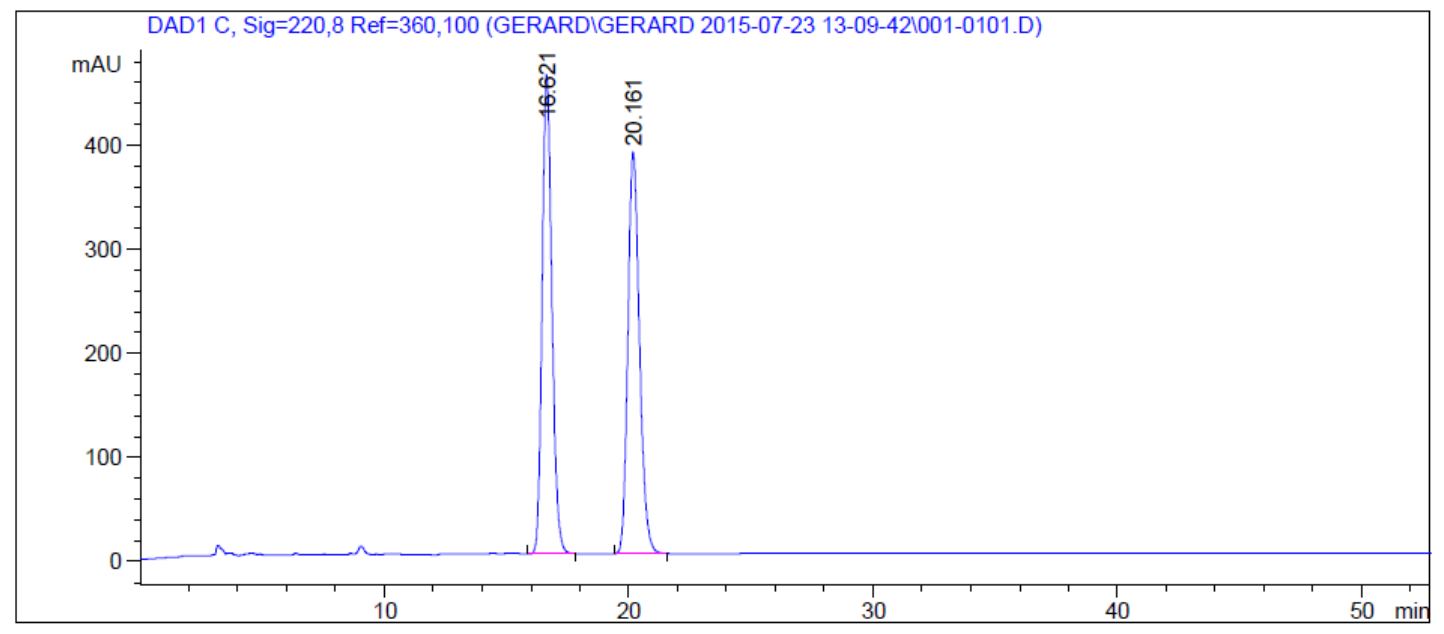

Signal 3: DAD1 C, Sig $=220,8$ Ref $=360,100$

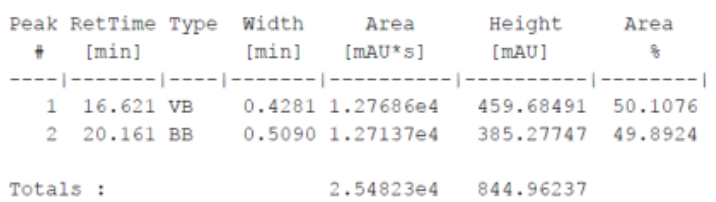




\section{HPLC chromatograms of $\mathbf{4 e}$}

Chiralpak AD-H, hexane/isopropanol = 95/5, $1 \mathrm{~mL} / \mathrm{min}$

\section{Enantiomerically enriched ( $97 \%$ ee)}

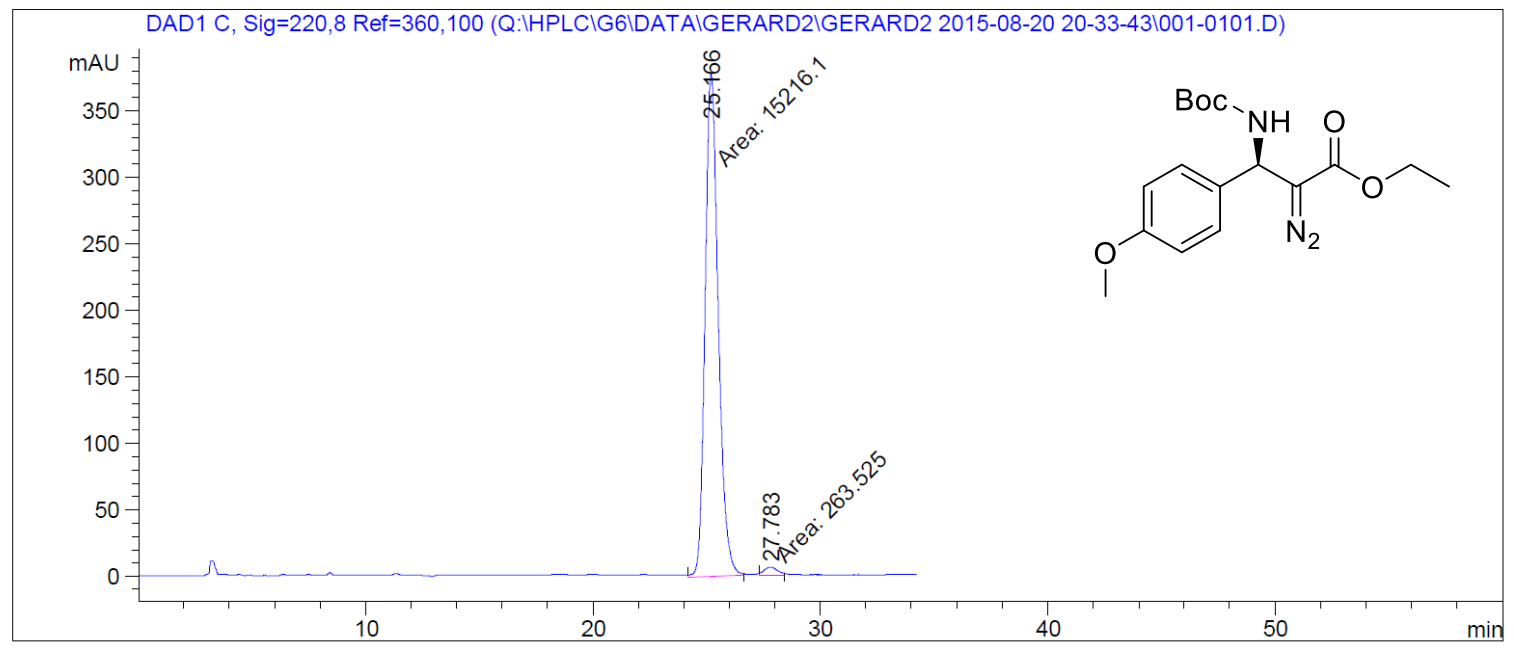

Signal 3: DAD1 C, Sig-220,8 Ref-360,100

\begin{tabular}{|c|c|c|c|c|c|c|}
\hline $\begin{array}{c}\text { Peak } \\
\text { \# }\end{array}$ & $\begin{array}{c}\text { RelTime } \\
\text { [min] }\end{array}$ & Type & $\begin{array}{l}\text { midalh } \\
\text { [min] }\end{array}$ & $\begin{array}{c}\text { Ared } \\
{\left[\mathrm{m} \lambda U^{*} \mathrm{~s}\right]}\end{array}$ & $\begin{array}{l}\text { Height } \\
{[\mathrm{m} \lambda \mathrm{U}]}\end{array}$ & $\begin{array}{c}\text { Area } \\
8\end{array}$ \\
\hline & & & & 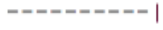 & ---1 & -- \\
\hline 1 & 25.16 & M & 0.6713 & $1.52161 \mathrm{c} 4$ & 377.76056 & 2976 \\
\hline 2 & 27.783 & MM & 0.7033 & 263.52539 & 6.24466 & 1.7024 \\
\hline
\end{tabular}

Totals : $\quad 1.54796 e 4 \quad 384.00522$

\section{Racemic}

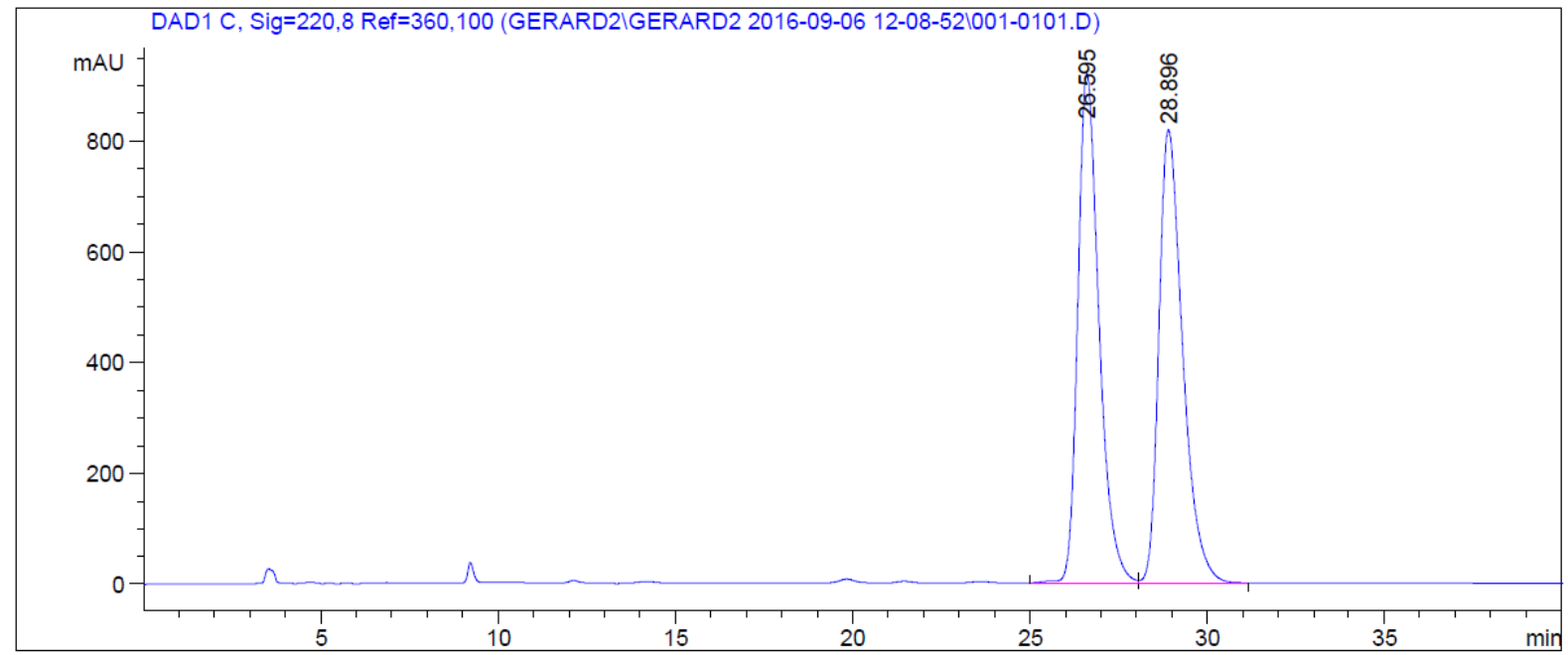

Signal 3: DAD1 C, Sig=220,8 Ref $=360,100$

\begin{tabular}{|c|c|c|c|c|c|c|}
\hline $\begin{array}{c}\text { Peak } \\
\#\end{array}$ & $\begin{array}{c}\text { RetTime } \\
\text { [min] }\end{array}$ & Type & $\begin{array}{l}\text { Width } \\
{[\min ]}\end{array}$ & $\begin{array}{c}\text { Area } \\
{\left[\mathrm{mAU}^{\star} \mathrm{s}\right]}\end{array}$ & $\begin{array}{l}\text { Height } \\
\text { [mAU] }\end{array}$ & $\begin{array}{c}\text { Area } \\
\frac{8}{8}\end{array}$ \\
\hline 1 & 26.595 & BV & 0.6333 & $3.82698 e 4$ & 920.02899 & 50.0896 \\
\hline 2 & 28.896 & VB & 0.7113 & $3.81329 e 4$ & 818.79401 & 49.9104 \\
\hline
\end{tabular}




\section{HPLC chromatograms of $\mathbf{4 f}$}

Chiralpak AD-H, hexane/isopropanol = 95/5, $1 \mathrm{~mL} / \mathrm{min}$

Enantiomerically enriched ( $96 \%$ ee)

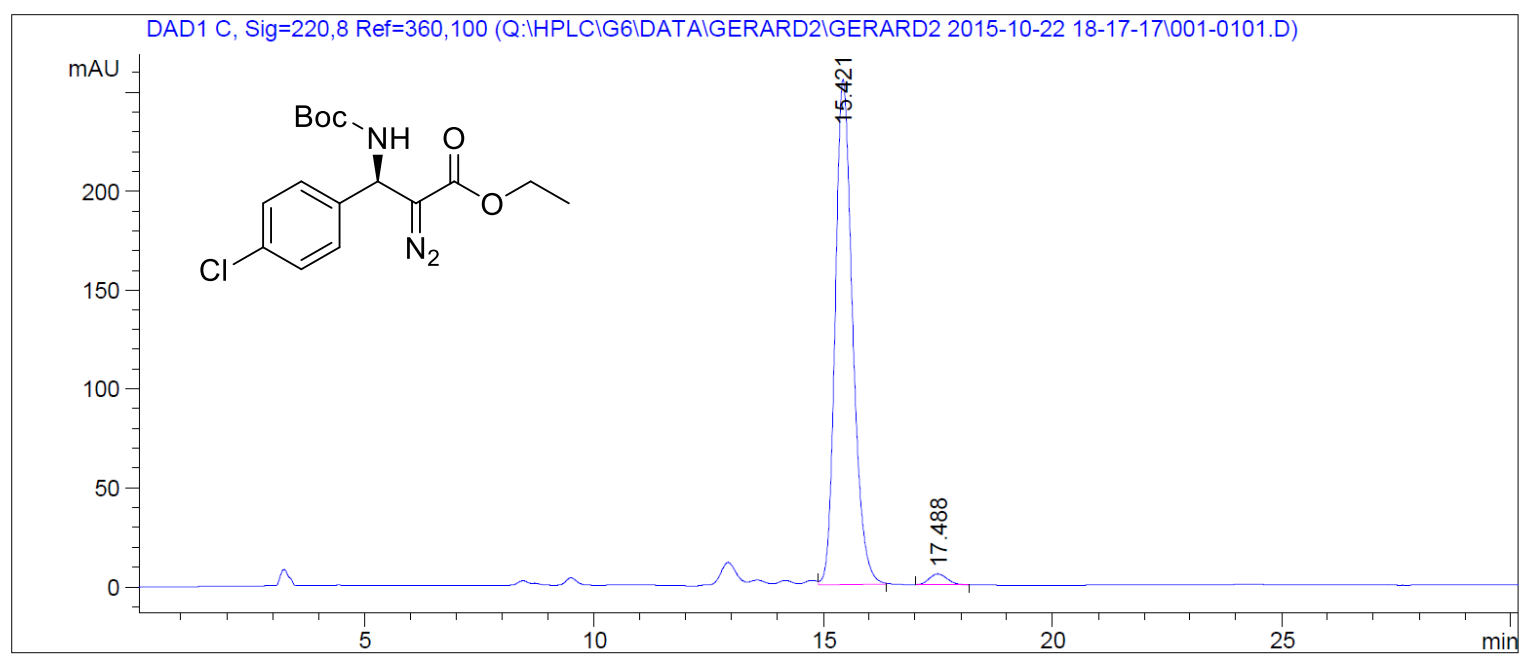

Signal 3: DAD1 C, Sig $=220,8$ Ref $=360,100$

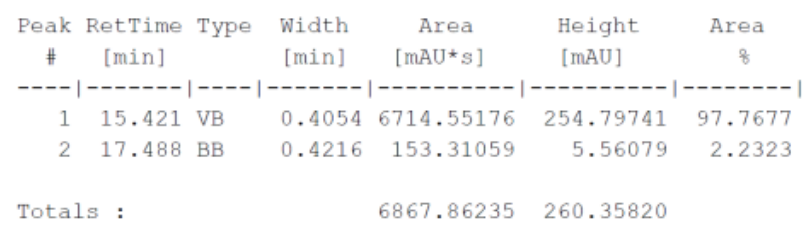

\section{Racemic}

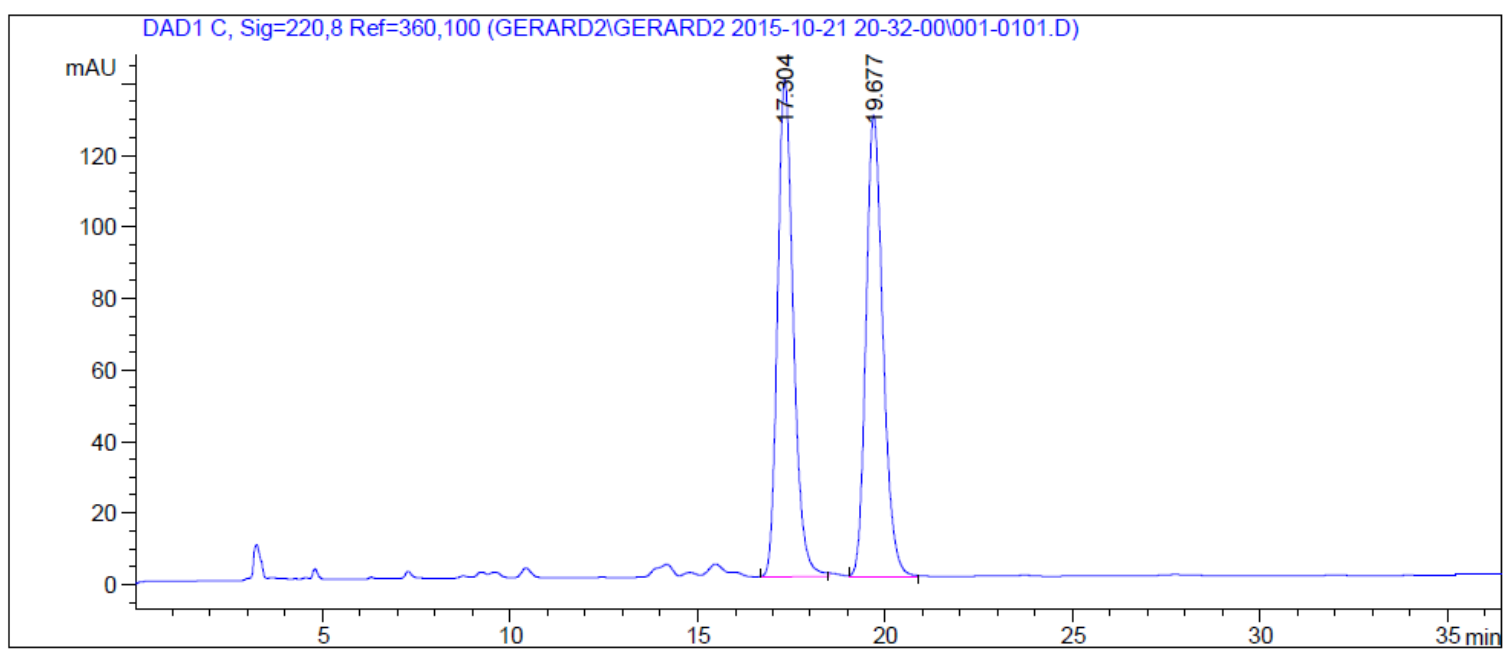

Signal 3: DAD1 C, $\operatorname{Sig}=220,8$ Ref $=360,100$

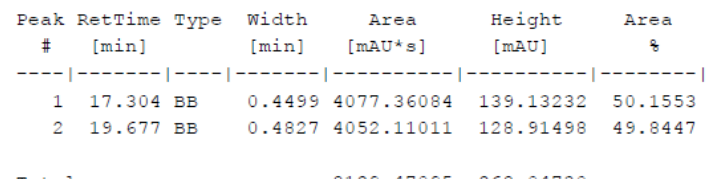

Totals : 


\section{HPLC chromatograms of $\mathbf{4 g}$}

Chiralpak AD-H, hexane/isopropanol = 95/5, $1 \mathrm{~mL} / \mathrm{min}$

\section{Enantiomerically enriched ( $94 \%$ ee)}

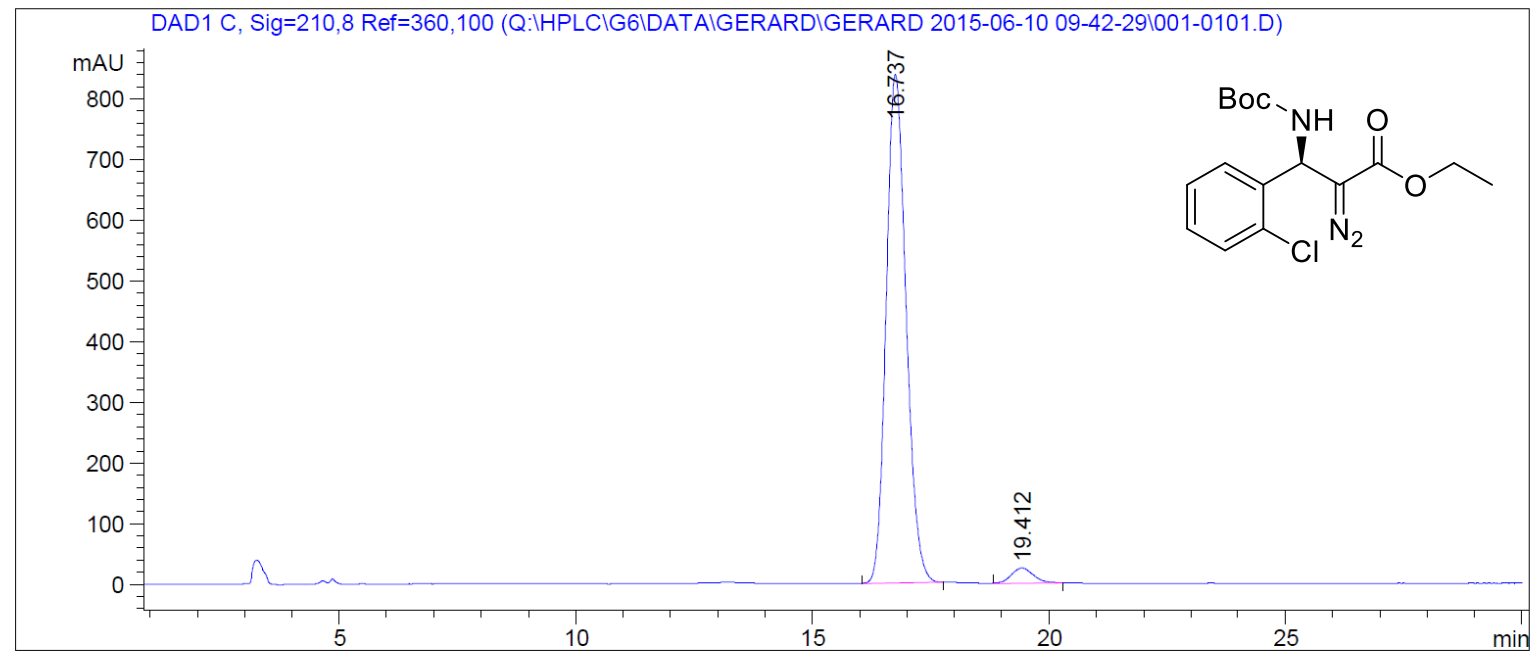

Signal 3: DAD1 C, Sig $=210,8$ Ref $=360,100$

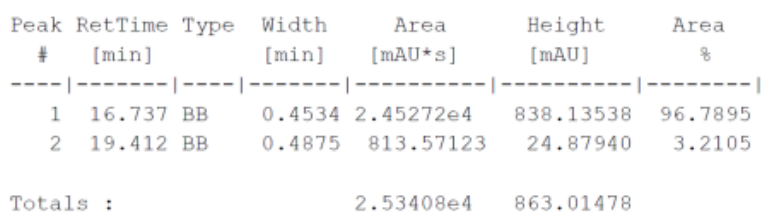

\section{Racemic}

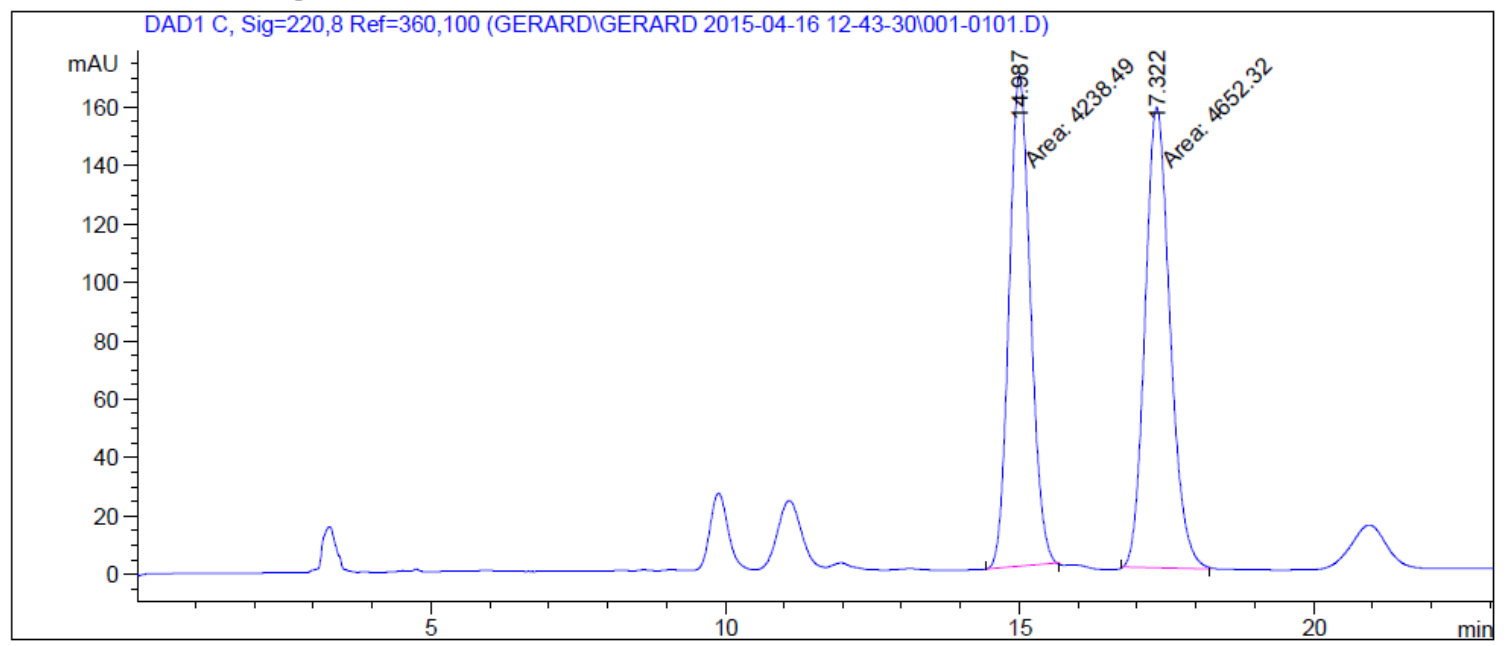

Signal 3: DAD1 C, $\operatorname{Sig}=220,8$ Ref $=360,100$

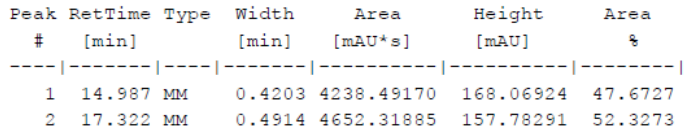

nstrument 1 1/11/2016 7:39 pm Jonny 


\section{HPLC chromatograms of $\mathbf{4 h}$}

Chiralpak AD-H, hexane/isopropanol = 95/5, $1 \mathrm{~mL} / \mathrm{min}$

Enantiomerically enriched ( $96 \%$ ee)

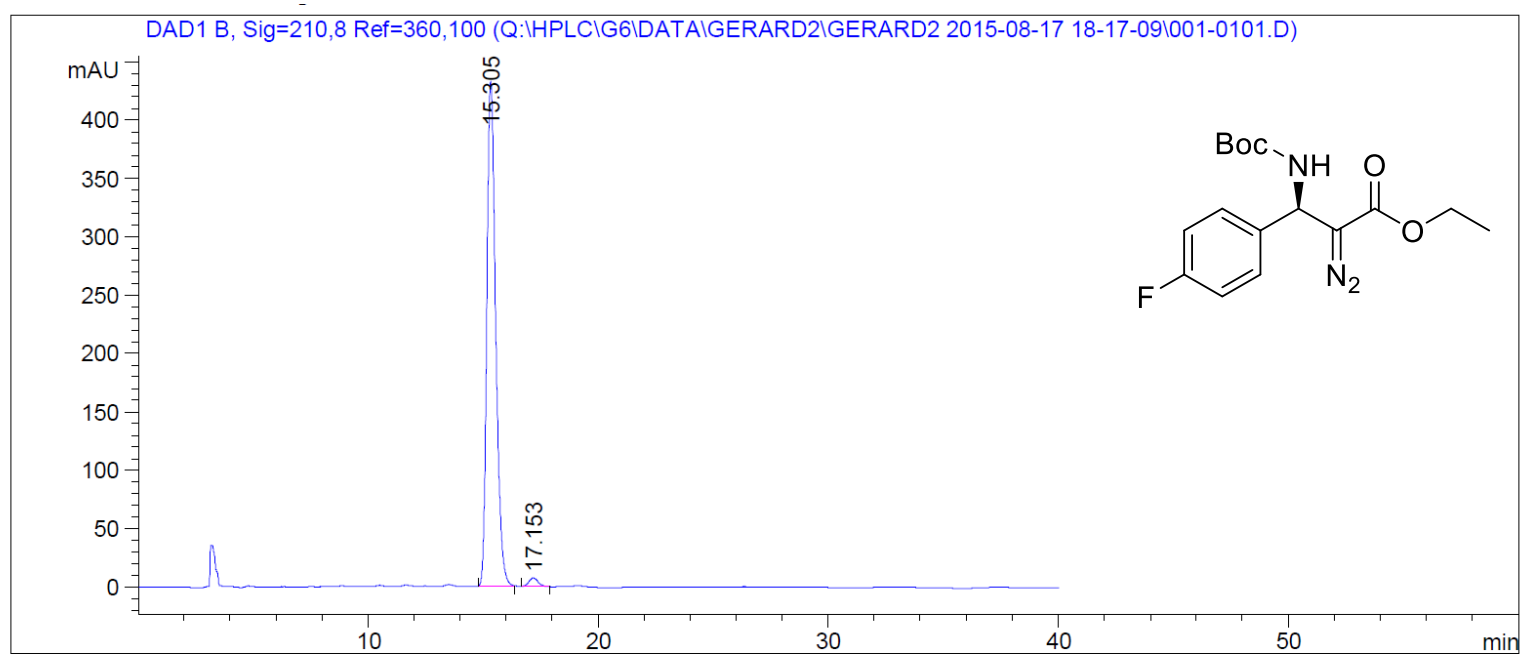

Signal 3: DAD1 C, Sig-220,8 Ref-360,100

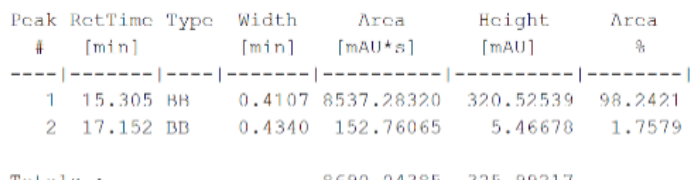

Tolaly :

$8690.04385 \quad 325.99217$

\section{Racemic}

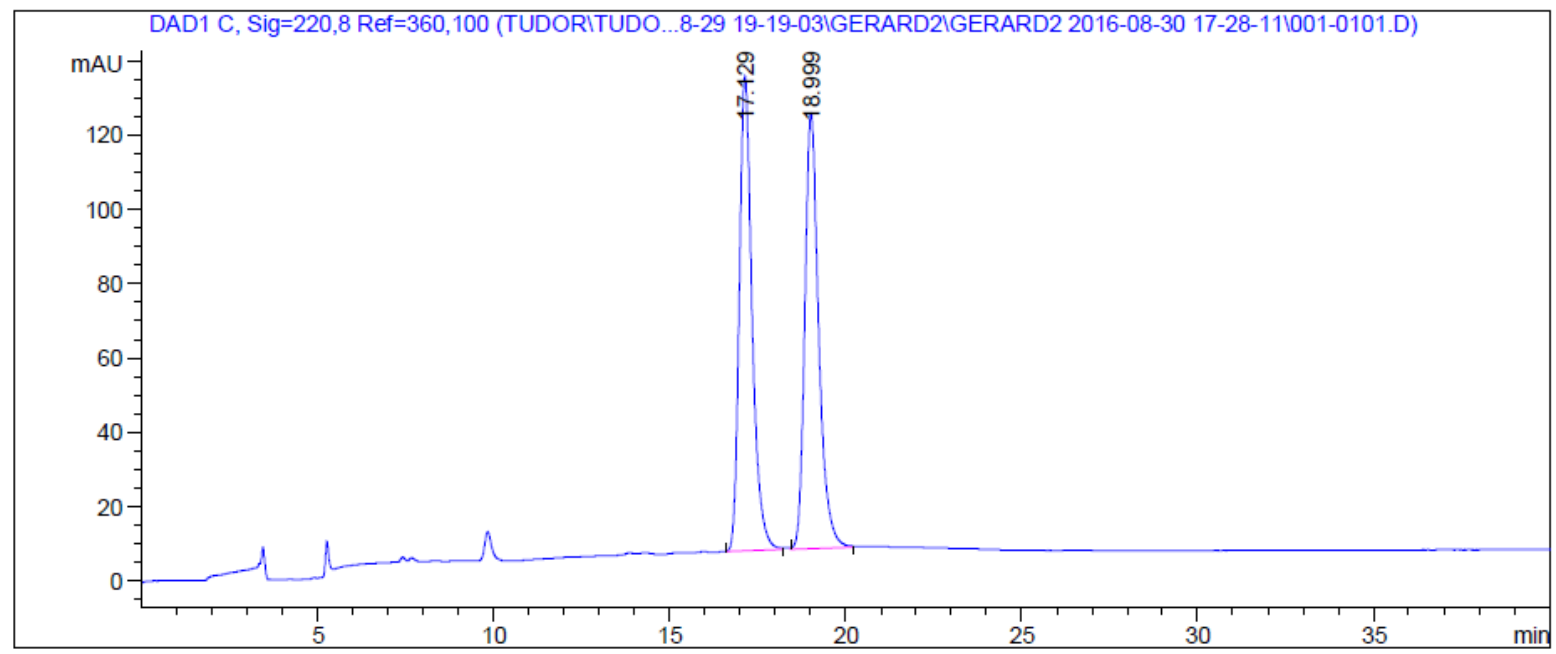

Signal 3: DAD1 C, $\operatorname{Sig}=220,8$ Ref $=360,100$

\begin{tabular}{|c|c|c|c|c|c|c|}
\hline $\begin{array}{c}\text { Peak } \\
\#\end{array}$ & $\begin{array}{l}\text { RetTime } \\
\text { [min] }\end{array}$ & Type & $\begin{array}{l}\text { Width } \\
\text { [min] }\end{array}$ & $\begin{array}{c}\text { Area } \\
{\left[\mathrm{mAU}^{*} \mathrm{~s}\right]}\end{array}$ & $\begin{array}{l}\text { Height } \\
\text { [mAU] }\end{array}$ & $\begin{array}{c}\text { Area } \\
\text { \& }\end{array}$ \\
\hline 1 & 17.129 & BB & 0.3817 & 3220.39551 & 127.92717 & 49.8314 \\
\hline 2 & 18.999 & BB & 0.4191 & 3242.18237 & 117.07170 & 50.1686 \\
\hline Tot & Is : & & & 6462.57788 & 244.99887 & \\
\hline
\end{tabular}




\section{HPLC chromatograms of $\mathbf{4 i}$}

Chiralpak AS-H, hexane/isopropanol = 95/5, $1 \mathrm{~mL} / \mathrm{min}$

Enantiomerically enriched (95\% ee)

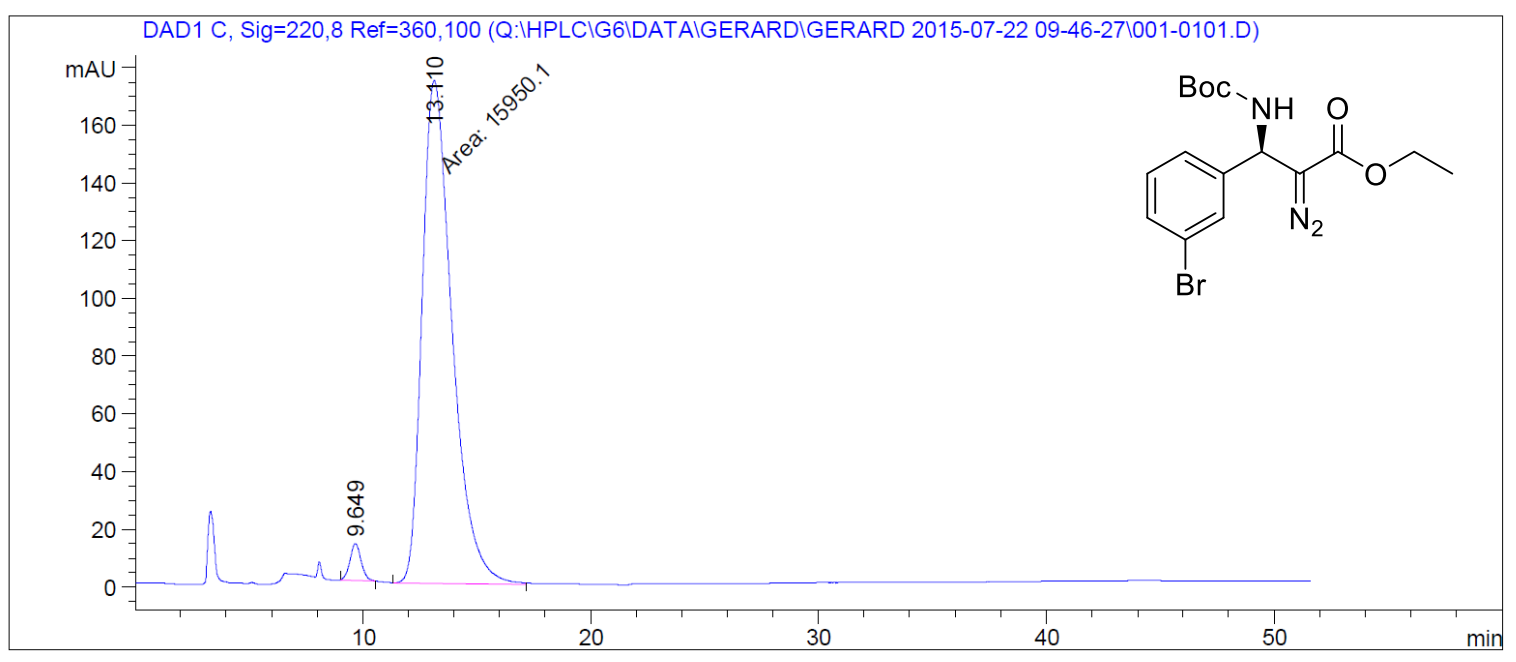

Signal 3: DAD1 C, Sig=220,8 Ref $=360,100$

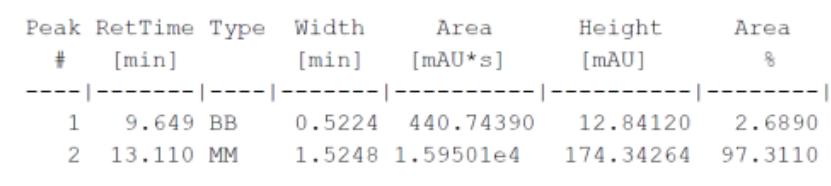

Totals : $\quad 1.63908 e 4 \quad 187.18383$

\section{Racemic}

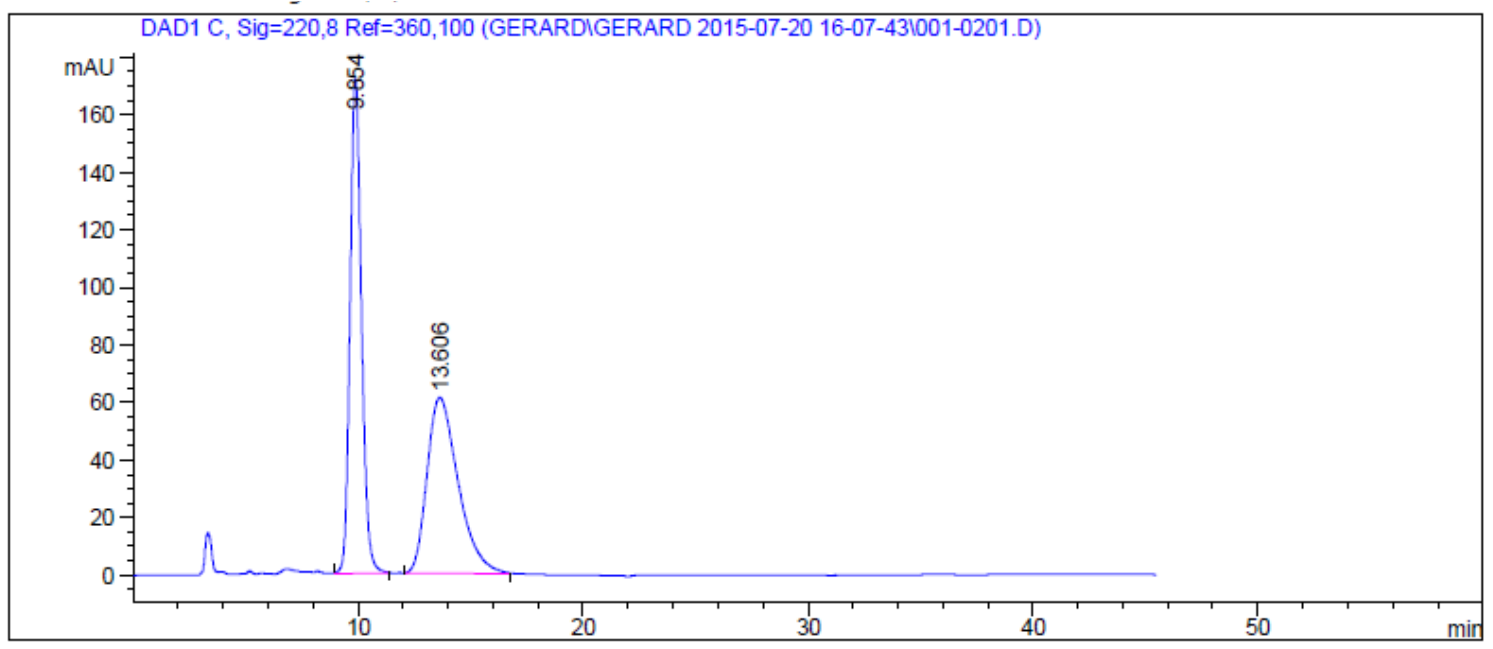

Signal 3: DAD1 C, Sig=220,8 Ref $=360,100$

\begin{tabular}{|c|c|c|c|c|c|c|}
\hline $\begin{array}{c}\text { Peak } \\
\#\end{array}$ & $\begin{array}{l}\text { RetTime } \\
\text { [min] }\end{array}$ & Type & $\begin{array}{l}\text { Width } \\
\text { [min] }\end{array}$ & $\begin{array}{c}\text { Area } \\
{\left[\mathrm{mAU}^{\star} \mathrm{s}\right]}\end{array}$ & $\begin{array}{l}\text { Height } \\
\text { [mAU] }\end{array}$ & $\begin{array}{c}\text { Area } \\
\frac{8}{8}\end{array}$ \\
\hline -1. & 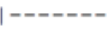 & & 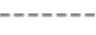 & 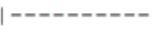 & ------- & ---- \\
\hline 1 & 9.854 & BB & 0.5435 & 6067.53564 & 172.01314 & 50.8498 \\
\hline 2 & 13.606 & $\mathrm{BB}$ & 1.4626 & 5864.72656 & 61.05613 & 49.1502 \\
\hline Tot & : & & & $1.19323 \mathrm{e} 4$ & 233.06927 & \\
\hline
\end{tabular}




\section{HPLC chromatograms of $\mathbf{4} \mathbf{j}$}

Chiralpak AS-H, hexane/isopropanol = 95/5, $1 \mathrm{~mL} / \mathrm{min}$

Enantiomerically enriched (98\%ee)
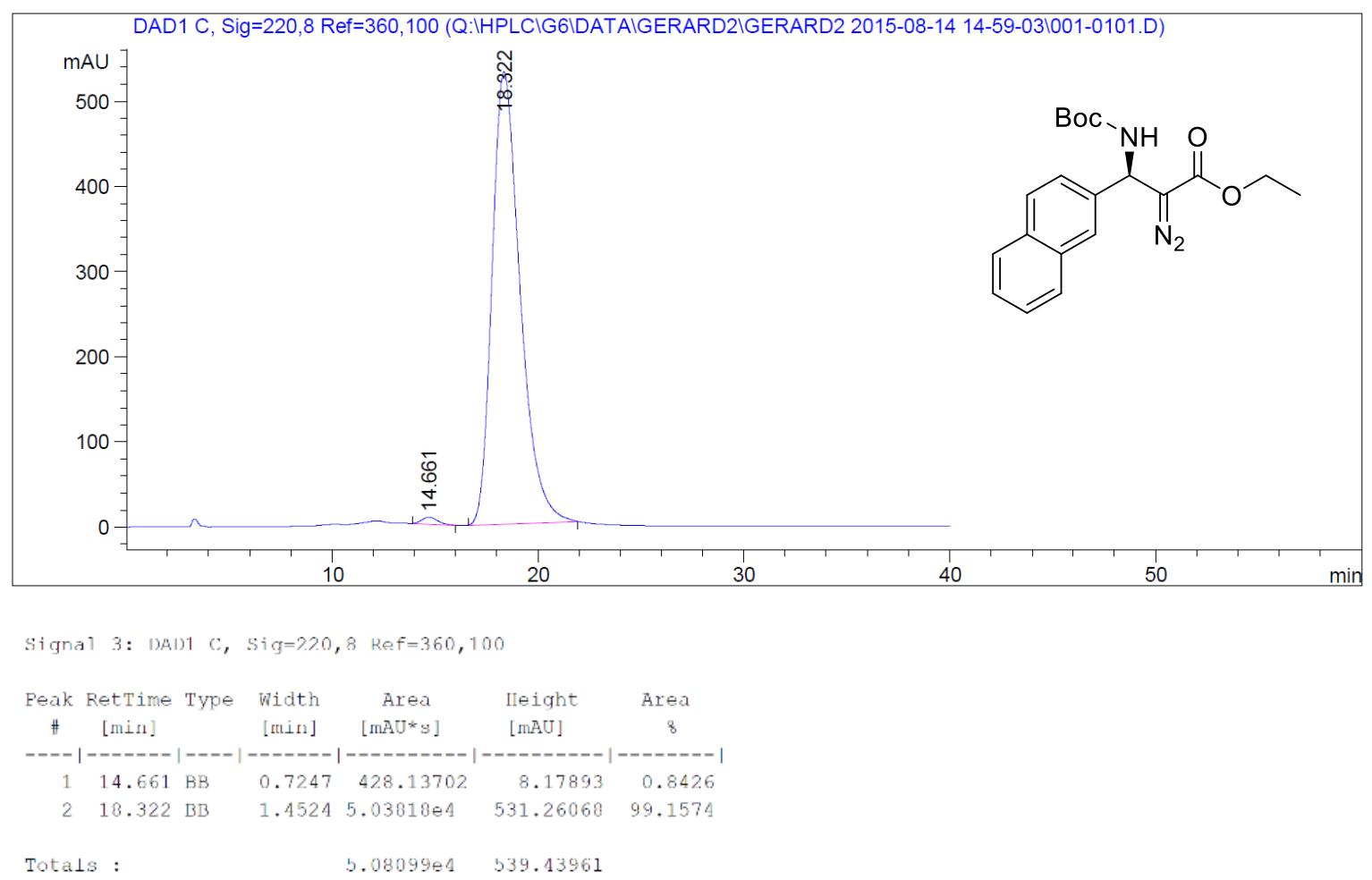

\section{Racemic}

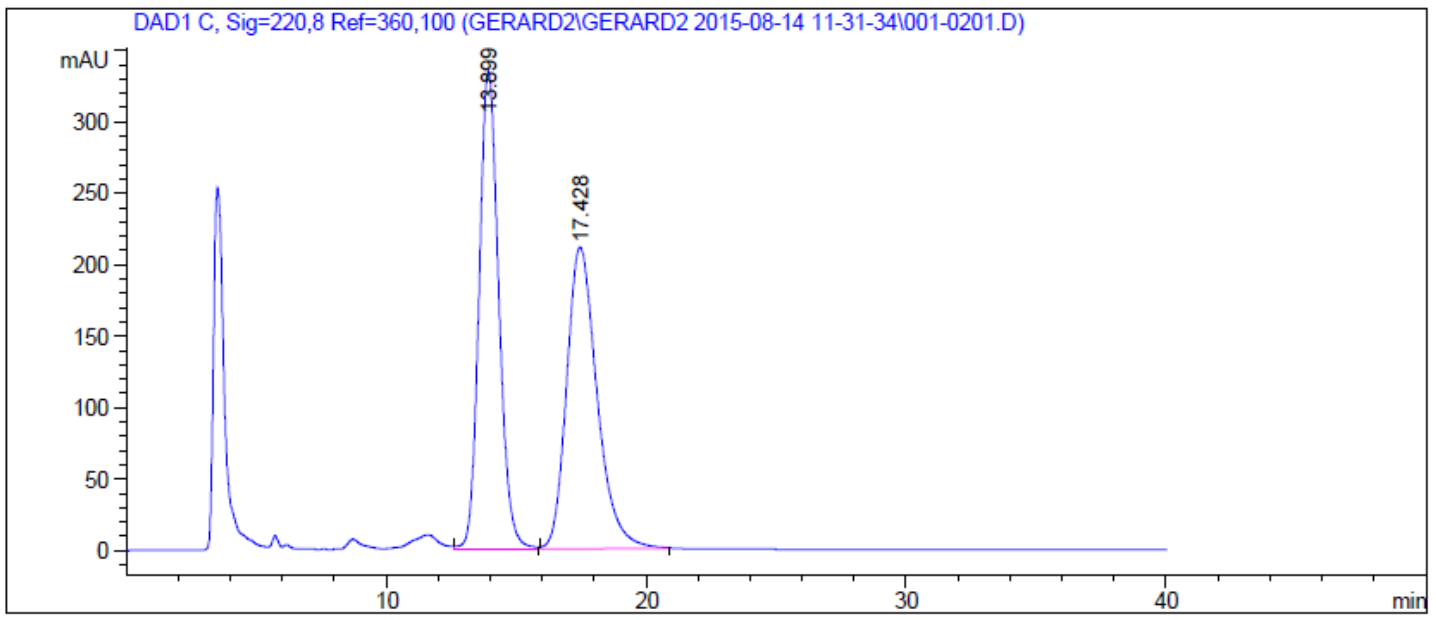

Signal 3: DAD1 C, Sig $=220,8$ Ref $=360,100$

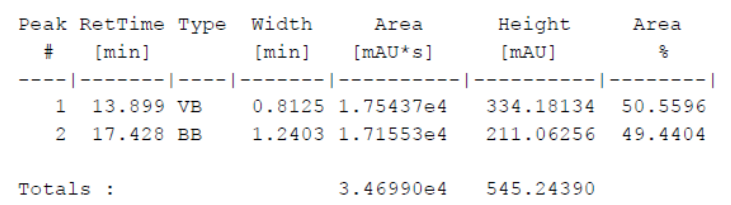




\section{HPLC chromatograms of $\mathbf{4 k}$}

Chiralpak AD-H, hexane/isopropanol = 95/5

Enantiomerically enriched (96\% ee)

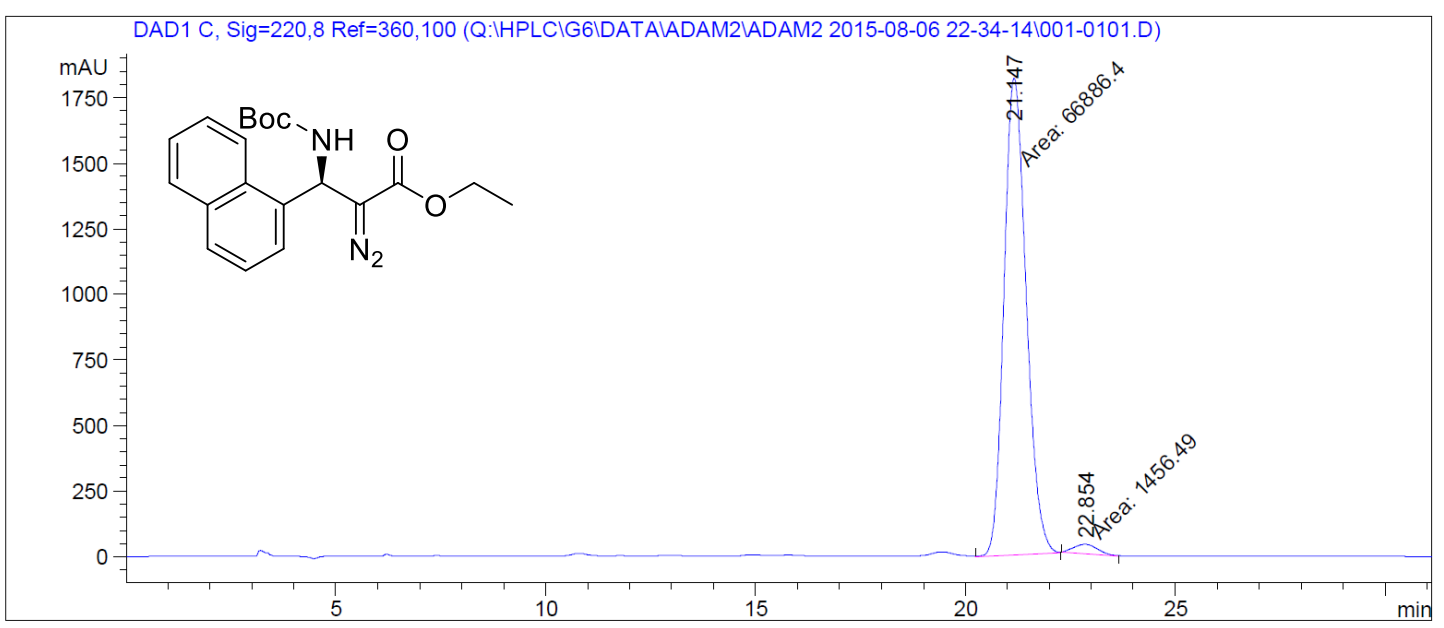

SignaL 3: DND1 C, Sig-220,8 Ret-360,100

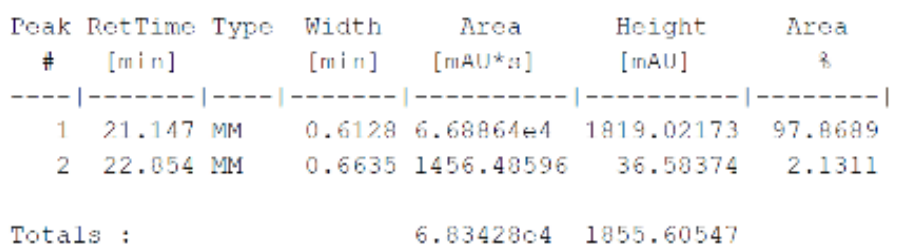

\section{Racemic}

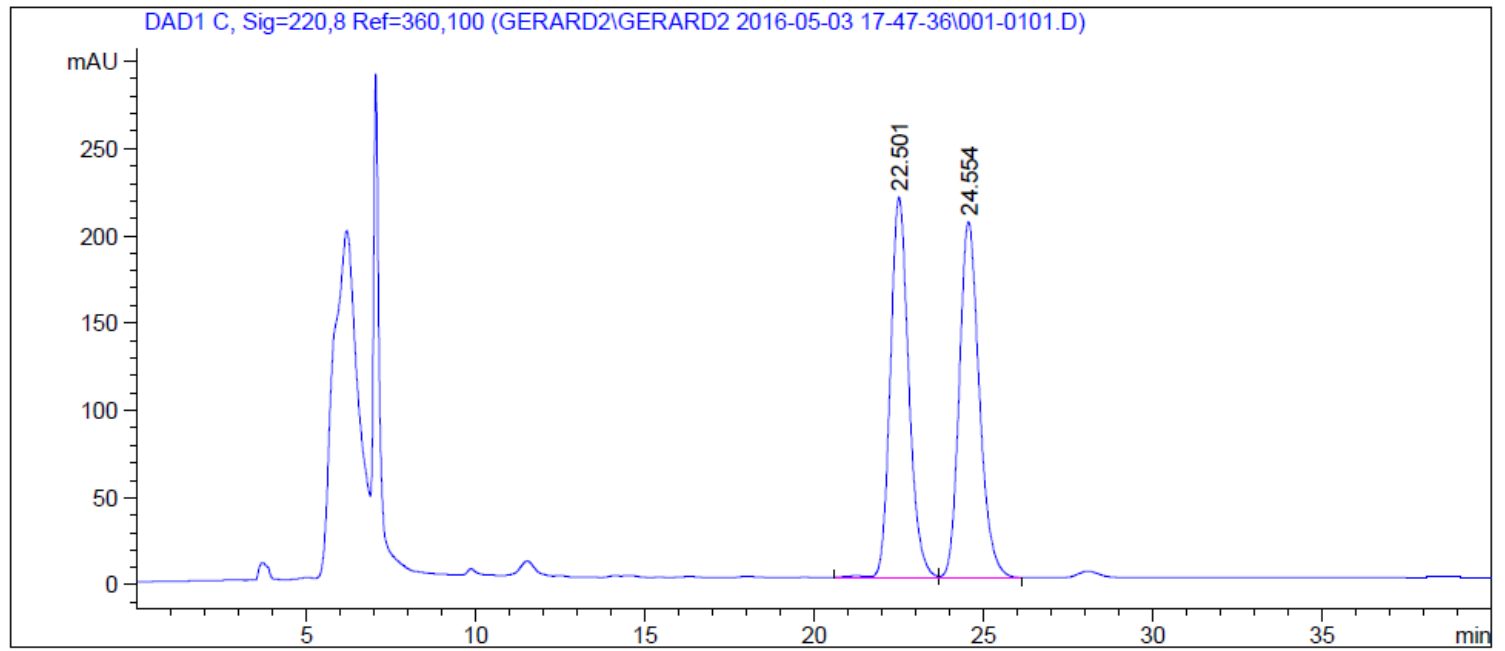

Signal 3: DAD1 C, Sig=220,8 Ref $=360,100$

\begin{tabular}{|c|c|c|c|c|c|c|}
\hline $\begin{array}{c}\text { Peak } \\
\#\end{array}$ & $\begin{array}{c}\text { RetTime } \\
\text { [min] }\end{array}$ & Type & $\begin{array}{l}\text { Width } \\
\text { [min] }\end{array}$ & $\begin{array}{c}\text { Area } \\
{\left[\mathrm{mAU}^{*} \mathrm{~S}\right]}\end{array}$ & $\begin{array}{l}\text { Height } \\
\text { [mAU] }\end{array}$ & $\begin{array}{c}\text { Area } \\
\frac{8}{8}\end{array}$ \\
\hline & 20501 & BV & -----1 & 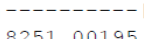 & - & ------1 \\
\hline $\begin{array}{l}1 \\
2\end{array}$ & $\begin{array}{l}22.501 \\
24.554\end{array}$ & $\begin{array}{l}\text { BV } \\
\text { VB }\end{array}$ & $\begin{array}{l}0.5816 \\
0.6317\end{array}$ & $\begin{array}{l}8251.00195 \\
8391.71582\end{array}$ & $\begin{array}{l}217.96738 \\
204.07736\end{array}$ & $\begin{array}{l}49.5773 \\
50.4227\end{array}$ \\
\hline t. & : & & & $1.66427 e 4$ & 422.04474 & \\
\hline
\end{tabular}




\section{HPLC chromatograms of 41}

Chiralpak AD-H, hexane/isopropanol = 95/5, $1 \mathrm{~mL} / \mathrm{min}$

Enantiomerically enriched ( $97 \%$ ee)

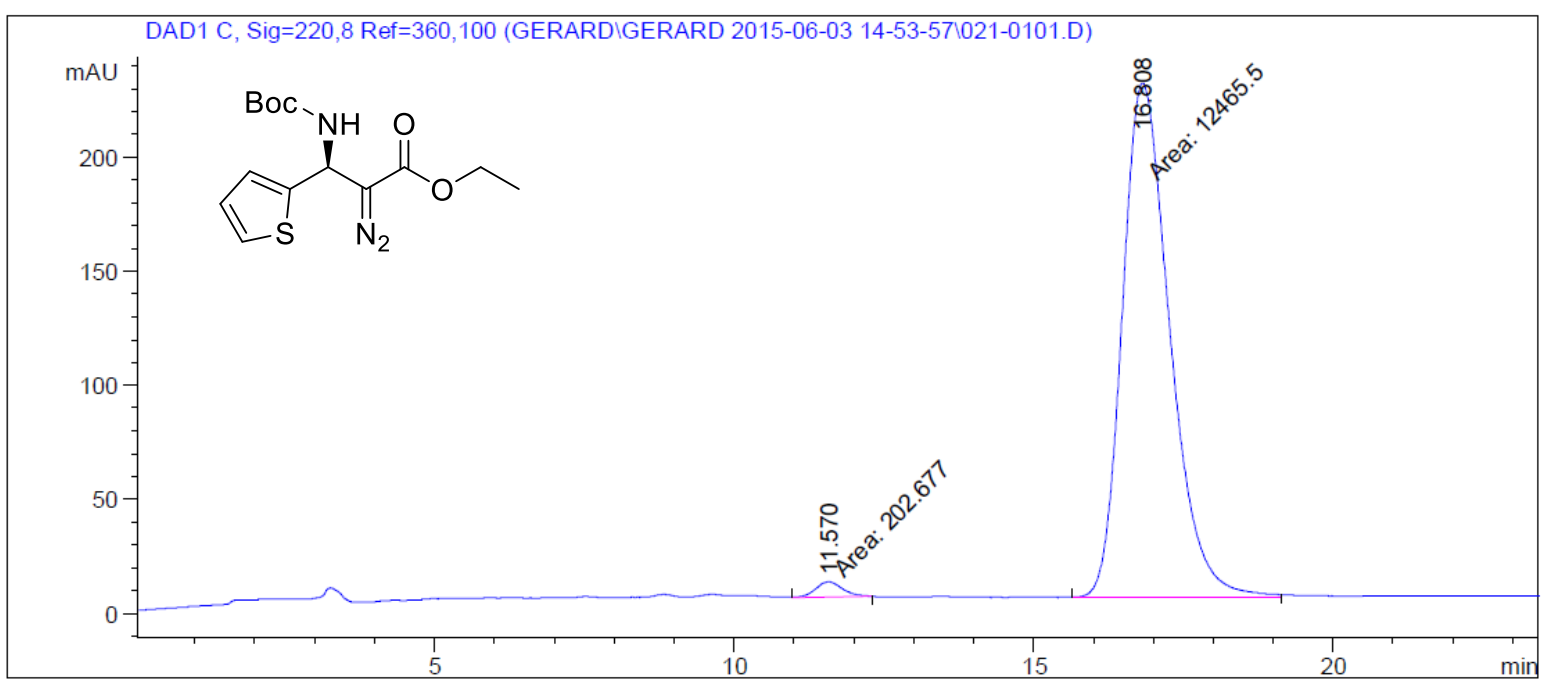

Signal 3: DAD1 C, $\operatorname{Sig}=220,8$ Ref $=360,100$

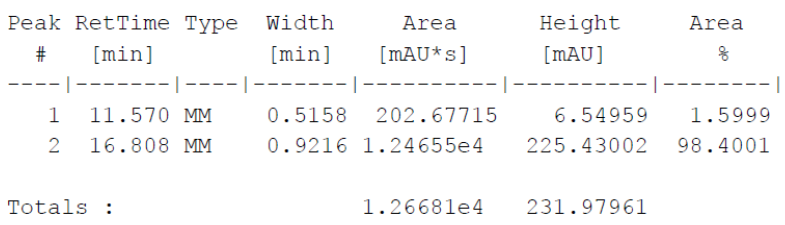

\section{Racemic}

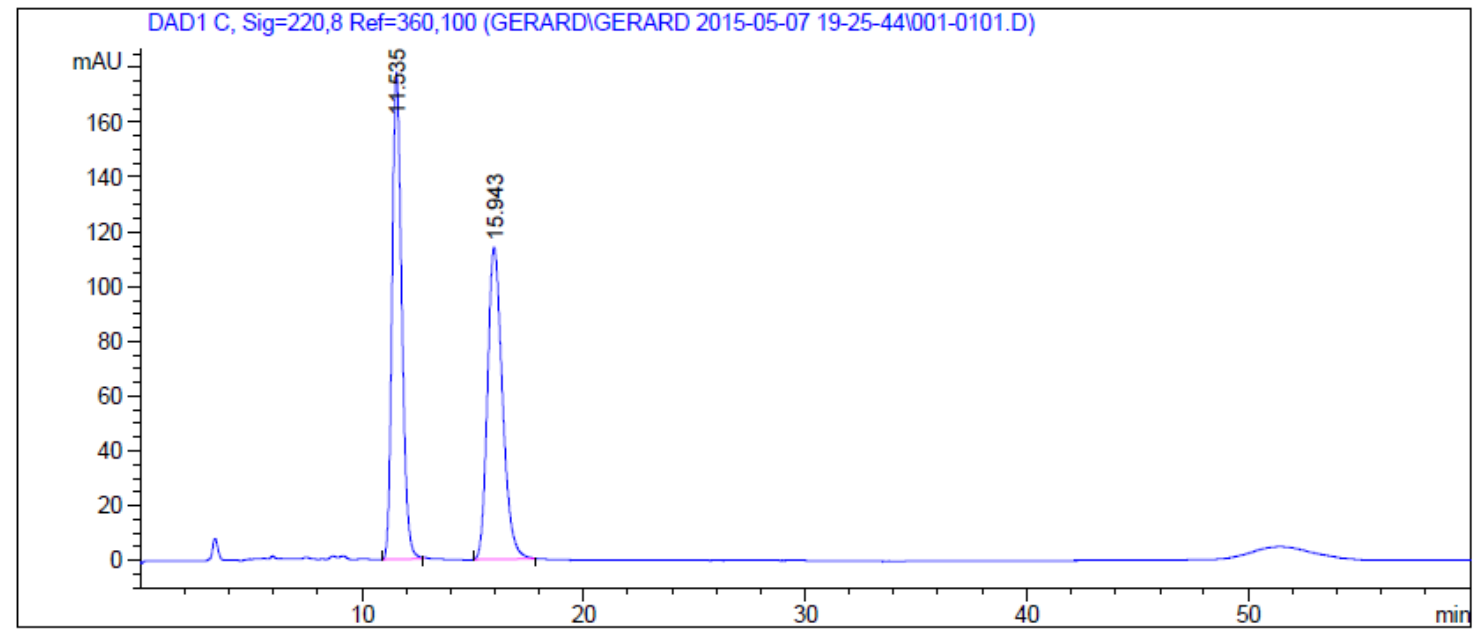

Signal 3: DAD1 C, $\operatorname{Sig}=220,8$ Ref $=360,100$

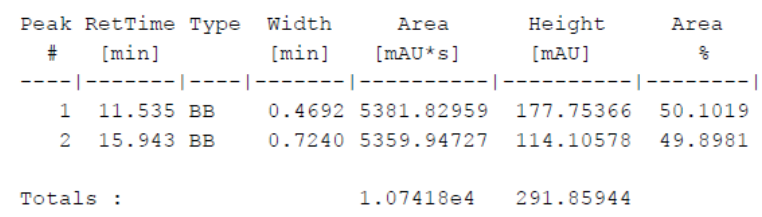




\section{HPLC chromatograms of $\mathbf{4 m}$}

Chiralpak AD-H, hexane/isopropanol = 95/5, $1 \mathrm{~mL} / \mathrm{min}$

Enantiomerically enriched ( $98 \%$ ee)
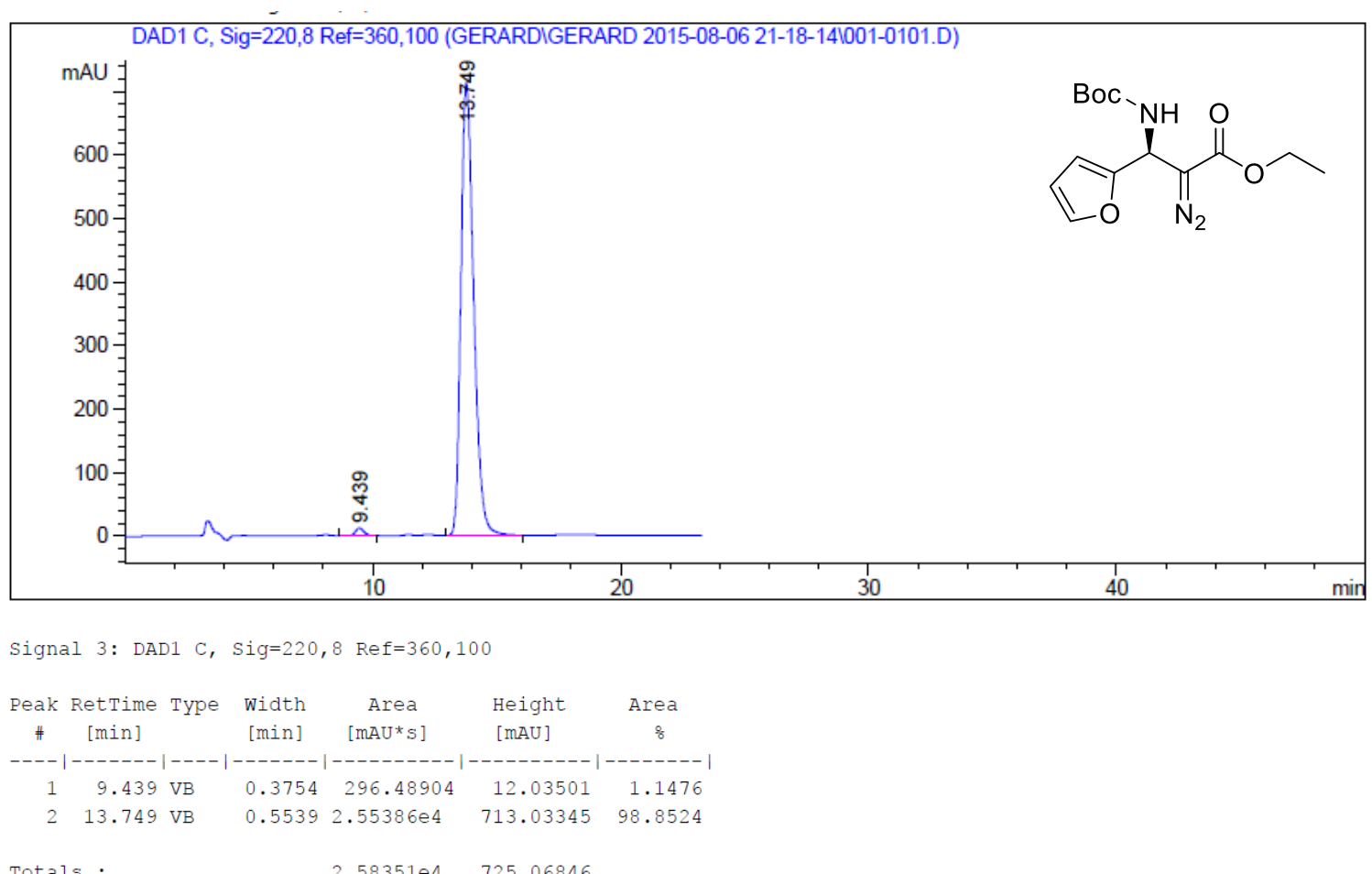

$2.58351 e 4 \quad 725.06846$

\section{Racemic}

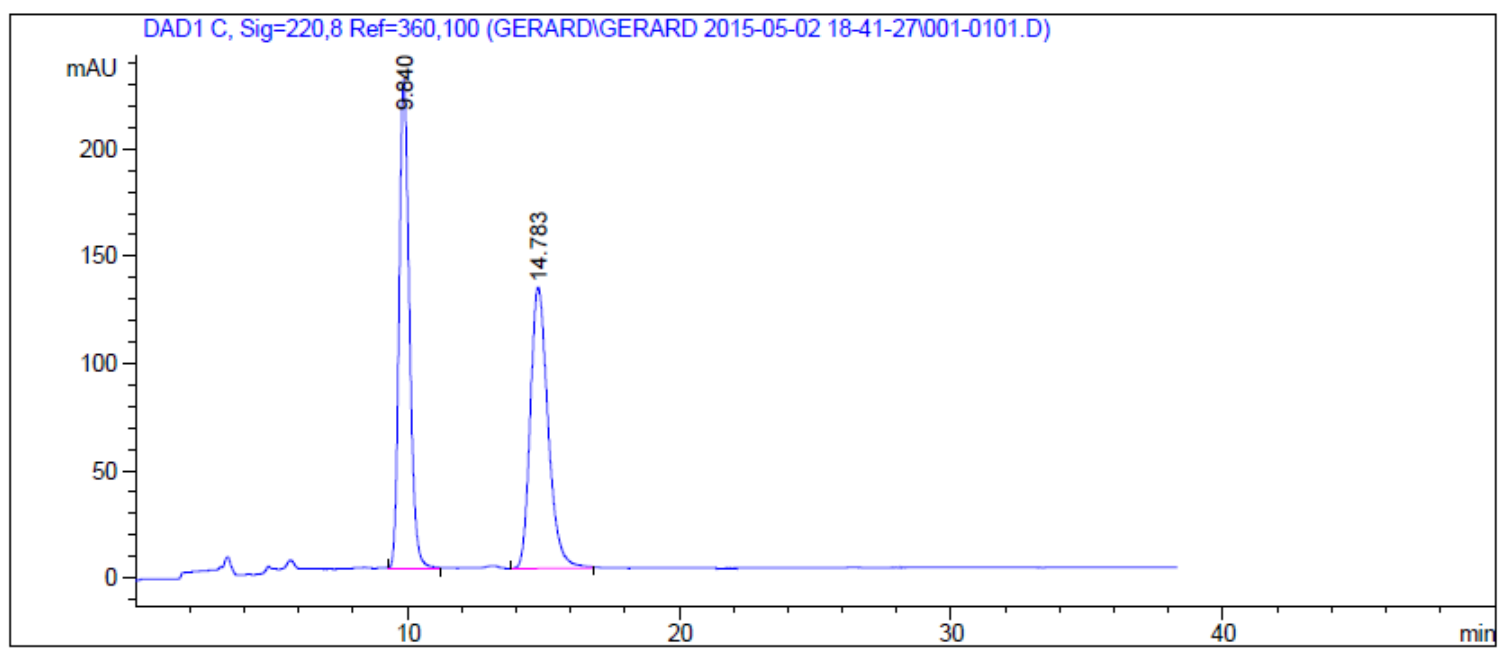

Signal 3: DAD1 C, Sig $=220,8$ Ref $=360,100$

\begin{tabular}{|c|c|c|c|c|c|c|}
\hline $\begin{array}{c}\text { Peak } \\
\quad \#\end{array}$ & $\begin{array}{c}\text { RetTime } \\
\text { [min] }\end{array}$ & Type & $\begin{array}{l}\text { width } \\
\text { [min] }\end{array}$ & $\begin{array}{c}\text { Area } \\
{\left[\mathrm{mAU}^{\star} \mathrm{s}\right]}\end{array}$ & $\begin{array}{l}\text { Height } \\
\text { [MAU] }\end{array}$ & $\begin{array}{c}\text { Area } \\
\frac{8}{6}\end{array}$ \\
\hline & -1 & 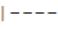 & -- & |----_---- & 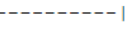 & -------1 \\
\hline 1 & 9.840 & BB & 0.4049 & 5986.87158 & 227.55272 & 50.2055 \\
\hline 2 & 14.783 & VB & 0.6928 & 5937.86230 & 131.01543 & 49.7945 \\
\hline & 5 & & & $1.19247 e 4$ & 358.56815 & \\
\hline
\end{tabular}




\section{HPLC chromatograms of $\mathbf{4 n}$}

Chiralpak AD-H, hexane/isopropanol = 95/5, $1 \mathrm{~mL} / \mathrm{min}$

Enantiomerically enriched ( $92 \%$ ee)

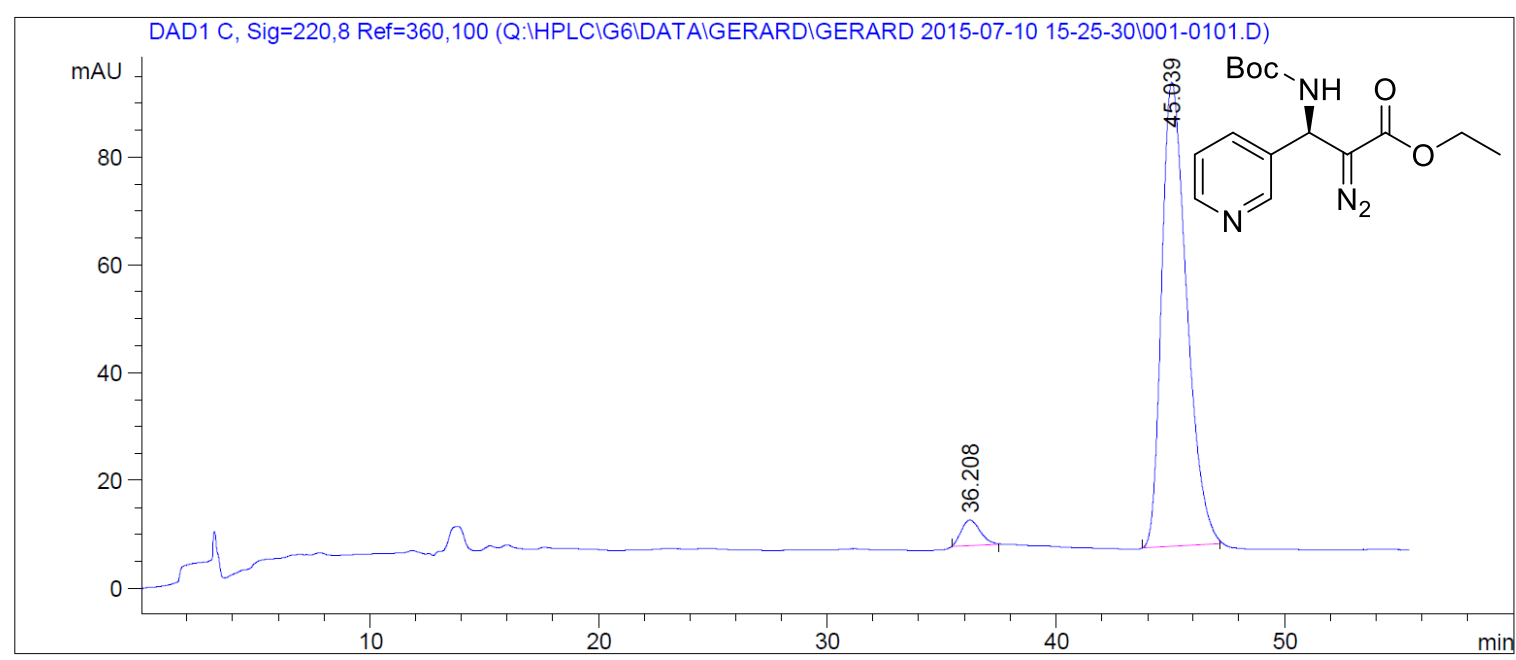

Signal 3: DAD1 C, Sig=220,8 Ref $=360,100$

\begin{tabular}{|c|c|c|c|c|c|c|}
\hline $\begin{array}{c}\text { Peak } \\
\#\end{array}$ & $\begin{array}{l}\text { RetTime } \\
\text { [min] }\end{array}$ & Type & $\begin{array}{l}\text { Width } \\
\text { [min] }\end{array}$ & $\begin{array}{c}\text { Area } \\
{\left[\mathrm{mAU}{ }^{\star} \mathrm{s}\right]}\end{array}$ & $\begin{array}{l}\text { Height } \\
\text { [MAU] }\end{array}$ & $\begin{array}{c}\text { Area } \\
8\end{array}$ \\
\hline-1 & - & & $m$ & 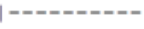 & 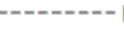 & ------ \\
\hline 1 & 36.208 & BB & 0.7167 & 275.57565 & 4.79340 & 4.0245 \\
\hline 2 & 45.039 & BB & 1.1620 & 6571.92627 & 86.14942 & 95.9755 \\
\hline
\end{tabular}

\section{Racemic}

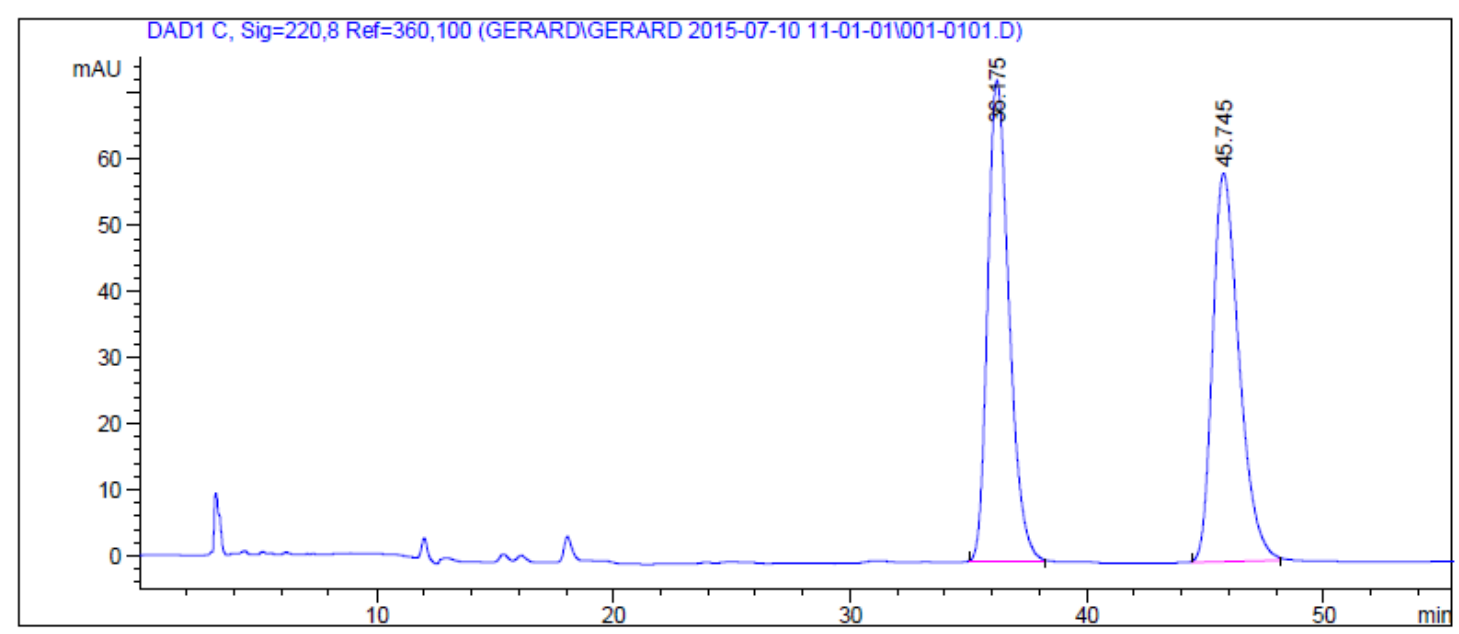

Signal 3: DAD1 C, Sig $=220,8$ Ref $=360,100$

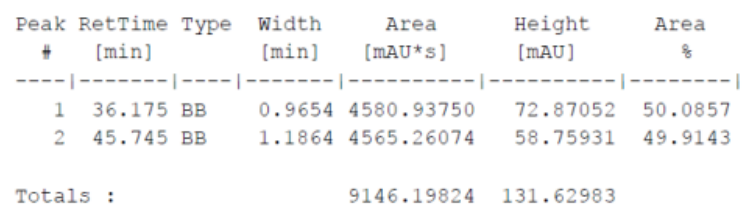


HPLC chromatograms of 40

Chiralpak AS-H, hexane/isopropanol = 90/10, $1 \mathrm{~mL} / \mathrm{min}$

Enantiomerically enriched (94\% ee)

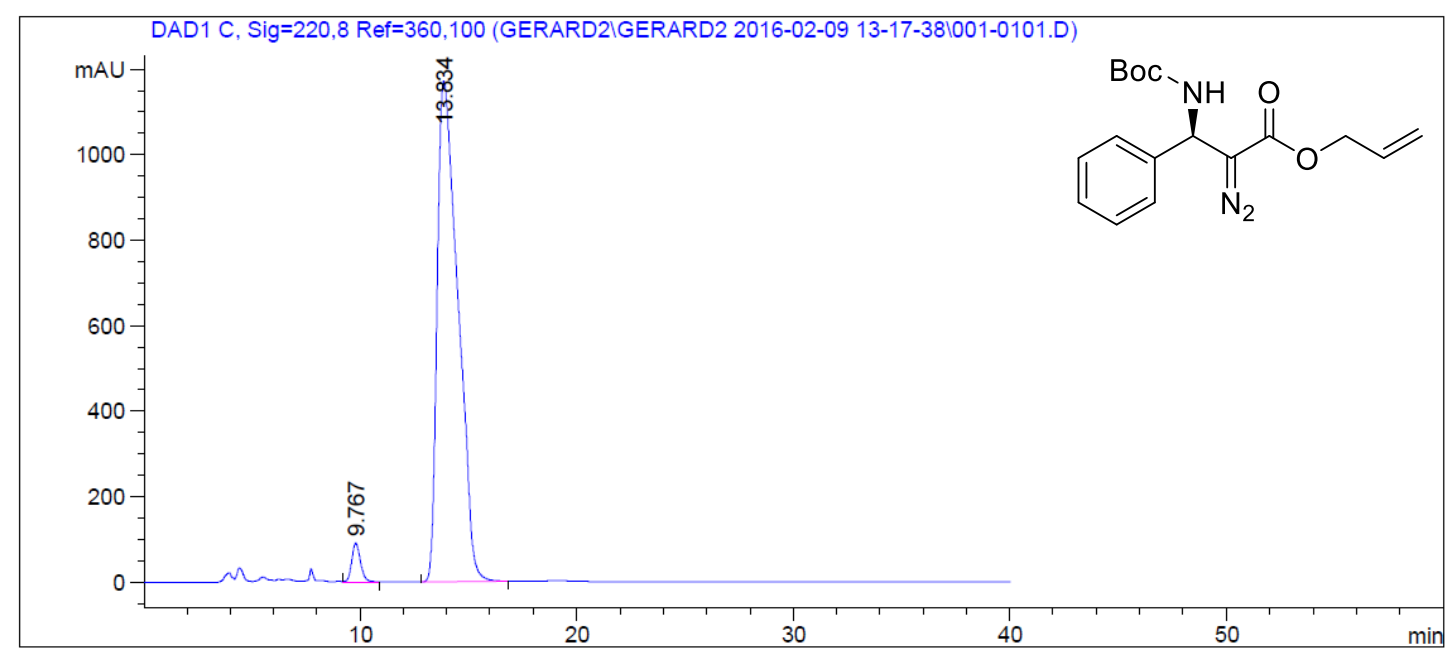

Signal 3: DAD1 C, $\operatorname{Sig}=220,8$ Ref $=360,100$

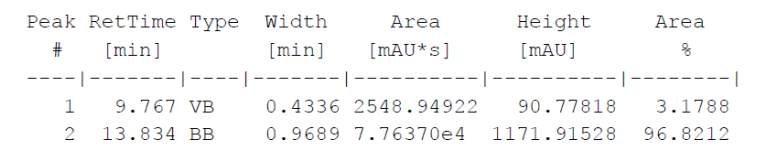

\section{Racemic}

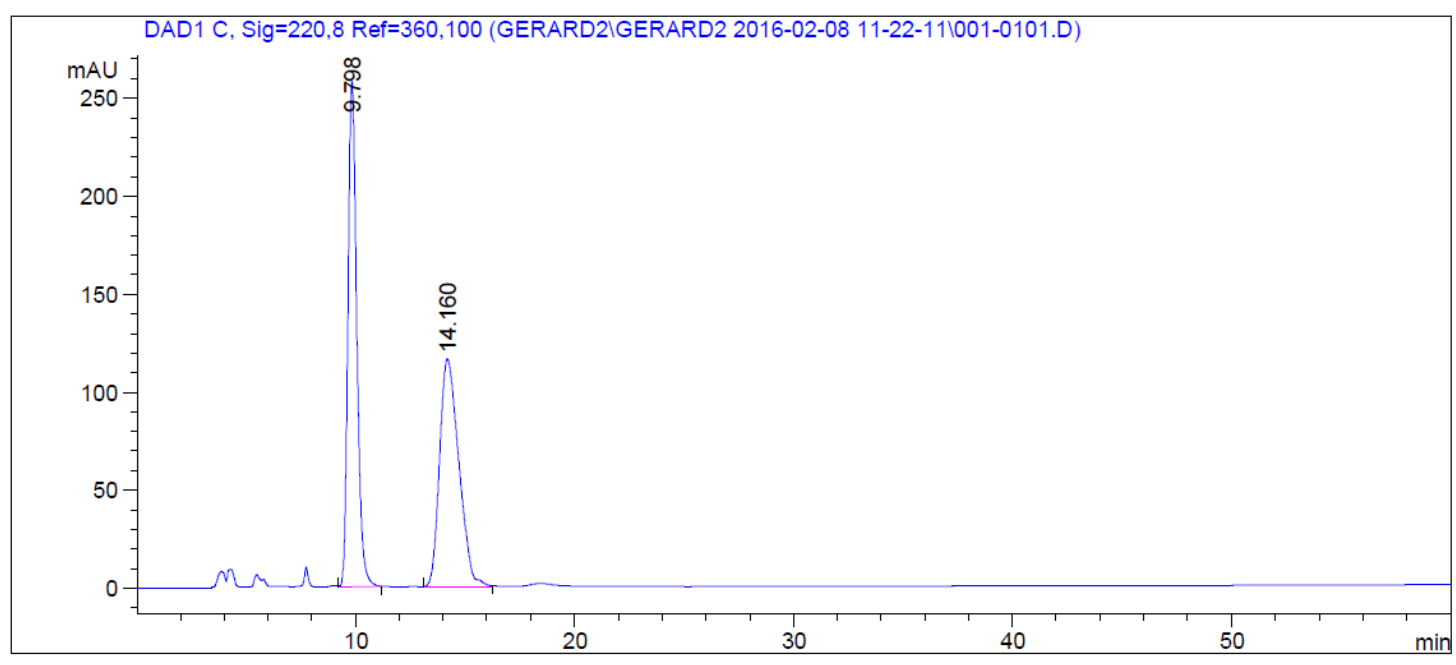

\footnotetext{
Signal 3: DAD1 C, $\operatorname{Sig}=220,8$ Ref $=360,100$

\begin{tabular}{|c|c|c|c|c|c|c|}
\hline $\begin{array}{c}\text { Peak } \\
\quad \#\end{array}$ & $\begin{array}{l}\text { RetTime } \\
\text { [min] }\end{array}$ & Type & $\begin{array}{l}\text { Width } \\
\text { [min] }\end{array}$ & $\begin{array}{c}\text { Area } \\
{\left[\mathrm{mAU}{ }^{*} \mathrm{~s}\right]}\end{array}$ & $\begin{array}{l}\text { Height } \\
\text { [mAU] }\end{array}$ & $\begin{array}{c}\text { Area } \\
\&\end{array}$ \\
\hline 1 & 9.798 & $\mathrm{VB}$ & 0.4301 & 7215.45605 & 258.17068 & 50.4871 \\
\hline 2 & 14.160 & $\mathrm{BB}$ & 0.9429 & 7076.23340 & 116.12497 & 49.5129 \\
\hline
\end{tabular}
}


HPLC chromatograms of $\mathbf{4 p}$

Chiralpak IA, hexane/isopropanol = 95/5, $1 \mathrm{~mL} / \mathrm{min}$

Enantiomerically enriched $92 \%$ ee

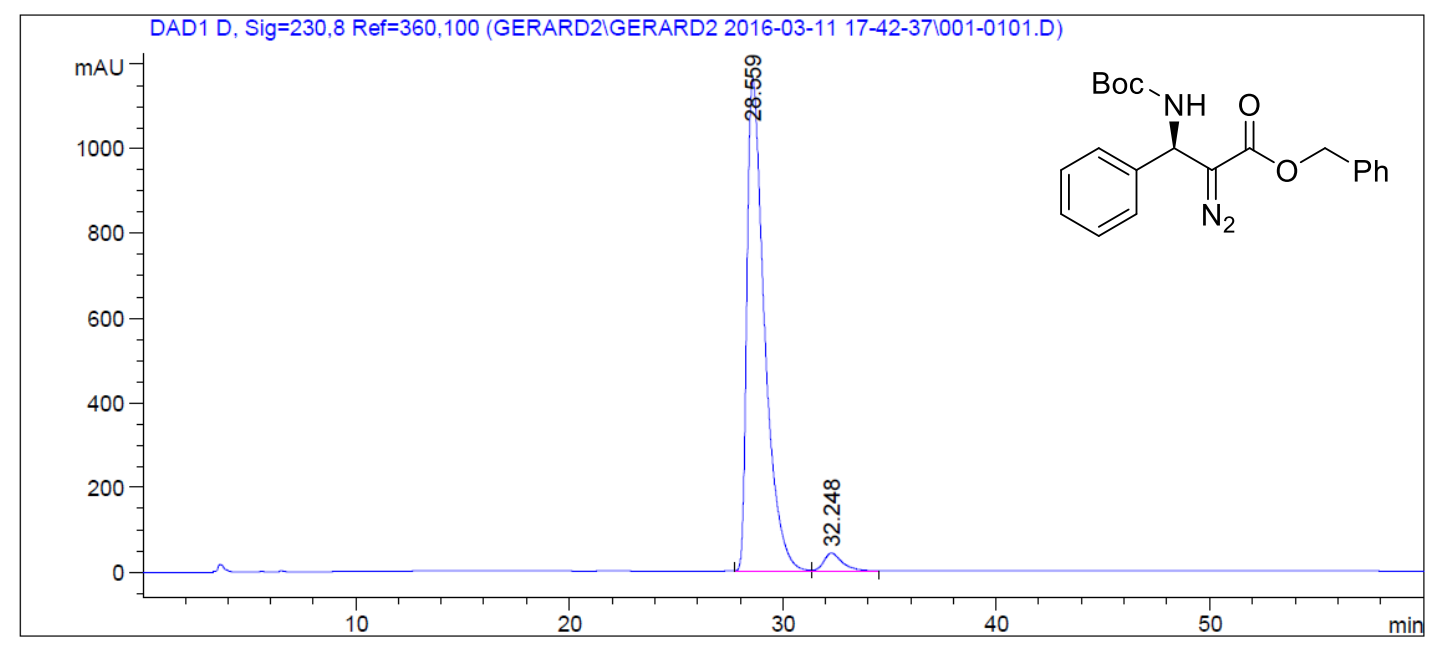

Signal 3: DAD1 C, Sig $=220,8$ Ref $=360,100$

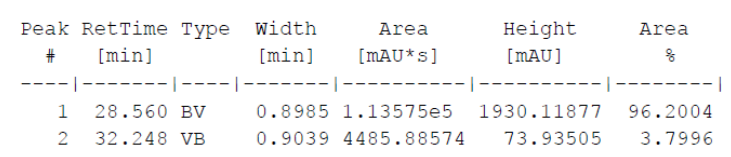

Racemic

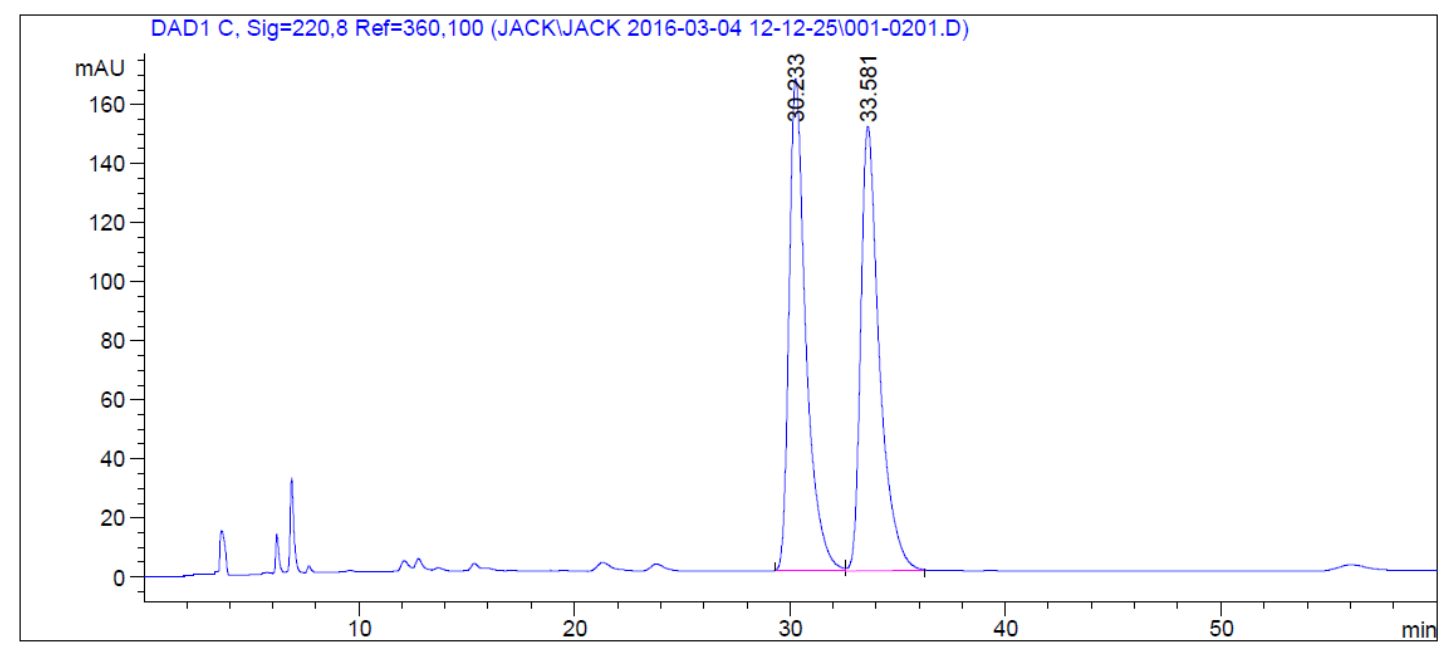

\footnotetext{
Signal 3: DAD1 C, Sig $=220,8$ Ref $=360,100$

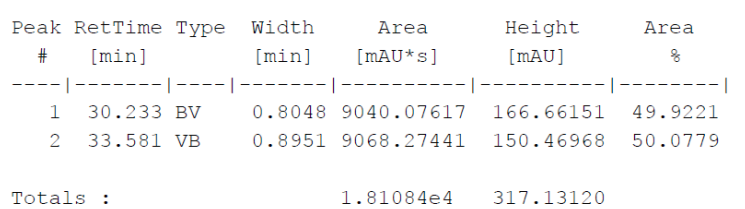


HPLC chromatograms of $\mathbf{4 q}$

Chiralpak AS-H, hexane/isopropanol = 95/5, $1 \mathrm{~mL} / \mathrm{min}$

Enantiomerically enriched (88\% ee)

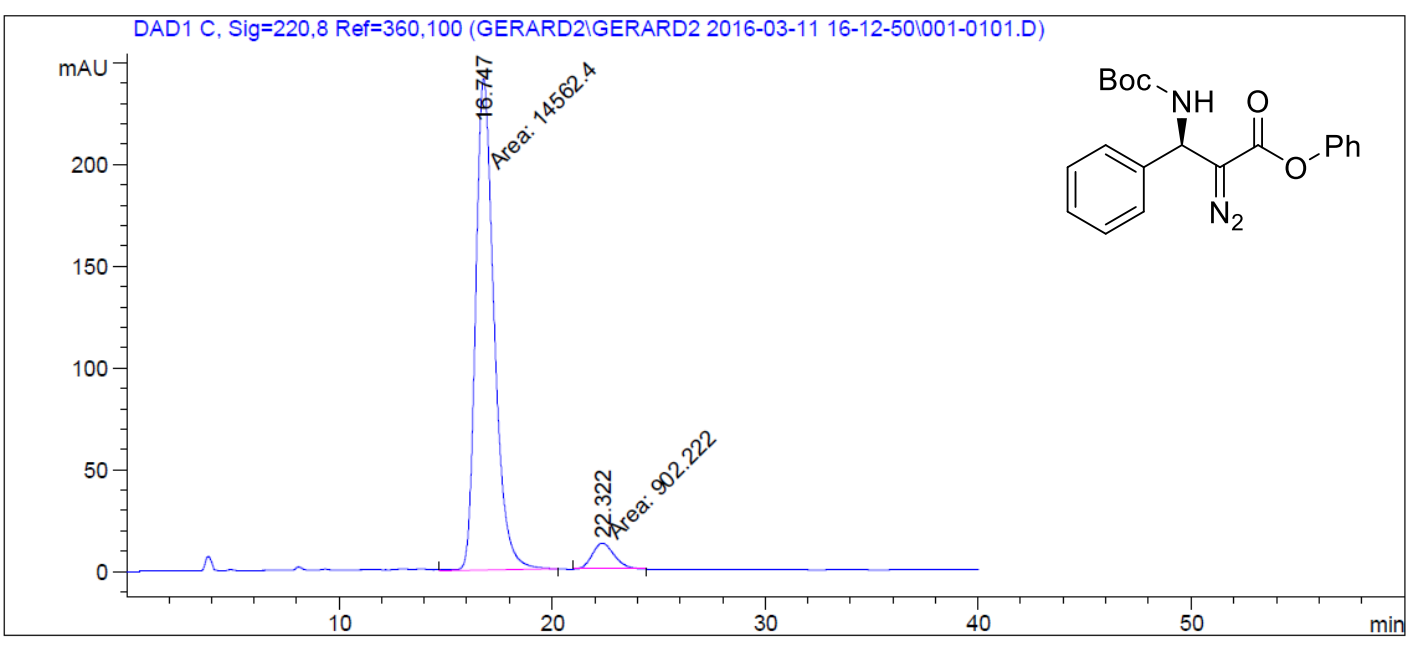

Signal 3: DAD1 C, Sig=220, 8 Ref $=360,100$

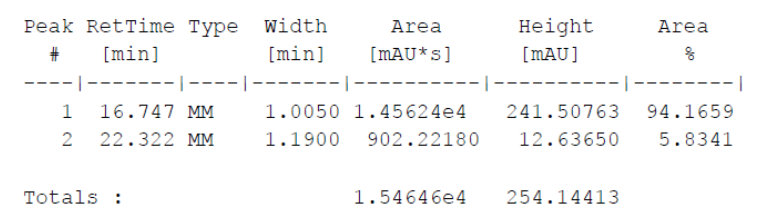

\section{Racemic}

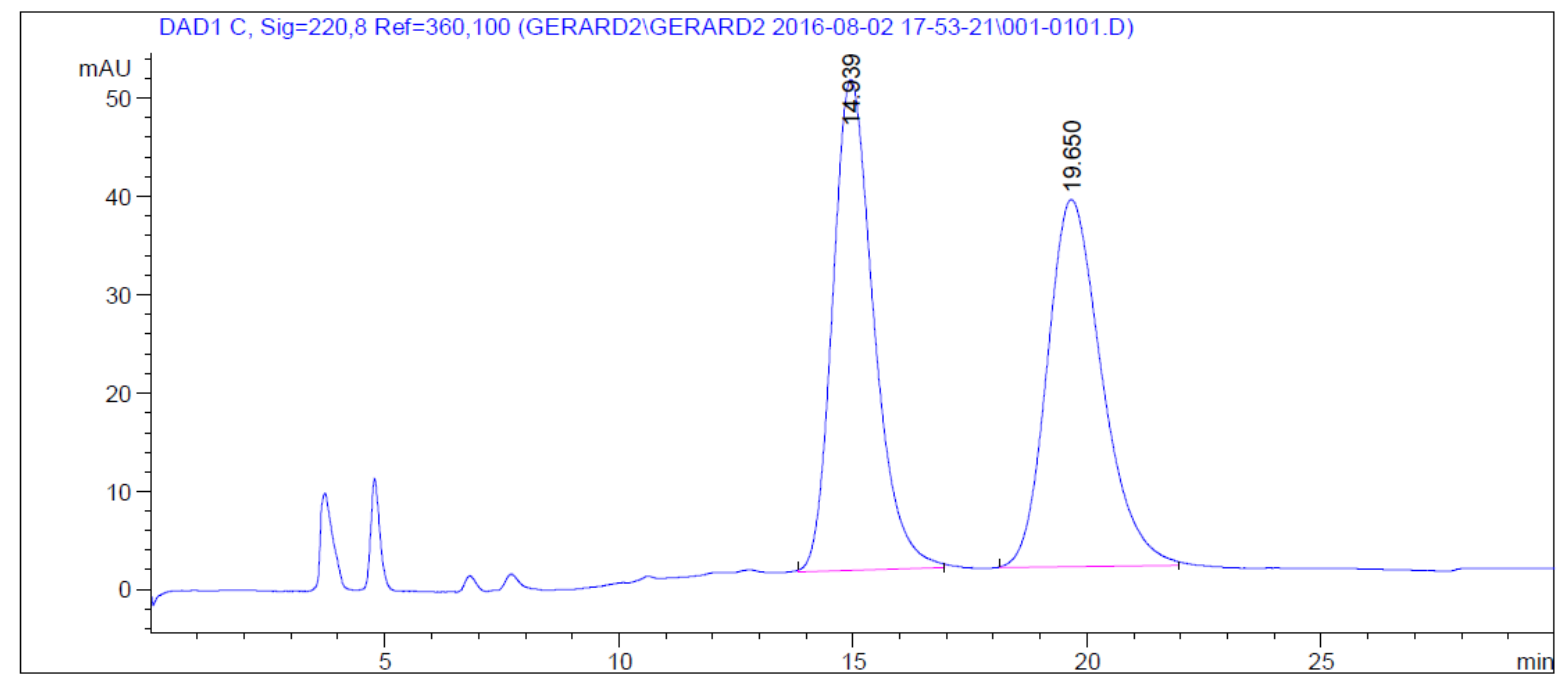

Signal 3: DAD1 C, $\operatorname{Sig}=220,8 \operatorname{Ref}=360,100$

\begin{tabular}{|c|c|c|c|c|c|c|}
\hline $\begin{array}{c}\text { Peak } \\
\quad \#\end{array}$ & $\begin{array}{c}\text { RetTime } \\
\text { [min] }\end{array}$ & Type & $\begin{array}{l}\text { Width } \\
\text { [min] }\end{array}$ & $\begin{array}{c}\text { Area } \\
{[\mathrm{mAU} * \mathrm{~s}]}\end{array}$ & $\begin{array}{l}\text { Height } \\
\text { [mAU] }\end{array}$ & $\begin{array}{c}\text { Area } \\
\&\end{array}$ \\
\hline---- & |------ & 7 & $-------\mid$ & |---------- | & $---------\mid$ & $--------\mid$ \\
\hline 1 & 14.939 & $\mathrm{BB}$ & 0.9179 & 3036.14209 & 49.89961 & 50.0900 \\
\hline 2 & 19.650 & $\mathrm{BB}$ & 1.2335 & 3025.23730 & 37.32967 & 49.9100 \\
\hline ota. & Is : & & & 6061.37939 & 87.22927 & \\
\hline
\end{tabular}




\section{HPLC chromatograms of $\mathbf{5 a}$}

Chiralpak OD, hexane/isopropanol = 98/2,1 $\mathrm{mL} / \mathrm{min}$

\section{Enantioenriched 96\% ee}

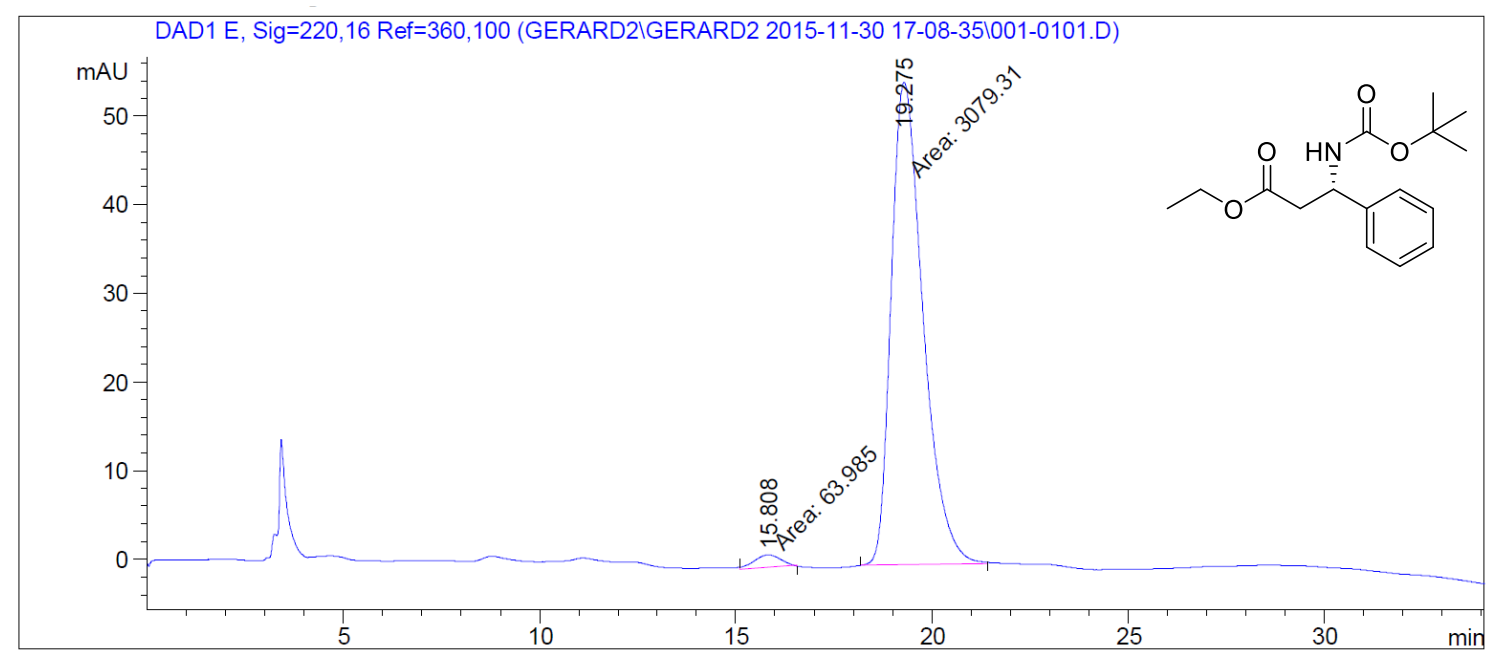

Signal 5: DAD1 E, $\operatorname{Sig}=220,16$ Ref $=360,100$

\begin{tabular}{|c|c|c|c|c|c|c|}
\hline $\begin{array}{c}\text { eak } \\
\#\end{array}$ & $\begin{array}{l}\text { RetTime } \\
\text { [min] }\end{array}$ & Type & $\begin{array}{l}\text { width } \\
\text { [min] }\end{array}$ & $\begin{array}{c}\text { Area } \\
{\left[\mathrm{mAU}^{\star} \mathrm{s}\right]}\end{array}$ & $\begin{array}{l}\text { Height } \\
\text { [mAU] }\end{array}$ & $\begin{array}{c}\text { Area } \\
\frac{8}{8}\end{array}$ \\
\hline 1 & & & 0.7709 & 5 & 1 & 56 \\
\hline 2 & 19.275 & $\mathrm{MM}$ & 0.9426 & 3079.30518 & 54.44812 & 97.9644 \\
\hline
\end{tabular}

\section{Racemic}

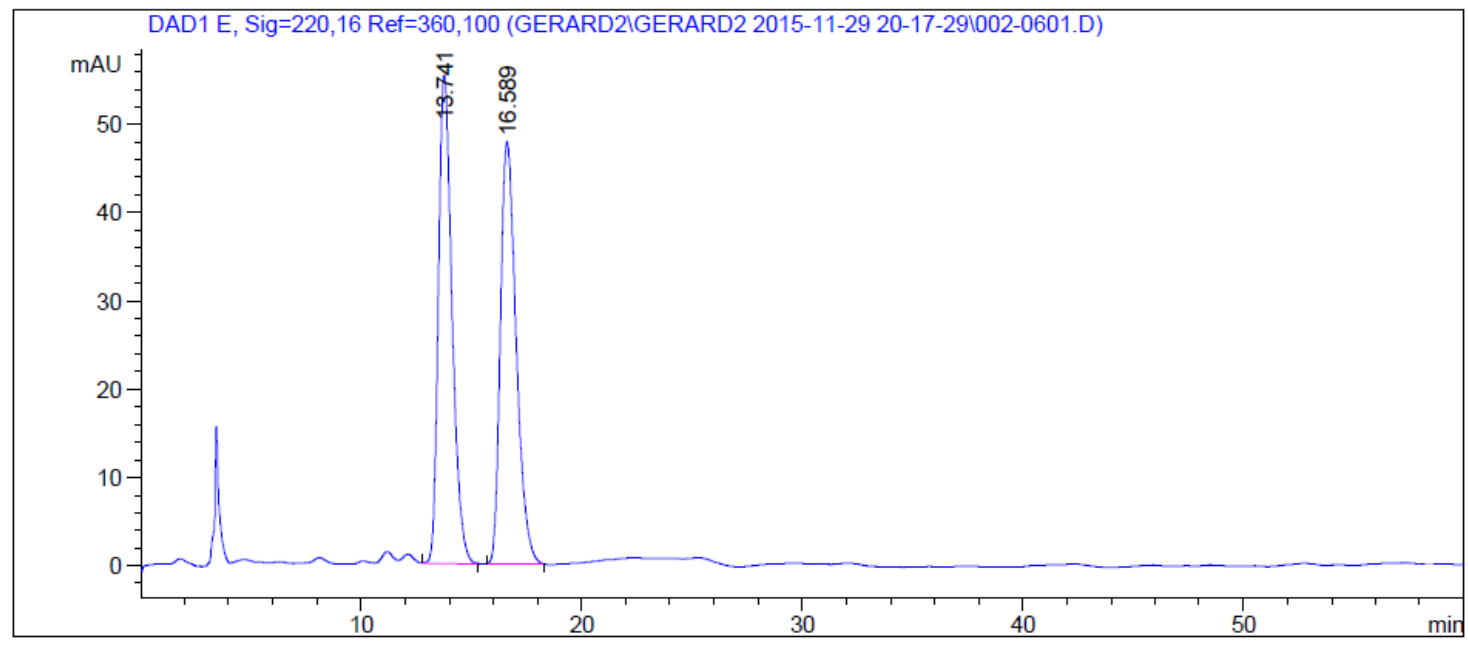

Signal 5: DAD1 E, Sig $=220,16$ Ref $=360,100$

\begin{tabular}{|c|c|c|c|c|c|c|}
\hline $\begin{array}{c}\text { Peak } \\
4\end{array}$ & $\begin{array}{c}\text { RetTime } \\
\text { [min] }\end{array}$ & Type & $\begin{array}{l}\text { Width } \\
\text { [min] }\end{array}$ & $\begin{array}{c}\text { Area } \\
{\left[\mathrm{mAU}{ }^{*} \mathrm{~S}\right]}\end{array}$ & $\begin{array}{l}\text { Height } \\
\text { [mAU] }\end{array}$ & $\begin{array}{c}\text { Area } \\
\&\end{array}$ \\
\hline 1 & 13,741 & $\mathrm{VB}_{\mathrm{B}}$ & 0.6611 & 2378.39795 & 55.37799 & 50.0068 \\
\hline 2 & 16.589 & $\mathrm{BB}$ & 0.7717 & 2377.74805 & 47.85278 & 49.9932 \\
\hline Tota & & & & 4756.14600 & 103.23077 & \\
\hline
\end{tabular}




\section{HPLC chromatograms of 5c}

Chiralpak AD-H, hexane/isopropanol = 80/20, $1 \mathrm{~mL} / \mathrm{min}$

\section{Enantiomerically enriched ( $96 \%$ ee)}
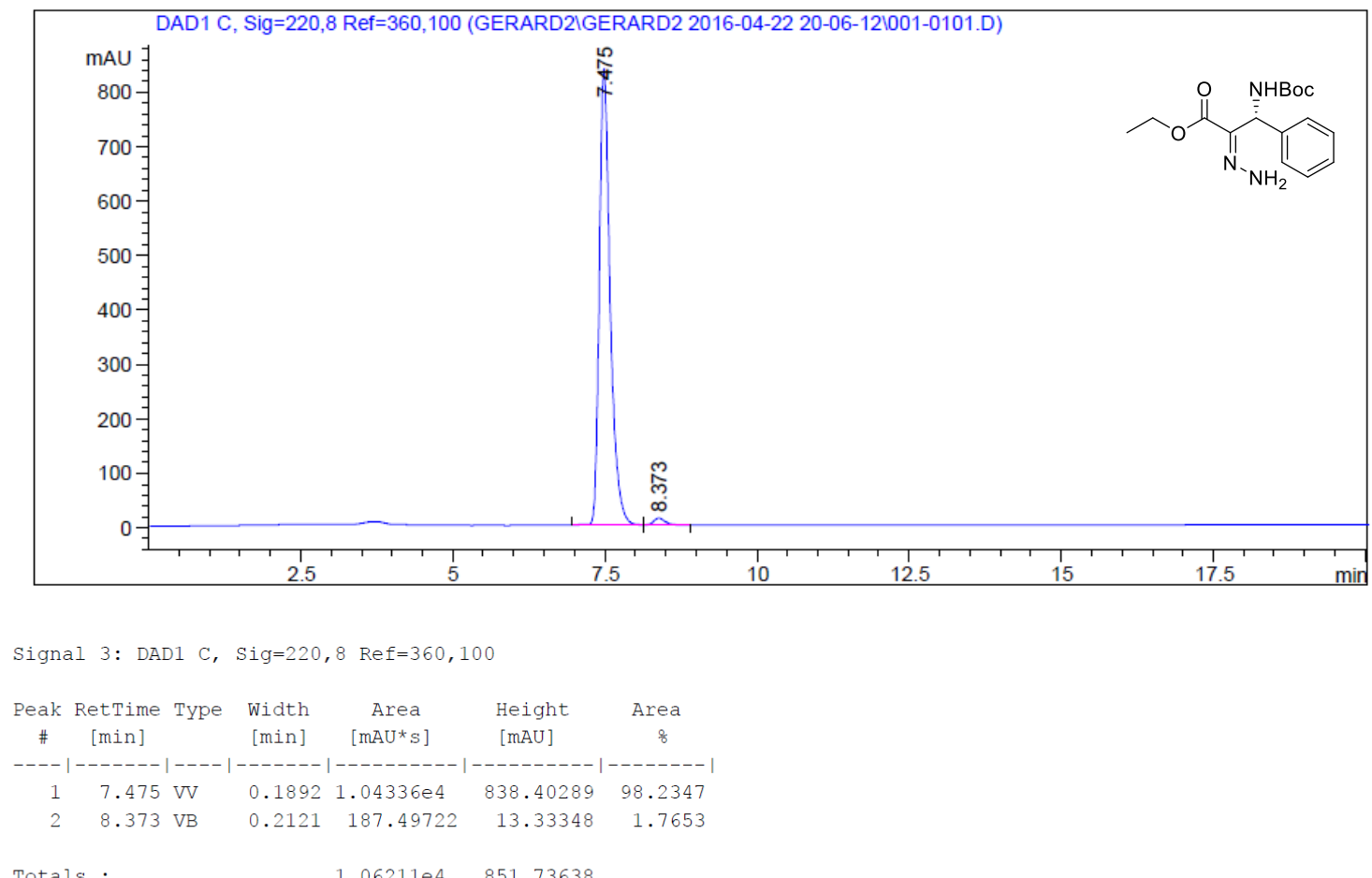

\section{Racemic}

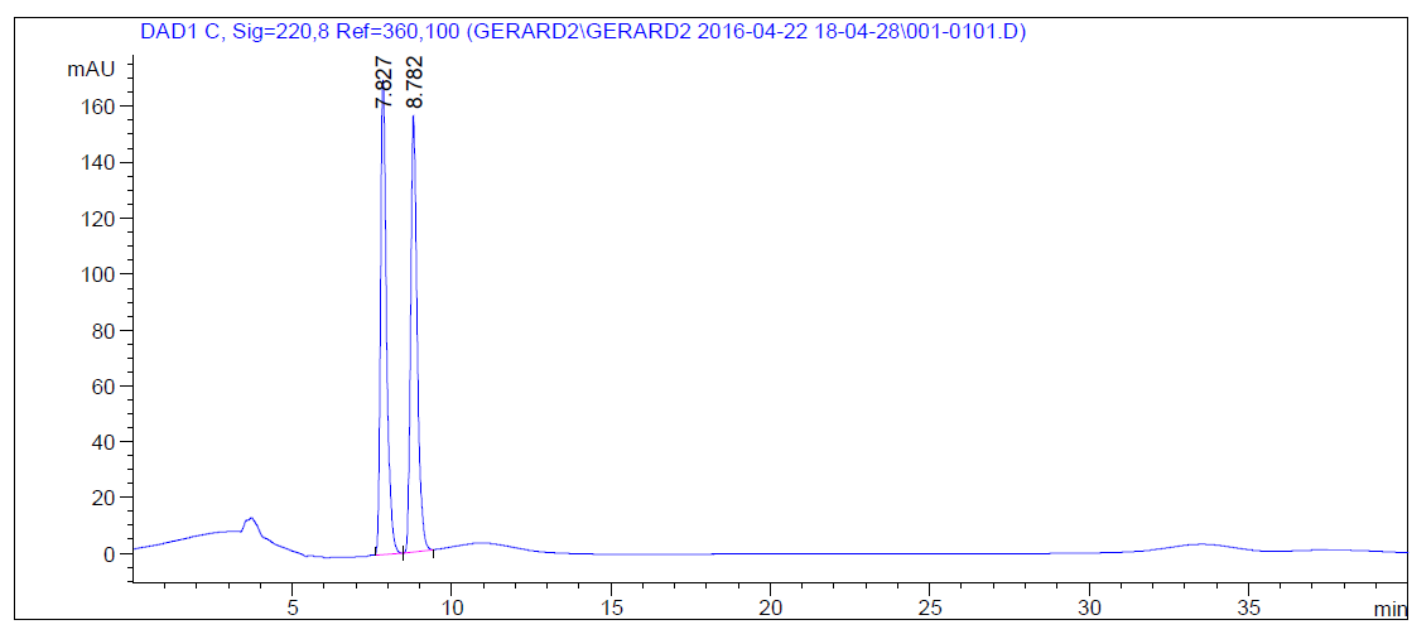

Signal 3: DAD1 C, Sig $=220,8$ Ref $=360,100$

\begin{tabular}{|c|c|c|c|c|c|c|}
\hline $\begin{array}{c}\text { Peak } \\
\quad \#\end{array}$ & $\begin{array}{c}\text { RetTime } \\
\text { [min] }\end{array}$ & Type & $\begin{array}{l}\text { Width } \\
\text { [min] }\end{array}$ & $\begin{array}{c}\text { Area } \\
{\left[\mathrm{mAU}^{\star} \mathrm{s}\right]}\end{array}$ & $\begin{array}{l}\text { Height } \\
\text { [mAU] }\end{array}$ & $\begin{array}{c}\text { Area } \\
\%\end{array}$ \\
\hline 1 & 7.827 & $\mathrm{BV}$ & 0.2015 & 2233.60474 & 169.75914 & 50.0645 \\
\hline 2 & 8.782 & VB & 0.2184 & 2227.84814 & 156.18195 & 49.9355 \\
\hline Total & 1s : & & & 4461.45288 & 325.94109 & \\
\hline
\end{tabular}


HPLC chromatograms of $\mathbf{5 d}$

Chiralpak IA, hexane/isopropanol = 95/5, $1 \mathrm{~mL} / \mathrm{min}$

Enantiomerically enriched (96\% ee)

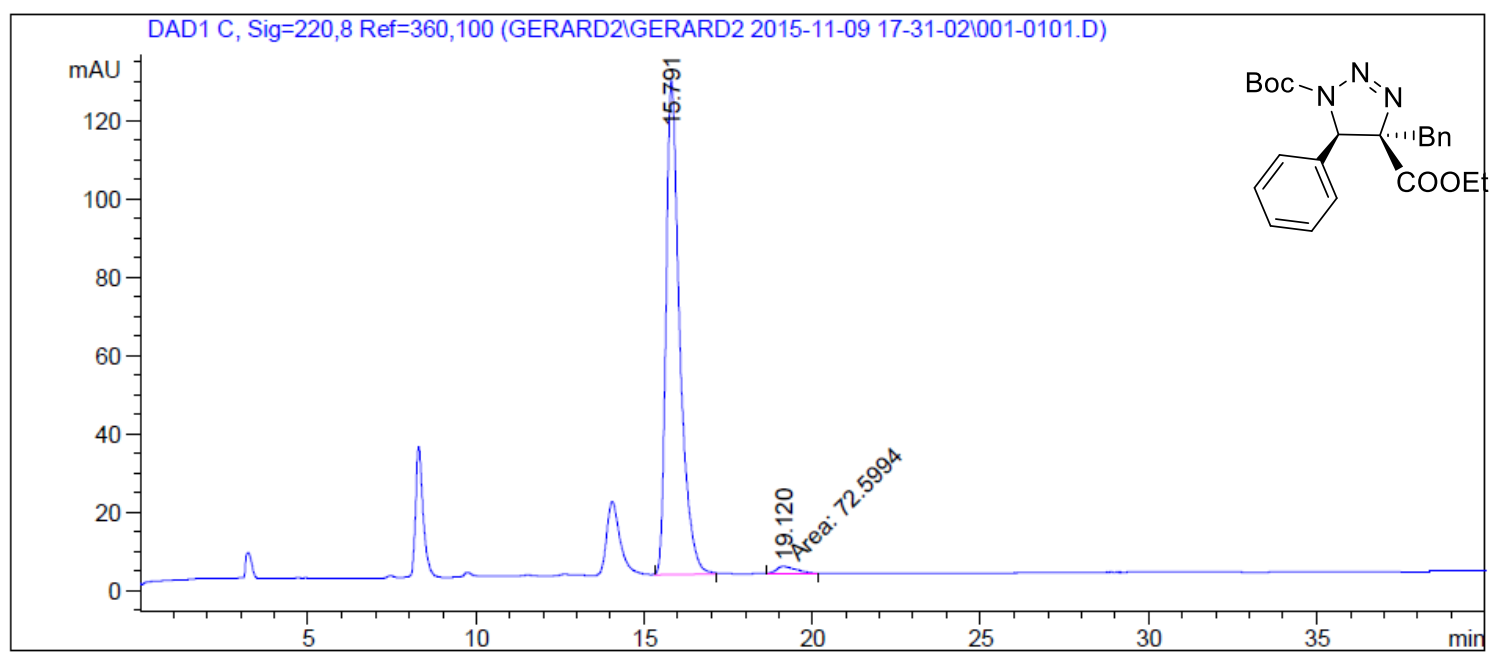

Signal 3: DAD1 C, $\operatorname{Sig}=220,8$ Ref $=360,100$

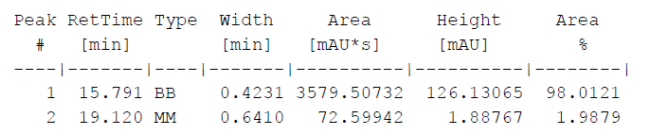

\section{Racemic}

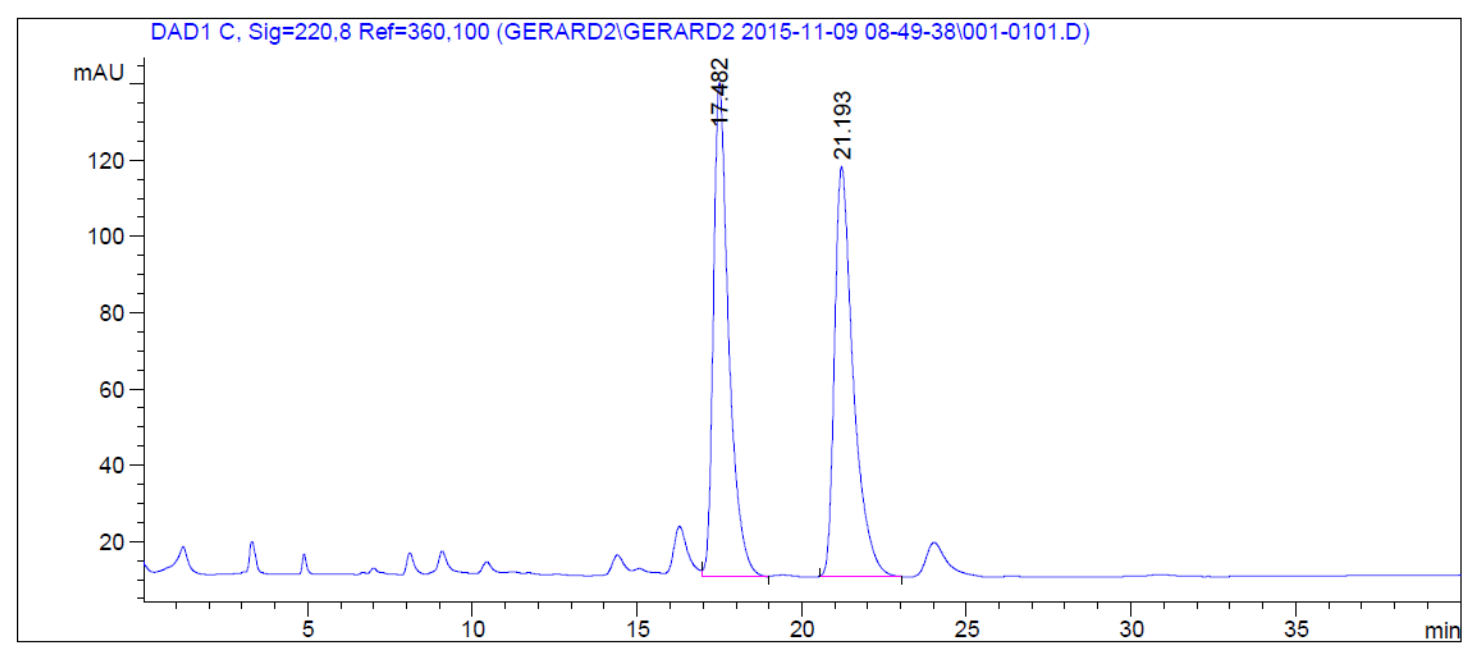

Signal 3: DAD1 C, Sig $=220,8$ Ref $=360,100$

\begin{tabular}{|c|c|c|c|c|c|c|}
\hline eak & $\begin{array}{l}\text { RetTime } \\
\text { [min] }\end{array}$ & Type & $\begin{array}{l}\text { Width } \\
\text { [min] }\end{array}$ & $\begin{array}{c}\text { Area } \\
{\left[\mathrm{mAU}{ }^{\star} \mathrm{s}\right]}\end{array}$ & $\begin{array}{l}\text { Height } \\
\text { [mAU] }\end{array}$ & $\begin{array}{c}\text { Area } \\
\frac{8}{6}\end{array}$ \\
\hline 1 & & VB & & 6 & 53 & 591 \\
\hline 2 & 21.193 & BB & 0.5909 & 4267.95117 & 107.56229 & 50.4409 \\
\hline
\end{tabular}

